\title{
UMA COMBINAÇÃO MEC/MEF PARA ANÁLISE DA INTERAÇÃO DE ESTACAS \\ INCLINADAS E O SOLO
}

Sergio Takeo Oshima

Dissertação apresentada à Escola de Engenharia da São Carlos, da Universidade de São Paulo, como parte dos requisitos para obtenção do título de Mestre em Engenharia de Estruturas

Orientador: Prof. Dr. João Batista de Paiva

São Carlos 
Aos meus pais, que sempre me acompanham.

A minha família, Rosana, Marcos e Érica. 


\section{AGRADECIMENTOS}

Ao Prof. João Batista de Paiva, pela compreensão, paciência e orientação durante todo o desenvolvimento deste trabalho.

Ao CNPq pelo apoio financeiro.

A todos os colegas do Departamento de Engenharia de Estruturas da EESC/USP, pelo gratificante convívio e amizade, em especial à Júlio Silva, André Christoforo, Daniel Maciel e Alexandre Butler.

Acima de tudo, a DEUS, companheiro fiel, presente em todos os momentos de minha vida. 


\section{Indice}

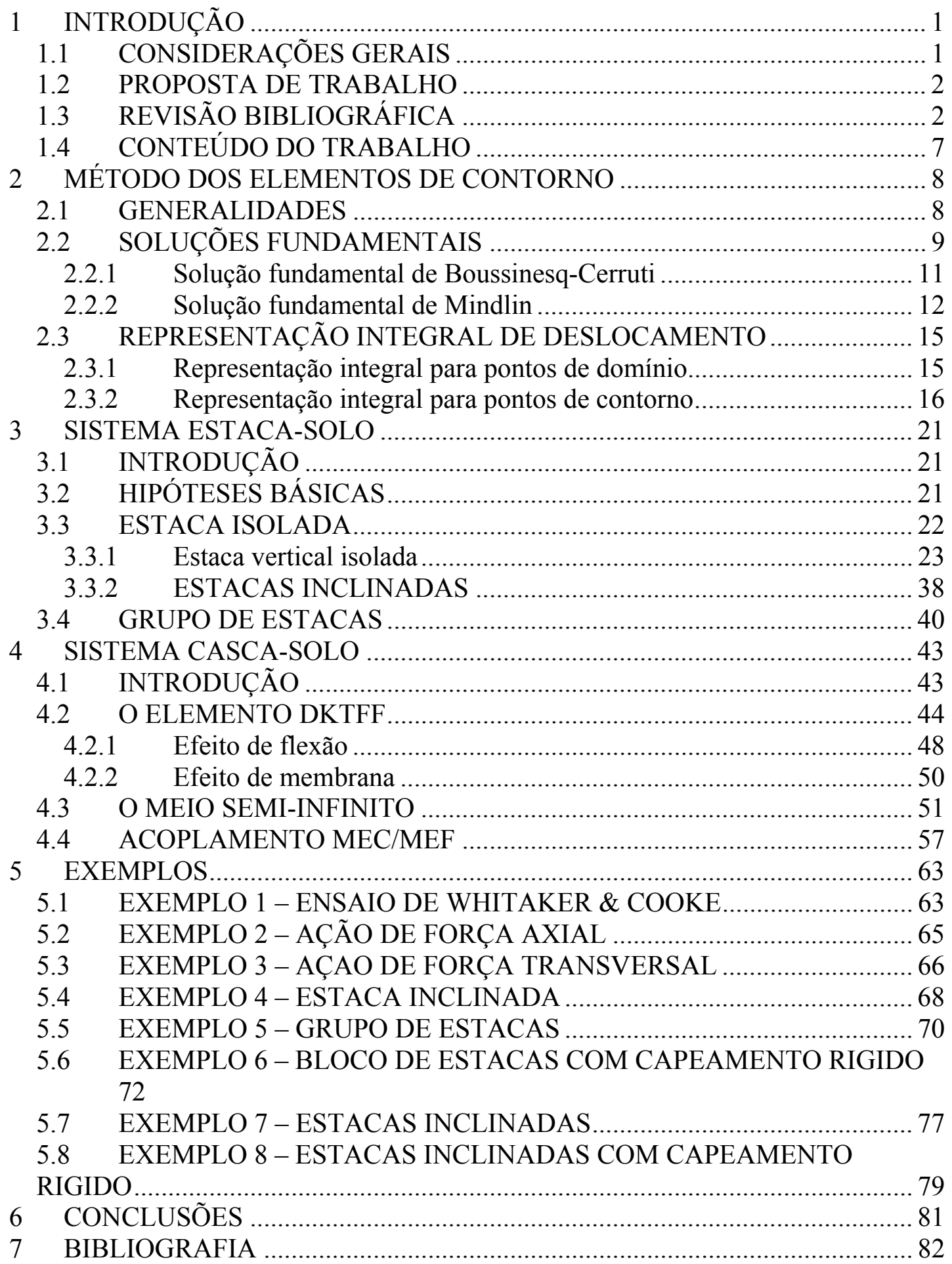




\section{LISTA DE TABELAS}

$\begin{array}{ll}\text { Tabela 5.1 - Deslocamentos na cabeça da estaca } & 65\end{array}$

Tabela 5.2 - Deslocamentos axiais para uma estaca com inclinação $\phi \quad 66$

$\begin{array}{ll}\text { Tabela } 5.3 \text { - Deslocamentos na cabeça da estaca } & 67\end{array}$

Tabela 5.4 - Deslocamentos na cabeça da estaca $\quad 69$

$\begin{array}{ll}\text { Tabela } 5.5 \text { - Deslocamentos horizontais da estaca } & 71\end{array}$

Tabela 5.6 - Coeficientes de mola para as estacas $\quad 75$

Tabela 5.7 - Deslocamentos na estaca (caso a - estacas verticais) 78

Tabela 5.8 - Deslocamentos na estaca (caso b - estacas inclinadas) 78

Tabela 5.9 - Coeficientes de mola para as estacas $\quad 80$ 


\section{LISTA DE FIGURAS}

Figura 2.1 - Problema fundamental de Kelvin $\quad 10$

$\begin{array}{ll}\text { Figura 2.2 - Problema fundamental de Boussinesq-Cerruti } & 11\end{array}$

$\begin{array}{ll}\text { Figura 2.3 - Problema fundamental de Mindlin } & 13\end{array}$

Figura 2.4 - Acréscimo do domínio $\Omega \quad 17$

Figura 3.1 - (a) Discretização do elemento, (b) Forças no topo do elemento;

(c) paramentros nodais de deslocamento; (d) Forças de interação nas direções $\mathrm{X}_{1}$ e $\mathrm{X}_{2}$; (e) Forças de interação na direção $\mathrm{X}_{3}$

Figura 4.1 - Parâmetros nodais e nós auxiliares do elemento DKT 49

Figura 4.2 - Geometria e graus de liberdade do elemento de membrana $\quad 50$

Figura 4.3 - Distribuição das forças de superfície no elemento de contorno $\quad 52$

Figura 4.4 - Integração sobre a célula carregada 53

Figura 4.5 - Forças de superfície e carga nodal equivalente 58

Figura 4.6 - Rede empregada para o solo e a lamina 60

Figura 4.7 - Forças de superfície na interface casca-solo 60

Figura 5.1 - Ensaio de Whitaker \& Cooke 64

Figura 5.2 -Estaca submetida à ação de uma força axial 65

Figura 5.3 -Estaca submetida à ação de uma força transversal 67

Figura 5.2 -Estaca inclinada sujeita a ação de várias forças 68

Figura 5.5 -Gráfico deslocamento x Inclinação 69

Figura 5.6 -Gráfico rotação x Inclinação $\quad 70$

$\begin{array}{ll}\text { Figura 5.7 -Grupo de estacas } & 71\end{array}$

Figura 5.8 -Deslocamento dos grupo de estacas 72

Figura 5.9 -Grupo de estacas com bloco de capeamento rígido 73

$\begin{array}{ll}\text { Figura 5.10 -Grupo de estacas inclinadas com simetria } & 77\end{array}$

Figura 5.11 -Grupo de estacas inclinadas com bloco de capeamento rígido $\quad 79$ 


\section{LISTA DE SIMBOLOS}

$\mathrm{A}_{\mathrm{p}}$ : área da seção transversal da estaca.

$\mathrm{b}_{\mathrm{i}}$ : componentes das forças volumétricas.

c: cota do ponto fonte.

$\mathrm{C}_{\mathrm{ij}}(\mathrm{s})$ : coeficiente de ponderação utilizado na formulação do MEC.

E: módulo longitudinal de elasticidade.

$\mathrm{E}_{\mathrm{p}}$ : módulo longitudinal de elasticidade da estaca.

$\mathrm{E}_{\mathrm{s}}$ : módulo longitudinal de elasticidade do solo.

$F_{1}$ : força lateral externa aplicada na direção $X_{1}$.

$\mathrm{F}_{2}$ : força lateral externa aplicada na direção $\mathrm{X}_{2}$.

G: módulo transversal de elasticidade.

$\mathrm{G}_{\mathrm{s}}$ : módulo transversa de elasticidade do solo.

$\mathrm{g}_{\mathrm{ij}}$ : coeficientes oriundos da integração da solução fundamental.

$I_{p}:$ momento de inércia da seção transversal da estaca.

H: força horizontal que age no topo da estaca.

$\mathrm{Kd}$ : constante elástica utilizada nas soluções fundamentais de Mindlin.

$\mathrm{K}_{\mathrm{R}}$ : coeficiente de flexibilidade do sistema estaca-solo.

L: comprimento da estaca.

M: momento fletor que age no topo da estaca.

$\mathrm{M}_{1}$ : momento externo aplicado em torno do eixo $\mathrm{X}_{2}$.

$\mathrm{M}_{2}$ : momento externo aplicado em torno do eixo $\mathrm{X}_{1}$.

$\mathrm{n}$ : número de elementos, menos um, que constituem uma estaca.

Ne: número de estacas do sistema.

$\mathrm{Ng}$ : número de pontos de Gauss.

$\mathrm{p}_{\mathrm{i}}$ : componentes das forças da interface.

$\mathrm{p}_{\mathrm{ij}}^{*}$ : tensor de forças de superfícies fundamentais.

$\mathrm{Px}_{\mathrm{i}}$ : equação polinomial das forças da interface.

$\mathrm{q}_{\mathrm{ij}}$ : coeficiente da matriz de transformação [Q]. 
$\mathrm{q}_{\mathrm{i}}^{\mathrm{e}}$ : carga distribuída ao longo das estacas.

$r_{f}$ : raio da estaca.

$\mathrm{r}_{\mathrm{i}}$ : distância entre o ponto fonte e o ponto campo.

$\mathrm{R}_{\mathrm{i}}$ : distância entre o ponto fonte fictício e o ponto campo.

$\mathrm{U}$ : funcional de energia potencial de deformação do elemento.

$\mathrm{u}_{\mathrm{ap}}$ : equação polinomial dos deslocamentos nodais na direção $\mathrm{X}_{1}$.

$\mathrm{u}_{\mathrm{i}}$ : representa as componentes de deslocamentos.

$\mathrm{u}_{\mathrm{ij}}^{*}$ : tensor de deslocamentos fundamentais.

V: força vertical externa aplicada no topo da estaca.

$\mathrm{V}_{\mathrm{ap}}$ : equação polinomial dos deslocamentos nodais na direção $\mathrm{X}_{2}$.

$\mathrm{W}_{\mathrm{ap}}$ : equação polinomial dos deslocamentos nodais na direção $\mathrm{X}_{3}$.

$\mathrm{W}_{\mathrm{m}}$ : peso de ponderação da integração de Gauss.

$\mathrm{x}_{\mathrm{i}}(\mathrm{p})$ : coordenadas do ponto campo.

$\mathrm{x}_{\mathrm{i}}(\mathrm{s})$ : coordenadas do ponto fonte.

$\mathrm{X}_{\mathrm{i}}\left(\mathrm{s}^{\prime}\right)$ : coordenadas do ponto fonte fictício.

Z: cota do ponto campo.

$\delta(\mathrm{s}, \mathrm{p})$ : distribuição Delta de Dirac.

$\delta_{\mathrm{ij}}$ : delta de Kronecker.

$\varepsilon_{\mathrm{ij}}^{*}:$ tensor de deformações fundamentais.

$\varepsilon_{\mathrm{ijj}}$ : representa as componentes de deformação no sistema global.

$\lambda$ : ângulo que a projeção da estaca em planta faz com o eixo $\mathrm{X}_{1}$.

v: coeficiente de Poisson

$v_{\mathrm{s}}$ : coeficiente de Poisson do solo.

$\sigma_{\mathrm{b}}$ : tensão normal que age na base do elemento.

$\sigma_{\mathrm{ij}}$ : representa as componentes de tensão no sistema global.

$\sigma_{\mathrm{ij}}^{*}$ : tensor de tensões fundamentais.

$\xi$ : cota adimensional do elemento.

$\tau_{\mathrm{pi}}$ : tensões cisalhantes que agem no fuste do elemento.

$\Gamma_{\mathrm{e}}$ : contorno onde são aplicadas as forças de interação. 
$\bar{\Gamma}$ : contorno superficial fictício.

$\Gamma_{\varepsilon}:$ contorno volumétrico fictício.

$\Gamma^{*}$ : contorno de um meio infinito.

$\Gamma$ : contorno finito de um corpo genérico.

$\Gamma_{1}$ : região do contorno de um corpo que contém as forças de superfícies prescritas.

$\Gamma_{2}$ : região do contorno de um corpo que contém os deslocamentos prescritos.

$\Omega_{\varepsilon}:$ domínio volumétrico fictício.

$\Omega^{*}$ : domínio infinito associado ao problema fundamental.

$\Omega$ : domínio finito dos corpos.

$\Omega$ ': funcional de energia potencial do carregamento externo.

П: funcional de energia potencial total do elemento.

$\{F\}$ : vetor de cargas externas.

[G]: matriz de coeficientes de influência do maciço de solos.

[I] : matriz identidade.

$\left[\mathrm{K}_{\mathrm{c}}\right]$ : matriz de rigidez do elemento.

$\{\mathrm{M}\}$ : vetor auxiliar de rotação.

$\{\mathrm{P}\}$ : vetor de forças da interface.

$\left\{\mathrm{P}_{\mathrm{p}}\right\}$ : vetor de forças da interface que agem sobre a estaca.

$\left\{\mathrm{P}_{\mathrm{s}}\right\}$ : vetor de forças da interface que agem sobre o solo.

[Q]: matriz de transformação das cargas distribuídas na interface em cargas nodais.

$\left[\mathrm{Q}_{\mathrm{ij}}\right]$ : sub-matriz de coeficientes da matriz $[\mathrm{Q}]$ referentes a grupos de estacas.

[R]: matriz de rotação.

[U*]: matriz das soluções fundamentais.

$\{\mathrm{u}\}$ : vetor de deslocamentos dos pontos nodais.

$\left\{u_{p}\right\}$ : vetor de deslocamentos nodais da estaca.

$\left\{u_{s}\right\}$ : vetor de deslocamentos nodais do solo.

$\left\{\mathrm{X}^{\mathrm{p}}\right\}$ : vetor de coordenadas globais dos pontos campo.

$\left\{\mathrm{x}^{\mathrm{s}}\right\}$ : vetor de coordenadas globais dos pontos fonte.

$\left\{\overline{\mathrm{x}}^{\mathrm{p}}\right\}$ : vetor de coordenadas locais dos pontos campo. 
LISTA DE ABREVIATURAS E SIGLAS

$\begin{array}{ll}\text { DKT } & \text { Discrete Kirchoff Theory. } \\ \text { HSM } & \text { Hybrid Stress Model. } \\ \text { MEC } & \text { Método dos Elementos Finitos. } \\ \text { MEF } & \text { Método dos Elementos de Contorno. } \\ \text { FF } & \text { Formulação Livre }\end{array}$




\section{RESUMO}

OSHIMA, S.T. (2004). Uma combinação MEC/MEC para análise da interação de estacas inclinadas e o solo. São Carlos, 2004. 84p. Dissertação (Mestrado) Escola de Engenharia de São Carlos, Universidade de São Paulo.

O presente trabalho apresenta uma formulação mista do MEC (método dos elementos de contorno) e o MEF (método dos elementos finitos). Nessa formulação, as estacas são modeladas através do MEF como elementos de barra e o solo através do MEC, como um meio contínuo, elástico linear, isótropo e homogêneo, utilizando as soluções fundamentais de MINDLIN (1936). Os sistemas de equações do solo e das estacas para elementos verticais são apresentados como uma combinação de ambos, originando um único sistema final de equações. Apresentam-se também as modificações necessárias para um sistema composto por estacas inclinadas. Após a resolução do sistema final, obtém-se os deslocamentos e as tensões de contato soloestaca. A seguir, apresentam-se alguns exemplos numéricos obtidos a partir da formulação proposta e compara-se com modelos de outros autores.

Palavras-chave: Estacas, interação solo-estrutura, método dos elementos de contorno, método dos elementos finitos. 


\section{ABSTRACT}

OSHIMA, S.T. (2004). A combination BEM/FEM for analysis of the interaction of inclinated piles and the soil. São Carlos, 2004. 84p. Dissertação (Mestrado) Escola de Engenharia de São Carlos, Universidade de São Paulo.

This work presents a hybrid formulation of BEM (Boundary Elements Method) and FEM (Finite Elements Method). In that formulation, the piles are modeled through FEM as bar elements and the soil through BEM, as an isotropic, homogeneous, semi-infinite and linear-elastic continuum, using the fundamental solutions of MINDLIN (1936). The systems of equations of the soil and of the piles for vertical elements are presented as a combination of both, originating a single final system of equations. Some modifications are accomplished for the system of inclinated piles. After the resolution of the final system, the displacements and the contact tensions between soil and pile are obtained. Numeric examples are obtained starting from the proposed formulation and to proceed they are compared with other authors' models.

Keywords: Piles, soil-structure interaction, Boundary Elements Method, Finite Element Method. 


\section{INTRODUÇÃO}

\subsection{CONSIDERAÇÕES GERAIS}

Atualmente a interação solo-estrutura tem sido um assunto muito discutido entre pesquisadores do mundo todo. O comportamento real de um sistema soloestrutura é um assunto complexo, envolvendo muitas variáveis e hipóteses. Devido a essa dificuldade uma solução promissora consiste na simulação numérica.

Vários pesquisadores têm se dedicado à analise do problema da interação solo-estrutura, utilizando formulações mistas de técnicas consagradas como o MEF (método dos elementos finitos) e técnicas relativamente recentes, como o MEC (método dos elementos de contorno), aproveitando as vantagens de ambas as ferramentas.

O MEF é uma ferramenta já consagrada no meio técnico, sendo o método numérico mais utilizado nos problemas de engenharia. Já o MEC é uma técnica muito utilizada na modelagem de meios infinitos ou semi-infinitos, e tem como característica a redução do número de equações e variáveis envolvidas, diminuindo consideravelmente o tempo de processamento do problema. 


\subsection{PROPOSTA DE TRABALHO}

$\mathrm{O}$ presente trabalho tem como objetivo principal o estudo e o desenvolvimento de um código computacional que possibilite a análise da interação solo-estrutura através de uma formulação mista entre o MEC e o MEF.

Nesta formulação, as estacas serão modeladas através do método dos elementos finitos como elementos de barra. Inicialmente, serão consideradas apenas estacas verticais isoladas. Posteriormente, o estudo será direcionado para grupo de estacas e estacas inclinadas.

O solo será considerado um meio semi-infinito, homogêneo, contínuo, isótropo e elástico linear. Sua influência será considerada através do MEC utilizando as equações de Mindlin.

\subsection{REVISÃO BIBLIOGRÁFICA}

Com a evolução dos computadores, os métodos numéricos se transformaram em uma ferramenta cada vez mais utilizada pelos pesquisadores. Atualmente um dos grandes campos de pesquisa em que se utiliza essa ferramenta é a interação soloestrutura.

$\mathrm{Na}$ realidade, o interesse por pesquisas sobre o comportamento do solo é bastante antigo, porém como avanço dos métodos numéricos, se tornaram cada vez mais freqüente.

Dentre os vários modelos propostos sobre o comportamento do solo, pode-se destacar três deles: 
a) Modelo de Winkler

WINKLER (1867), propôs que cargas aplicadas na superfície do solo geram deslocamentos somente no ponto de aplicação da mesma, ou seja, desconsidera o efeito da continuidade do meio.

b) Modelo do meio contínuo

Prevê o deslocamento em pontos distintos ao ponto de aplicação de carga, considerando o efeito da continuidade do meio, inicialmente desprezada por Winkler. Vários autores utilizaram este modelo em suas pesquisas. Flamant analisou linhas de cargas normais à superfície num semi-espaço, descritas em TIMOSHENKO \& GOODIER (1970). SELVADURAI (1979) estudou o comportamento dos deslocamentos na superfície de um semi-espaço finito sob um carregamento normal uniforme de largura finita, através de uma aproximação da integral de Fourier ou através do método as superposição, descrito anteriormente por SNEDDON (1958). WATSON (1944) analisou através do método da superposição, o comportamento de uma região circular sob um carregamento dado por uma pressão constante uniforme. O integrando é ponderado por funções de Bessel. CERRUTI (1882) apresentou soluções para o problema de cargas normais a superfície de um semi-espaço, homogêneo, isótropo e elástico linear. BOUSSINESQ (1885) fez uma análise semelhante, para cargas normais à superfície. MINDLIN (1936), generalizou o problema, analisando o comportamento do semi espaço sujeito a cargas unitárias em qualquer posição do seu domínio. Os modelos de Boussinesq-Cerruti e de Mindlin serão apresentados com maiores detalhes no capitulo 2 .

c) Modelo de dois parâmetros

É um modelo definido através de duas constantes elásticas independentes, visando reduzir a descontinuidade do modelo de proposto por Winkler.

Baseado no modelo de dois parâmetros, Selvadurai cita alguns trabalhos de outros pesquisadores: 
c1) Modelo de Filonenko-Borodich: Através da utilização de uma membrana delgada tensionada entre as molas, a continuidade é garantida, incorporando na equação diferencial, a contribuição da tensão da membrana e o coeficiente de base elástica de Winkler.

c2) Modelo de Pasternack: De maneira análoga ao principio anterior, mas com a continuidade entre as molas simulada através da utilização de uma camada flexível apenas ao cisalhamento, fazendo com que a equação diferencial seja afetada pelo módulo de elasticidade transversal da camada e pelo coeficiente da base elástica.

c3) Modelo de Hetényi: Neste modelo, a continuidade do meio é simulada através de uma placa elástica na interação entre as molas, visto que a equação integral tem uma parcela da equação de placas e outra referente a base elástica de Winkler.

c4) Modelo de Vlasov: É um modelo híbrido, oriundo das restrições sobre as possíveis distribuições de deslocamentos. Através do método variacional, obtêm-se as equações diferenciais para o maciço de solo. Através deste modulo, obtêm-se resultados semelhantes pelo método dos dois parâmetros.

Com relação à interação estaca-solo, vários trabalhos foram apresentados, utilizando o modelo de Winkler. MATLOCK \& REESE (1961), propuseram um método de cálculo de uma estaca solicitada por uma força horizontal e um momento na superfície do terreno. Neste trabalho, a variação do módulo de reação horizontal do maciço de solo, em função da profundidade, foi analisado de duas formas, uma polinomial e outra exponencial. NAVDOCKS (1962), através do trabalho proposto por MATLOCK \& REESE (1956), onde o módulo de reação horizontal do maciço de 
solo foi admitido linearmente crescente com a profundidade, apresentou soluções para o problema de uma estaca submetida a ação de uma carga horizontal e um momento fletor. BROMS (1965), considerando módulos de reação horizontal do maciço tanto constante, como linearmente crescente com a profundidade, trabalhando no estado limite de ruptura, obteve soluções analíticas para estacas rígidas e flexíveis, sujeitas a carregamentos laterais no topo. DAVINSON \& ROBINSON (1965), propuseram um estudo do problema da flexão e flambagem em estacas parcialmente enterradas, sujeito aos efeitos de momento, carga lateral e axial, considerados separadamente. Neste trabalho, a reação do maciço de solos é considerada constante ou linearmente crescente com a profundidade.

Um dos estudos mais antigos sobre o comportamento de estacas deve-se a POULOS \& DAVIS (1968). Nesta pesquisa, estudaram o problema de uma estaca cilíndrica incompressível isolada, submetida a ação de uma carga axial, imersa em um meio semi infinito isótropo e homogêneo. A tensão de cisalhamento ao longo da estaca foi admitida constante e na base alongada, apenas a tensão axial. Neste problema foi empregada a solução fundamental de Mindlin para deslocamentos verticais. As integrais foram resolvidas analiticamente ao longo da estaca e numericamente no sentido circunferencial.

Uma extensão desta formulação foi proposta por POULOS (1968). Neste trabalho foram realizadas análises de grupos de estacas. Inicialmente, foi feito um estudo da interação de duas estacas idênticas, com carregamentos semelhantes e a partir dos resultados obtidos, o método foi aplicado a um grupo de estacas, considerando a superposição elástica da influência de todos os elementos do grupo, tomados sempre dois a dois.

Uma nova formulação, agora considerando a compressibilidade da estaca foi proposta por MATTES \& POULOS (1969). Nela a equação diferencial dos deslocamentos verticais são expressos na forma de diferenças finitas.

POULOS (1971a,b) apresenta uma formulação similar à anterior para uma estaca vertical submetida a uma força horizontal e momento, utilizando as equações de Mindlin para modelar o maciço de solo. A estaca foi discretizada pelo método das 
diferenças finitas. Logo a seguir, estendeu esta formulação para o caso de grupo de estacas.

POULOS \& MADHAV (1971), estudaram o problema de estacas isoladas inclinadas submetidas primeiramente à um carregamento axial e posteriormente á um carregamento normal e momento fletor, concluindo que os deslocamentos são praticamente independentes do ângulo de inclinação da estaca.

No Departamento de Engenharia de Estruturas - SET/EESC, foram publicados vários trabalhos cujo tema principal é interação solo-estrutura. Dentre eles pode-se citar o trabalho de FERRO (1993) que apresentou uma formulação mista do MEC/MEF para a análise da interação solo-estrutura. O solo foi considerado um meio semi-infinito, homogêneo, continuo, isótropo e elástico linear, utilizando as equações de Mindlin. A estaca foi modelada por um elemento de barra. PAIVA (1993), estudou o problema da interação placa-estaca-solo através do método dos elementos de contorno.

MENDONÇA (1997) estudou a interação placa-estaca-solo. O solo foi modelado pelo MEC utilizando as equações de Mindlin. A placa foi discretizada utilizando elementos triangulares (DKT e HSM) e a estaca por elementos de barra. Neste estudo, admitiu-se que as forças interação variam linearmente nas células e as forças de cisalhamento na estaca são aproximadas por um polinômio quadrático. Admite-se que na base da estaca atue somente uma tensão axial constante.

MATOS FILHO (1999), desenvolveu um modelo de estacas isoladas ou grupo de estacas submetida à ação de um carregamento horizontal e momento em duas direções e carregamento vertical. O solo foi modelado pelo MEC, utilizando as equações fundamentais de Mindlin. As estacas foram modeladas como elementos de barra. Os deslocamentos transversais foram aproximados por um polinômio de $4^{\mathrm{a}}$ ordem, e os deslocamentos verticais, por um polinômio cúbico. As forças de interação nas direções $\mathrm{X}_{1}$ e $\mathrm{X}_{2}$ são aproximadas por um polinômio cúbico e na direção $X_{3}$, por um polinômio quadrático. A tensão na base da estaca é admitida constante. O modelo proposto por Matos Filho será abordado com mais detalhes no capitulo3. ALMEIDA (2003) estudou o problema da interação solo-estrutura através 
de uma combinação mista entre o MEC e o MEF. A estrutura foi discretizada por elementos reticulares e laminares, o solo foi considerado um meio não homogêneo estratificado com camada de cota indeslocável.

\subsection{CONTEÚDO DO TRABALHO}

No capitulo 2 apresenta-se a teoria do MEC, envolvendo as equações de elasticidade linear para corpos tridimensionais, as equações integrais de contorno e as soluções fundamentais de Kelvin, Mindlin, e Boussinesq-Cerruti.

No capitulo 3, é desenvolvida toda a formulação e acoplamento entre MEC e o MEF para o análise de estacas imersas num meio semi-infinito, homogêneo, continuo, isótropo e elástico linear, utilizando as equações de Mindlin.

No capitulo 4, apresenta-se o sistema casca-solo. No capítulo 5 são apresentados exemplos numéricos para a avaliação de estacas isoladas ou em grupo, com ou sem capeamento rígido, inclinadas ou não.

Já o capitulo 6 traz as conclusões finais decorrentes do emprego do método dos elementos de contorno e o método dos elementos finitos na interação soloestrutura. 


\section{MÉTODO DOS ELEMENTOS DE CONTORNO}

Neste capítulo são abordados os problemas elásticos fundamentais em sólidos tridimensionais, bem como as equações integrais para a utilização do MEC, relacionando os problemas elásticos reais e fundamentais.

\subsection{GENERALIDADES}

Através do método dos elementos de contorno, utilizando-se as relações básicas da teoria da elasticidade, é possível obter soluções da análise de problemas elásticos.

O MEC é derivado de uma representação integral que envolve dois tipos de problemas: o primeiro, associado ao problema fundamental, considerando-se um domínio infinito $\Omega *$ e contorno $\Gamma^{*}$, envolvendo as variáveis $\mathrm{u}^{*}{ }_{\mathrm{ij}}$ (tensor de deslocamentos fundamentais de $2^{\mathrm{a}}$ ordem), $\mathrm{p}^{*}{ }_{\mathrm{ij}}$ (tensor de forças de superfície fundamentais de ordem 2), $\sigma^{*}{ }_{i j k}$ (tensor de tensões fundamentais de ordem 3) e $\varepsilon^{*}{ }_{i j k}$ (tensor de deformações fundamentais de $3^{\mathrm{a}}$ ordem); o segundo, associado ao problema real, definido em um domínio finito $\Omega$, contido em $\Omega^{*}$, envolvendo as componentes $\mathrm{u}_{\mathrm{i}}, \mathrm{p}_{\mathrm{i}}, \sigma_{\mathrm{ij}}$ e $\varepsilon_{\mathrm{ij}}$, caracterizado pelas condições de contorno naturais e essenciais. 
Entende-se por solução fundamental, a resposta em um ponto "p" (ponto campo), observado na direção $\mathrm{j}$, devido a aplicação de uma carga unitária concentrada no ponto "s" (ponto fonte) na direção i.

A equação governante dos sólidos elásticos, também conhecida como equação de Navier, para uma carga unitária, pode ser escrita como:

$$
\frac{1}{1-2 v} u_{i k, j k}^{*}+u_{i j, k k}^{*}+\frac{1}{G} \delta(s, p) \delta_{i j}=0
$$

onde:

v: constante elástica conhecida como coeficiente de Poisson

G: módulo de elasticidade transversal

$\delta_{\mathrm{ij}}$ : delta de Kronecker $(0$ se $\mathrm{i} \neq \mathrm{j} ; 1$ se $\mathrm{i}=\mathrm{j})$

$\delta(s, p)$ distribuição Delta de Dirac

A solução fundamental em deslocamentos $\mathrm{u}^{{ }_{\mathrm{ij}}}$ depende das características do domínio e do contorno da região onde o problema elástico está inserido. Assim, a seguir, apresentam-se as soluções fundamentais de Kelvin, Boussinesq-Cerruti e Mindlin.

\subsection{SOLUÇÕES FUNDAMENTAIS}

A solução fundamental de Kelvin para problemas elásticos definidos em um domínio tridimensional infinito $\Omega^{*}$, homogêneo, isótropo e elástico-linear, submetido a ação de cargas unitárias concentradas, foi proposta por LOVE (1944). 


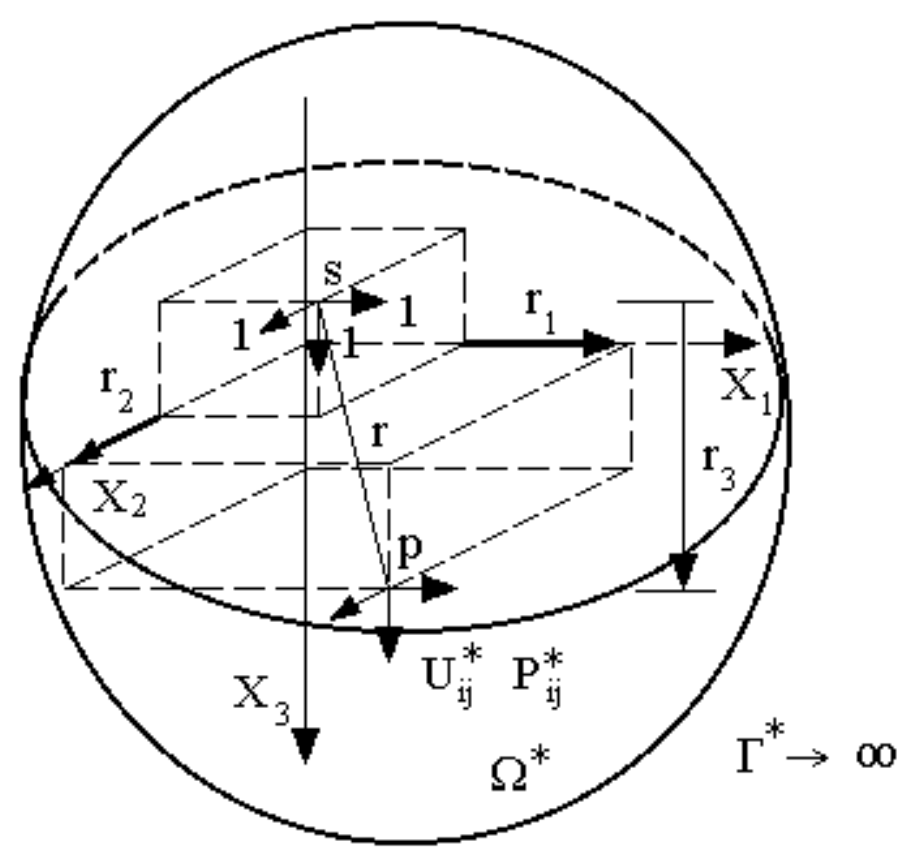

Figura 2.1 - Problema fundamental de Kelvin

As soluções fundamentais de deslocamento e forças de superfície em termos de seus respectivos tensores são dadas abaixo:

$$
\begin{aligned}
& u_{i j}^{*}=\frac{1+v}{8 \pi E r(1-v)}\left[(3-4 v) \delta_{i j}+r,{ }_{j} r,_{i}\right] \\
& p_{i j}^{*}=\frac{-1}{8 \pi E r^{2}(1-v)}\left\{r,{ }_{i} n_{i}\left[(1-2 v) \delta_{i j}+3 r_{{ }_{i}} r_{j}\right]-(1-2 v)\left(n_{j} r,_{i}-n_{i} r,{ }_{j}\right)\right\}
\end{aligned}
$$

onde:

$$
\begin{aligned}
& r=\left(r_{i} r_{i}\right)^{1 / 2} \\
& r_{i}=x_{i}(p)-x_{i}(s) \\
& r_{, i}=\partial r_{i} / \partial r=r_{i} / r
\end{aligned}
$$




\subsubsection{Solução fundamental de Boussinesq-Cerruti}

CERRUTI (1882) analisou o problema de cargas concentradas tangenciais aplicadas no plano de contorno $\Gamma$ (limitado pelo plano $\mathrm{X}_{3}=0$ ) de sólidos tridimensionais considerando o domínio $\Omega^{*}$ semi-infinito, homogêneo, isótropo, elástico-linear e por hipótese, livre de forças de superfície no contorno. BOUSSINESQ (1885) fez uma análise semelhante levando em consideração apenas cargas concentradas normais a superfície de contorno.

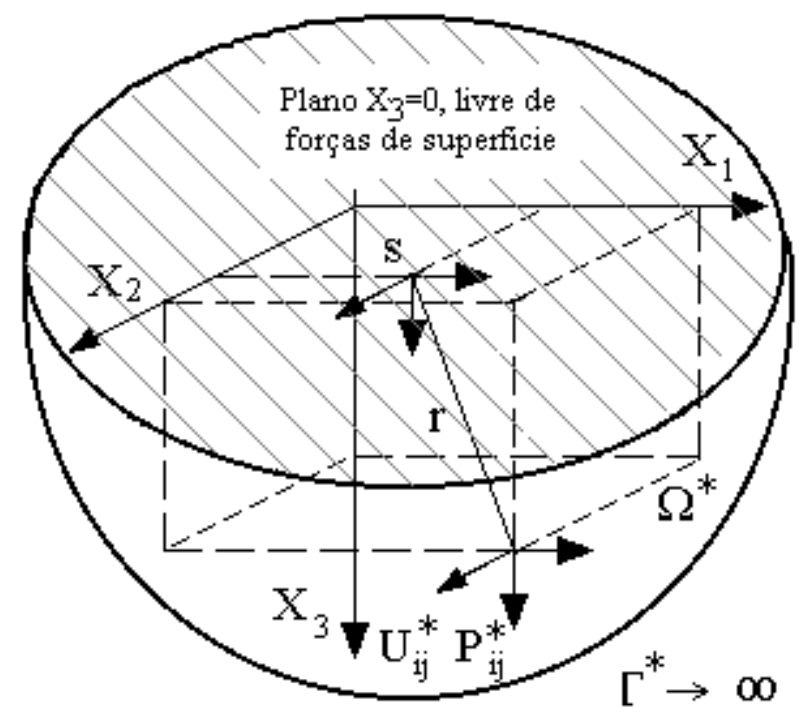

Figura 2.2 - Problema fundamental de Boussinesq-Cerruti

Particularmente, para $\mathrm{x}_{3}(\mathrm{p})=0$, as soluções fundamentais de deslocamento são apresentadas abaixo:

$$
\begin{aligned}
& u_{11}^{*}=\frac{(1-v)+v r^{2},}{2 \pi G r} \\
& u_{12}^{*}=u_{21}^{*}=\frac{v r,_{1} r,_{2}}{2 \pi G r}
\end{aligned}
$$




$$
\begin{aligned}
& u_{13}^{*}=\frac{(0,5-v)+v r_{,_{1}}}{2 \pi G r} \\
& u_{22}^{*}=\frac{(1-v)+v r^{2},_{2}}{2 \pi G r} \\
& u_{23}^{*}=\frac{(0,5-v)+v r_{,_{2}}}{2 \pi G r} \\
& u_{31}^{*}=\frac{(v-0,5)+v r_{1}}{2 \pi G r} \\
& u_{32}^{*}=\frac{(v-0,5)+v r_{, 2}}{2 \pi G r} \\
& u_{33}^{*}=\frac{1-v}{2 \pi G r}
\end{aligned}
$$

\subsubsection{Solução fundamental de Mindlin}

Mindlin apresentou seu trabalho, soluções fundamentais para problemas elásticos definidos em um domínio tridimensional semi-infinito, homogêneo, isótropo e elástico-linear, assumindo que o plano definido em $\mathrm{x}_{3}=0$, esteja livre de forças de superfície e considerado como superfície de contorno.

Diferentemente do trabalho de Boussinesq-Cerruti, as equações de Mindlin permitem a colocação de pontos fontes em planos diferentes daquele definido por $\mathrm{X}_{3}=0$. 


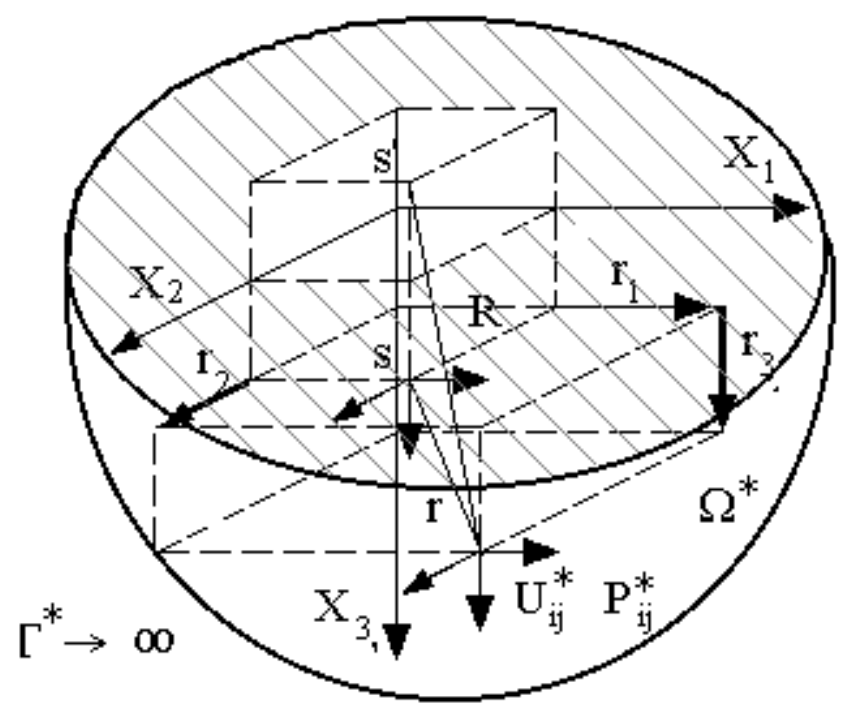

Figura 2.3 - Problema fundamental de Mindlin

As equações para deslocamentos fundamentais são descritas a seguir:

$$
\begin{aligned}
& \mathrm{u}_{11}=\mathrm{Kd}\left\{\frac{3-4 v}{\mathrm{r}}+\frac{1}{\mathrm{R}}+\frac{\mathrm{r}_{1}^{2}}{\mathrm{r}^{3}}+\frac{(3-4 v) \mathrm{r}_{1}^{2}}{\mathrm{R}^{3}}+\frac{2 \mathrm{cz}}{\mathrm{R}^{3}}\left(1-\frac{3 \mathrm{r}_{1}^{2}}{\mathrm{R}^{2}}\right)+\frac{4(1-v)(1-2 v)}{\mathrm{R}+\mathrm{R}_{3}}\left(1-\frac{\mathrm{r}_{1}^{2}}{\mathrm{R}\left(\mathrm{R}+\mathrm{R}_{3}\right)}\right)\right\} \\
& \mathrm{u}_{12}=\mathrm{Kdr}_{1} \mathrm{r}_{2}\left\{\frac{1}{\mathrm{r}^{3}}+\frac{3-4 v}{\mathrm{R}^{3}}-\frac{6 \mathrm{cz}}{\mathrm{R}^{5}}-\frac{4(1-v)(1-2 v)}{\left(\mathrm{R}+\mathrm{R}_{3}\right)^{2}}\right\} \\
& \mathrm{u}_{13}=\mathrm{Kdr}_{1}\left\{\frac{\mathrm{r}_{3}}{\mathrm{r}^{3}}+\frac{(3-4 v) \mathrm{r}_{3}}{\mathrm{R}^{3}}-\frac{6 \mathrm{czR} \mathrm{R}_{3}}{\mathrm{R}^{5}}+\frac{4(1-v)(1-2 v)}{\mathrm{R}+\mathrm{R}_{3}}\right\} \\
& \mathrm{u}_{21}=\mathrm{Kdr}_{1} \mathrm{r}_{2}\left\{\frac{1}{\mathrm{r}^{3}}+\frac{3-4 v}{\mathrm{R}^{3}}-\frac{6 c z}{\mathrm{R}^{5}}-\frac{4(1-v)(1-2 v)}{\left(\mathrm{R}+\mathrm{R}_{3}\right)^{2}}\right\} \\
& \mathrm{u}_{22}=\mathrm{Kd}_{2}\left\{\frac{3-4 v}{\mathrm{r}}+\frac{1}{\mathrm{R}}+\frac{\mathrm{r}_{2}^{2}}{\mathrm{r}^{3}}+\frac{(3-4 v) \mathrm{r}_{2}^{2}}{\mathrm{R}^{3}}+\frac{2 \mathrm{cz}}{\mathrm{R}^{3}}\left(1-\frac{3 \mathrm{r}_{2}^{2}}{\mathrm{R}^{2}}\right)+\frac{4(1-v)(1-2 v)}{\mathrm{R}+\mathrm{R}_{3}}\left(1-\frac{\mathrm{r}_{2}^{2}}{\mathrm{R}\left(\mathrm{R}+\mathrm{R}_{3}\right)}\right)\right\} \\
& \mathrm{u}_{23}=\operatorname{Kdr}\left\{\frac{\mathrm{r}_{3}}{\mathrm{r}^{3}}+\frac{(3-4 v) \mathrm{r}_{3}}{\mathrm{R}^{3}}-\frac{6 c z R_{3}}{\mathrm{R}^{5}}+\frac{4(1-v)(1-2 v)}{\mathrm{R}+\mathrm{R}_{3}}\right\}
\end{aligned}
$$




$$
\begin{aligned}
& \mathrm{u}_{31}=\mathrm{Kdr}_{1}\left\{\frac{\mathrm{r}_{3}}{\mathrm{r}^{3}}+\frac{(3-4 v) \mathrm{r}_{3}}{\mathrm{R}^{3}}+\frac{6 c z R_{3}}{\mathrm{R}^{5}}-\frac{4(1-v)(1-2 v)}{\mathrm{R}+\mathrm{R}_{3}}\right\} \\
& \mathrm{u}_{32}=\mathrm{Kdr}_{2}\left\{\frac{\mathrm{r}_{3}}{\mathrm{r}^{3}}+\frac{(3-4 v) r_{3}}{\mathrm{R}^{3}}+\frac{6 c z R_{3}}{\mathrm{R}^{5}}-\frac{4(1-v)(1-2 v)}{\mathrm{R}+\mathrm{R}_{3}}\right\} \\
& \mathrm{u}_{33}=\operatorname{Kd}\left\{\frac{\mathrm{r}_{3}^{2}}{\mathrm{r}^{3}}+\frac{(3-4 v)}{\mathrm{r}}+\frac{6 \mathrm{czR} \mathrm{R}_{3}^{2}}{\mathrm{R}^{5}}+\frac{8(1-v)^{2}(3-4 v)}{\mathrm{R}}+\frac{(3-4 v) \mathrm{R}_{3}^{2}-2 \mathrm{cz}}{\mathrm{R}^{3}}\right\}
\end{aligned}
$$

onde:

$$
\begin{aligned}
& \mathrm{R}=\left(\mathrm{R}_{\mathrm{i}} \mathrm{R}_{\mathrm{i}}\right)^{1 / 2} \\
& \mathrm{R}_{\mathrm{i}}=\mathrm{x}_{\mathrm{i}}(\mathrm{p})-\mathrm{x}_{\mathrm{i}}{ }^{\prime}(\mathrm{s}) \\
& \mathrm{c}=\mathrm{x}_{3}(\mathrm{~s})>0 \\
& \mathrm{z}=\mathrm{x}_{3}(\mathrm{p})>0 \\
& K d=\frac{1+\mathrm{v}}{8 \pi E(1-\mathrm{v})}
\end{aligned}
$$

Nota-se que as soluções fundamentais de Mindlin podem ser escritas como uma somatória da solução fundamental de Kelvin acrescida de uma parcela referente a influência do plano de contorno $\mathrm{X}_{3}=0$. Assim, afastando os pontos "s" e " $p$ " do plano de contorno, isto é, fazendo $\mathrm{c} \rightarrow \infty$ e $\mathrm{z} \rightarrow \infty$, tem-se $\mathrm{R}_{3} \rightarrow \infty$ e $\mathrm{R} \rightarrow \infty$, e como conseqüência, a parcela referente a influência do contorno tende a zero, chegando-se finalmente à solução de Kelvin.

Agindo agora de maneira contrária, isto é, aproximando os postos "s" e "p" do plano definido por $X_{3}=0$, nota-se que as soluções fundamentais tendem às soluções de Boussinesq-Cerruti, apresentadas no item anterior. Desta forma, conclui- 
se que as equações de Boussinesq-Cerruti são casos particulares da solução de Mindlin, para cargas aplicadas na superfície do sólido.

\subsection{REPRESENTAÇÃO INTEGRAL DE DESLOCAMENTO}

A representação integral para o campo de deslocamentos pode ser dividida em duas partes: a primeira chamada de equação integral para pontos de domínio, é caracterizada pelo ponto fonte no domínio; e a segunda, conhecida como equação integral para pontos de contorno, quando o ponto fonte se encontra no contorno.

\subsubsection{Representação integral para pontos de domínio}

A representação integral para pontos de domínio pode ser obtida através de vários métodos, como a técnica dos resíduos ponderados, o teorema da divergência ou o teorema da reciprocidade (teorema de Betti). Por ser bastante comum entre as técnicas numéricas, o método dos resíduos ponderados será empregado neste trabalho.

A equação de equilíbrio de um corpo é dada por:

$$
\sigma_{i j, j}+b_{i}=0 \quad(i, j=1,2,3)
$$

Para garantir o equilíbrio do corpo, faz-se necessário obter a solução das equações diferencias de equilíbrio. Multiplicando a equação (2.29) por uma função ponderadora $\mathrm{u}_{\mathrm{ij}}{ }_{\mathrm{j}} \mathrm{e}$ integrando-se no domínio $\Omega^{*}$, temos:

$$
\int_{\Omega}\left(\sigma_{i j},{ }_{j}+b_{i}\right) u_{i j}^{*} d \Omega=0 \quad(i, j=1,2,3)
$$

onde: 
$\mathrm{u}_{\mathrm{ij}}$ : $\quad$ solução fundamental relativo a uma carga unitária na direção “i” e deslocamento na direção "j"

$b_{i}$ : força volumétrica na direção "i”"

Integrando-se duas vezes por partes a equação (2.30), temos:

$$
\int_{\Omega} \sigma_{i j}^{*}, u_{j} d \Omega+\int_{\Omega} u_{i j}^{*} b_{i} d \Omega=\int_{\Gamma} p_{i j}^{*} u_{i} d \Gamma-\int_{\Gamma} u_{i j}^{*} p_{i} d \Gamma
$$

Lembrando que a integração de uma função delta de Dirac ao longo de um domínio fornece um valor unitário:

$$
\int_{\Omega} \delta(s, p) \delta_{i j} u_{j} d \Omega=\delta_{i j} u_{j}=u_{i}
$$

Substituindo (2.32) em (2.31), temos:

$$
u_{i}=-\int_{\Gamma} p_{i j}^{*} u_{j} d \Gamma+\int_{\Gamma} u_{i j}^{*} p_{j} d \Gamma+\int_{\Omega} u_{i j}^{*} b_{j} d \Omega
$$

Esta equação é chamada de Identidade Somigliana e é válida somente para os pontos de domínio $\Omega$.. Ela fornece as componentes de deslocamento no ponto fonte, situado no interior do domínio $\Omega$, uma vez conhecida as componentes $\mathrm{u}_{\mathrm{j}}$ e $\mathrm{p}_{\mathrm{j}}$ situadas no contorno.

\subsubsection{Representação integral para pontos de contorno}

Retomando a equação (2.31) e dividindo-se o contorno em $\Gamma_{1}$ e $\Gamma_{2}$, onde se aplicam as condições de contorno essenciais (deslocamentos prescritos, $u=\bar{u}$ ) e naturais (forças prescritas, $\mathrm{p}=\overline{\mathrm{p}}$ ), obtêm-se:

$$
\int_{\Omega} \sigma_{i j}^{*},{ }_{j} u_{i} d \Omega+\int_{\Omega} u_{i j}^{*} b_{i} d \Omega=-\int_{\Gamma_{1}} u_{i j}^{*} p_{i} d \Gamma-\int_{\Gamma_{2}} u_{i j}^{*} \bar{p}_{i} d \Gamma+\int_{\Gamma_{2}} p_{i j}^{*} \bar{u}_{i} d \Gamma+\int_{\Gamma_{2}} p_{i j}^{*} u_{i} d \Gamma
$$


onde:

$$
\Gamma=\Gamma_{1}+\Gamma_{2}
$$

Como a Identidade Somigliana fornece apenas os deslocamentos para pontos no interior do domínio, há a necessidade de um artifício para torná-la válida também para os pontos situados no contorno.

Uma solução matemática utilizada é ampliar o domínio original $\Omega+\Gamma$, através da adição infinitesimal $\Omega_{\varepsilon}$.

Essa adição é feita de modo a considerar um acréscimo de raio $\varepsilon$, centrado no ponto "s", formando assim um novo contorno $\Gamma-\bar{\Gamma}+\Gamma_{\varepsilon}$ e um novo domínio $\Omega+\Omega_{\varepsilon}$, fazendo com que o ponto "s" passe a pertencer ao domínio, como mostra a figura abaixo:
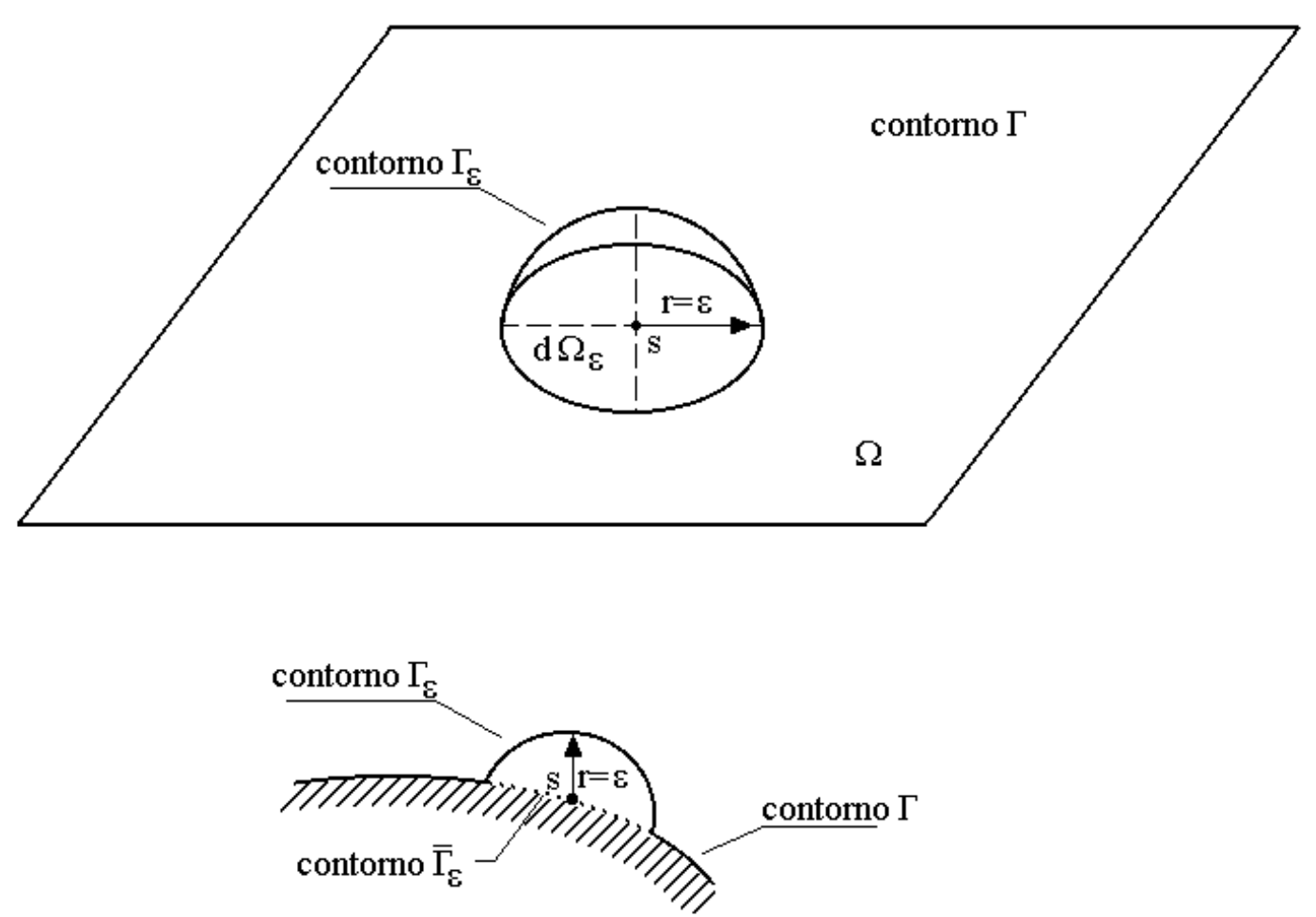

Figura 2.4 - Acréscimo do domínio $\Omega$ 
Assim, pode-se escrever a equação (2.34):

$$
u_{i}=-\int_{\Gamma-\bar{\Gamma}} p_{i j}^{*} u_{j} d \Gamma+\int_{\Gamma-\bar{\Gamma}} u_{i j}^{*} p_{j} d \Gamma+\int_{\Omega} u_{i j}^{*} b_{j} d \Omega \int_{\Gamma_{\varepsilon}} p_{i j}^{*} u_{j} d \Gamma+\int_{\Gamma_{\varepsilon}} u_{i j}^{*} p_{j} d \Gamma+\int_{\Omega_{\varepsilon}} u_{i j}^{*} b_{j} d \Omega
$$

Retomando ao domínio inicial, isto é, fazendo-se $\varepsilon \rightarrow 0$, temos $\Omega_{\varepsilon} \rightarrow 0$. Assim:

$$
\mathrm{u}_{\mathrm{i}}=\lim _{\varepsilon \rightarrow 0}\left[-\int_{\Gamma-\bar{\Gamma}} \mathrm{p}_{\mathrm{ij}}^{*} \mathrm{u}_{\mathrm{j}} \mathrm{d} \Gamma+\int_{\Gamma-\bar{\Gamma}} \mathrm{u}_{\mathrm{ij}}^{*} \mathrm{p}_{\mathrm{j}} \mathrm{d} \Gamma+\int_{\Omega} \mathrm{u}_{\mathrm{ij}}^{*} \mathrm{~b}_{\mathrm{j}} \mathrm{d} \Omega \int_{\Gamma_{\varepsilon}} \mathrm{p}_{\mathrm{ij}}^{*} \mathrm{u}_{\mathrm{j}} \mathrm{d} \Gamma+\int_{\Gamma_{\varepsilon}} \mathrm{u}_{\mathrm{ij}}^{*} \mathrm{p}_{\mathrm{j}} \mathrm{d} \Gamma+\int_{\Omega_{\varepsilon}} \mathrm{u}_{\mathrm{ij}}^{*} \mathrm{~b}_{\mathrm{j}} \mathrm{d} \Omega\right]
$$

Analisando separadamente cada termo da equação acima, nota-se que:

$$
\begin{aligned}
& \left.\lim _{\varepsilon \rightarrow \mathrm{o}} \mid \int_{\Gamma-\bar{\Gamma}} \mathrm{p}_{\mathrm{ij}}^{*} \mathrm{u}_{\mathrm{j}} \mathrm{d} \Gamma\right\rfloor=\int_{\Gamma} \mathrm{p}_{\mathrm{ij}}^{*} \mathrm{u}_{\mathrm{j}} \mathrm{d} \Gamma \\
& \left.\lim _{\varepsilon \rightarrow \mathrm{o}} \mid \int_{\Gamma-\bar{\Gamma}} \mathrm{u}_{\mathrm{ij}}^{*} \mathrm{p}_{\mathrm{j}} \mathrm{d} \Gamma\right\rfloor=\int_{\Gamma} \mathrm{u}_{\mathrm{ij}}^{*} \mathrm{p}_{\mathrm{j}} \mathrm{d} \Gamma
\end{aligned}
$$

Restando agora, a análise das quatro últimas parcelas da equação (2.37)

Quanto as parcelas referentes ao domínio, ou seja, a $3^{\underline{a}}$ e $6^{\mathrm{a}}$ verifica-se que fazendo $\varepsilon \rightarrow 0$, a integral de $\Omega$ representa o domínio todo do problema, enquanto $\Omega_{\varepsilon}$ tende a zero, ou seja:

$$
\lim _{\varepsilon \rightarrow 0}\left[\int_{\Omega+\Omega_{\varepsilon}} u_{i j}^{*} b_{j} d \Omega\right]=\int_{\Omega} u_{i j}^{*} b_{j} d \Omega
$$

Em relação a quinta parcela da equação (2.37), verifica-se que os valores de $\mathrm{u}_{\mathrm{ij}}$ são da ordem de $1 / \varepsilon$, enquanto a integração no contorno $\Gamma_{\varepsilon}$ produz termos da ordem de $\varepsilon^{2}$, podendo concluir que:

$$
\lim _{\varepsilon \rightarrow 0}\left[\int_{\Gamma_{\varepsilon}} u_{i j}^{*} p_{j} d \Gamma\right]=0
$$

Finalmente, $4^{\mathrm{a}}$ parcela apresenta forte singularidade, ao contrário das demais parcelas. Esta singularidade provoca uma descontinuidade na função $\mathrm{p}_{\mathrm{ij}}^{*}$ no contorno. A integração dos valores de $\mathrm{p}_{\mathrm{ij}}^{*}$ no contorno $\Gamma_{\varepsilon}$ produz termos da ordem de $1 / \varepsilon^{2}$, 
enquanto os termos da integração sobre a superfície são de ordem $\varepsilon^{2}$. Portanto a integral para $\varepsilon \rightarrow 0$ produz um termo independente. Admitindo-se que a parcela da equação (2.37) em questão,satisfaz a equação de Holder e em termos de forças, considerando que o ponto "s" pertença a um contorno sem angulosidade (smooth), tem-se que:

$$
\lim _{\varepsilon \rightarrow 0}\left[\int_{\Gamma_{\varepsilon}} p_{i j}^{*} u_{j} d \Gamma\right]=u_{j} \lim _{\varepsilon \rightarrow 0}\left[\int_{\Gamma_{\varepsilon}} p_{i j}^{*} d \Gamma\right]=\delta_{i j} u_{j}
$$

Assim, a equação (2.37) pode-se escrita da seguinte forma:

$$
\mathrm{C}_{\mathrm{ij}} \mathrm{u}_{\mathrm{i}}=-\int_{\Gamma} \mathrm{p}_{\mathrm{ij}}^{*} \mathrm{u}_{\mathrm{j}} \mathrm{d} \Gamma+\int_{\Gamma} \mathrm{u}_{\mathrm{ij}}^{*} \mathrm{p}_{\mathrm{j}} \mathrm{d} \Gamma+\int_{\Omega} \mathrm{u}_{\mathrm{ij}}^{*} \mathrm{~b}_{\mathrm{j}} \mathrm{d} \Omega
$$

que é conhecida como expressão geral para pontos de domínio e para pontos de contorno.

O termo $\mathrm{C}_{\mathrm{ij}}(\mathrm{s})$ pode assumir diferentes valores, dependendo da sua posição e do tipo de solução fundamental utilizada:

Solução de Kelvin ou Mindlin

$$
\begin{aligned}
& \mathrm{s} \in \Omega \longrightarrow \mathrm{C}_{\mathrm{ij}}(\mathrm{s})=\delta \mathrm{ij} \\
& \mathrm{s} \in \Gamma \longrightarrow \mathrm{C}_{\mathrm{ij}}(\mathrm{s})=\frac{1}{2} \delta \mathrm{ij}
\end{aligned}
$$

Solução de Boussinesq-Cerruti (para "s” na superfície)

$$
\begin{aligned}
& \mathrm{s} \in \Omega \longrightarrow \mathrm{C}_{\mathrm{ij}}(\mathrm{s})=\delta \mathrm{ij} \\
& \mathrm{s} \in \Gamma \longrightarrow \mathrm{C}_{\mathrm{ij}}(\mathrm{s})=\delta \mathrm{ij}
\end{aligned}
$$


Por praticidade, representa-se $\delta_{\mathrm{ij}}$ na forma matricial:

$$
\delta_{\mathrm{ij}}=[\mathrm{I}]=\left[\begin{array}{lll}
1 & 0 & 0 \\
0 & 1 & 0 \\
0 & 0 & 1
\end{array}\right]
$$




\section{SISTEMA ESTACA-SOLO}

\subsection{INTRODUÇÃO}

Neste capitulo serão apresentadas as formulações para o conjunto estaca-solo, suas discretizações e hipóteses admitidas. Na seqüência, apresentam-se as etapas algébricas da obtenção das matrizes que compõem o sistema final MEC/MEF, bem com o seu acoplamento.

\subsection{HIPÓTESES BÁSICAS}

O comportamento do sistema estaca-solo é um assunto muito complexo, envolvendo muitas variáveis, sendo assim necessário adotar algumas hipóteses básicas para a simplificação da análise numérica. Dentre essas simplificações, podem ser citadas:

- O solo e as estacas estão livres de tensões iniciais, decorrentes da instalação das mesmas;

- Admite-se que tanto o solo como as estacas trabalham no regime elásticolinear; 
- As estacas estão totalmente imersas num em um semi-espaço, elástico linear, isótropo e homogêneo.

- Admite-se que a estaca possui superficie rugosa, não existindo deslocamento relativo entre a mesma e o solo;

- A distância entre as estacas são tomadas eixo a eixo;

- As forças volumétricas são desprezadas.

\subsection{ESTACA ISOLADA}

Nesta etapa descreve-se toda formulação para o caso de uma estaca isolada, estendendo-se mais adiante, para o caso geral de uma estaca inclinada e grupo de estacas.

Todo o equacionamento, bem como a escolha das funções interpoladoras para deslocamentos e forças de superfície, foram baseadas no trabalho desenvolvido por MATOS FILHO.

Discretizando a estaca como um elemento de barra, com quatro nós eqüidistantes, um em cada extremidade e outros dois ao longo do seu comprimento, MATOS FILHO, através de sucessivas tentativas utilizando várias funções polinomiais para representar os deslocamentos e forças de interação, chegou a um elemento final considerado eficiente, composto por 14 parâmetros nodais, sendo quatro deslocamentos lineares em cada uma das direções $\left(\mathrm{X}_{1}, \mathrm{X}_{2}\right.$ e $\left.\mathrm{X}_{3}\right)$ e mais dois parâmetros localizados na cabeça da estaca, referentes às rotações em torno dos eixos $\mathrm{X}_{1} \mathrm{e} \mathrm{X}_{2}$. Os deslocamentos transversais ao longo da estaca foram aproximados por um polinômio de $4^{\circ}$ grau e os deslocamentos axiais foram representados por uma função polinomial cúbica. As forças de interação normais a estaca foram representadas por polinômios de $3^{\circ}$ grau, e a força cisalhante que ocorrem ao longo 
do fuste foi admitida como um polinômio quadrático. A tensão normal que ocorre na base da estaca é admitida constante.

\subsubsection{Estaca vertical isolada}

Considerando a estaca com um único elemento finito com deslocamentos transversais aproximados por uma função polinomial de $4^{\mathrm{a}}$ ordem e deslocamentos axiais aproximados por um polinômio cúbico, discretizado por 4 nós, conforme a figura abaixo:

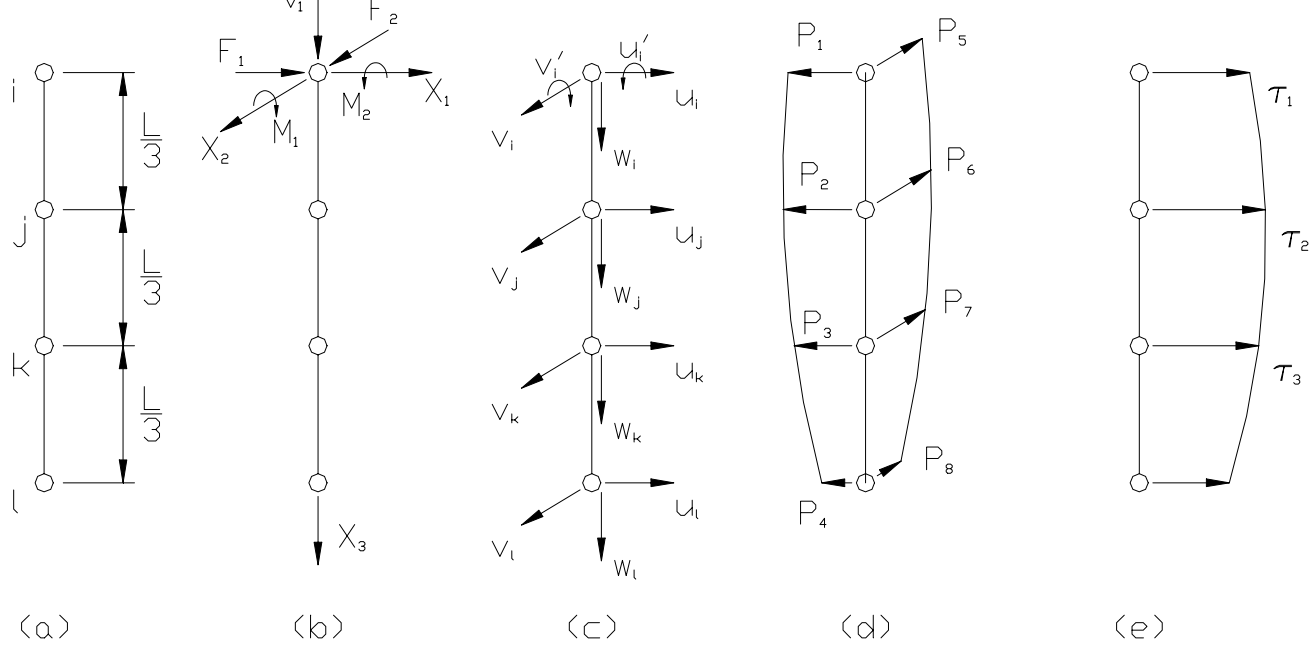

Figura 3.1 - (a) Discretização do elemento; (b) Forças no topo do elemento; (c) Parâmetros nodais de deslocamento; (d) Forças de interação nas direções $\mathrm{X}_{1}$ e $\mathrm{X}_{2}$; (e) Forças de interação na direção $\mathrm{X}_{3}$ 
Assim para a direção $\mathrm{X}_{1}$, temos:

$$
\begin{aligned}
& u_{a p}(z)=A_{1} z^{4}+B_{1} z^{3}+C_{1} z^{2}+D_{1} z+E_{1} \\
& u^{\prime}{ }_{\text {ap }}(z)=4 A_{1} z^{3}+3 B_{1} z^{2}+2 C_{1} z+D_{1} \\
& u^{\prime \prime}{ }_{\text {ap }}(z)=12 A_{1} z^{2}+6 B_{1} z+2 C_{1} \\
& E \\
& P_{1}(z)=A_{2} z^{3}+B_{2} z^{2}+C_{2} z+D_{2}
\end{aligned}
$$

Para a direção $\mathrm{X}_{2}$ :

$$
\begin{aligned}
& \mathrm{v}_{\text {ap }}(\mathrm{z})=\mathrm{A}_{3} \mathrm{z}^{4}+\mathrm{B}_{3} \mathrm{z}^{3}+\mathrm{C}_{3} \mathrm{z}^{2}+\mathrm{D}_{3} \mathrm{z}+\mathrm{E}_{3} \\
& \mathrm{v}_{\text {ap }}(\mathrm{z})=4 \mathrm{~A}_{3} \mathrm{z}^{3}+3 \mathrm{~B}_{3} \mathrm{z}^{2}+2 \mathrm{C}_{3} \mathrm{z}+\mathrm{D}_{3} \\
& \mathrm{v}_{\text {ap }}(\mathrm{z})=12 \mathrm{~A}_{3} \mathrm{z}^{2}+6 \mathrm{~B}_{3} \mathrm{z}+2 \mathrm{C}_{3} \\
& \mathrm{E} \\
& \mathrm{Px}_{2}(\mathrm{z})=\mathrm{A}_{4} \mathrm{z}^{3}+\mathrm{B}_{4} \mathrm{z}^{2}+\mathrm{C}_{4} \mathrm{z}+\mathrm{D}_{4}
\end{aligned}
$$

E para a direção $\mathrm{X}_{3}$ :

$$
\begin{aligned}
& \mathrm{W}_{\text {ap }}(\mathrm{z})=\mathrm{A}_{5} \mathrm{z}^{3}+\mathrm{B}_{5} \mathrm{z}^{2}+\mathrm{C}_{5} \mathrm{z}+\mathrm{D}_{5} \\
& \mathrm{w}^{\prime}{ }_{\text {ap }}(\mathrm{z})=3 \mathrm{~A}_{5} \mathrm{z}^{2}+2 \mathrm{~B}_{5} \mathrm{z}+\mathrm{C}_{5} \\
& \mathrm{E} \\
& \tau_{\mathrm{p}}(\mathrm{z})=\mathrm{A}_{6} \mathrm{z}^{2}+\mathrm{B}_{6} \mathrm{z}+\mathrm{C}_{6} \\
& \tau_{\mathrm{b}}(\mathrm{z})=1
\end{aligned}
$$

Substituindo valores para os nós “i”, “j”, "k" e "l” temos:

$$
\left(\begin{array}{ccccc}
0 & 0 & 0 & 0 & 1 \\
0 & 0 & 0 & 1 & 0 \\
\frac{\mathrm{L}^{4}}{81} & \frac{\mathrm{L}^{3}}{27} & \frac{\mathrm{L}^{2}}{9} & \frac{\mathrm{L}}{3} & 1 \\
\frac{16 \mathrm{~L}^{4}}{81} & \frac{8 \mathrm{~L}^{3}}{27} & \frac{4 \mathrm{~L}^{2}}{9} & \frac{2 \mathrm{~L}}{3} & 1 \\
\mathrm{~L}^{4} & \mathrm{~L}^{3} & \mathrm{~L}^{2} & \mathrm{~L} & 1
\end{array}\right) \cdot\left(\begin{array}{c}
\mathrm{A}_{1} \\
\mathrm{~B}_{1} \\
\mathrm{C}_{1} \\
\mathrm{D}_{1} \\
\mathrm{E}_{1}
\end{array}\right)=\left(\begin{array}{c}
\mathrm{u}_{\mathrm{i}} \\
\mathrm{u}_{\mathrm{i}} \\
\mathrm{u}_{\mathrm{j}} \\
\mathrm{u}_{\mathrm{k}} \\
\mathrm{u}_{\mathrm{l}}
\end{array}\right)
$$




$$
\begin{aligned}
& \left(\begin{array}{ccccc}
0 & 0 & 0 & 0 & 1 \\
0 & 0 & 0 & 1 & 0 \\
\frac{\mathrm{L}^{4}}{81} & \frac{\mathrm{L}^{3}}{27} & \frac{\mathrm{L}^{2}}{9} & \frac{\mathrm{L}}{3} & 1 \\
\frac{16 \mathrm{~L}^{4}}{81} & \frac{8 \mathrm{~L}^{3}}{27} & \frac{4 \mathrm{~L}^{2}}{9} & \frac{2 \mathrm{~L}}{3} & 1 \\
\mathrm{~L}^{4} & \mathrm{~L}^{3} & \mathrm{~L}^{2} & \mathrm{~L} & 1
\end{array}\right) \cdot\left(\begin{array}{c}
\mathrm{A}_{3} \\
\mathrm{~B}_{3} \\
\mathrm{C}_{3} \\
\mathrm{D}_{3} \\
\mathrm{E}_{3}
\end{array}\right)=\left(\begin{array}{c}
\mathrm{v}_{\mathrm{i}} \\
\mathrm{v}_{\mathrm{i}} \\
\mathrm{v}_{\mathrm{j}} \\
\mathrm{v}_{\mathrm{k}} \\
\mathrm{v}_{1}
\end{array}\right) \\
& \left(\begin{array}{cccc}
0 & 0 & 0 & 1 \\
\frac{\mathrm{L}^{3}}{27} & \frac{\mathrm{L}^{2}}{9} & \frac{\mathrm{L}}{3} & 1 \\
\frac{8 \mathrm{~L}^{3}}{27} & \frac{4 \mathrm{~L}^{2}}{9} & \frac{2 \mathrm{~L}}{3} & 1 \\
\mathrm{~L}^{3} & \mathrm{~L}^{2} & \mathrm{~L} & 1
\end{array}\right) \cdot\left(\begin{array}{l}
\mathrm{A}_{5} \\
\mathrm{~B}_{5} \\
\mathrm{C}_{5} \\
\mathrm{D}_{5}
\end{array}\right)=\left(\begin{array}{c}
\mathrm{w}_{\mathrm{i}} \\
\mathrm{w}_{\mathrm{j}} \\
\mathrm{w}_{\mathrm{k}} \\
\mathrm{w}_{1}
\end{array}\right)
\end{aligned}
$$

Pode-se escrever cada uma das expressões acima da seguinte maneira:

$$
[C]\{\alpha\}=\left\{\delta_{\mathrm{e}}\right\}
$$

Isolando-se o termo $\{\alpha\}$, tem-se:

$$
\{\alpha\}=[C]^{-1}\left\{\delta_{\mathrm{e}}\right\}
$$

Lembrando que a equações (3.1a), (3.2a) e (3.3c) pode ser escrita na forma matricial:

$$
\begin{aligned}
& u_{\text {ap }}(z)=\left\{\begin{array}{lllll}
z^{4} & z^{3} & z^{2} & z & 1
\end{array}\right\}\left\{\begin{array}{l}
A_{1} \\
B_{1} \\
C_{1} \\
D_{1} \\
E_{1}
\end{array}\right\} \\
& v_{\text {ap }}(z)=\left\{\begin{array}{lllll}
z^{4} & z^{3} & z^{2} & z & 1
\end{array}\right\}\left\{\begin{array}{l}
A_{3} \\
B_{3} \\
C_{3} \\
D_{3} \\
E_{3}
\end{array}\right\}
\end{aligned}
$$




$$
\mathrm{w}_{\text {ap }}(\mathrm{z})=\left\{\begin{array}{llll}
\mathrm{z}^{3} & \mathrm{z}^{2} & \mathrm{z} & 1
\end{array}\right\}\left\{\begin{array}{l}
\mathrm{A}_{5} \\
\mathrm{~B}_{5} \\
\mathrm{C}_{5} \\
\mathrm{D}_{5}
\end{array}\right\}
$$

Substituindo (3.6) em (3.7), temos

$$
\mathrm{u}_{\mathrm{ap}}(\mathrm{z})=\{\phi\}^{\mathrm{T}}[\mathrm{C}]^{-1}\left\{\delta_{\mathrm{e}}\right\}
$$

Derivando as expressões:

$$
\begin{aligned}
& \mathrm{u}_{\mathrm{ap},}(\mathrm{z})=\{\phi\}^{\mathrm{T}}[\mathrm{C}]^{-1}\left\{\delta_{\mathrm{e}}\right\} \\
& \mathrm{v}_{\mathrm{ap}},(\mathrm{z})=\left\{\phi^{\prime}\right\}^{\mathrm{T}}[\mathrm{C}]^{-1}\left\{\delta_{\mathrm{e}}\right\} \\
& \mathrm{w}_{\mathrm{ap}},(\mathrm{z})=\{\phi\}^{\mathrm{T}}[\mathrm{C}]^{-1}\left\{\delta_{\mathrm{e}}\right\}
\end{aligned}
$$

Agora, considerando as forças de interface nas direções $X_{1}$ e $X_{2}$ aproximadas por um polinômio cúbico, e na direção $X_{3}$ por uma função polinomial quadrática, temos:

$$
\begin{aligned}
& P_{1}(z)=A_{2} z^{3}+B_{2} z^{2}+C_{2} z+D_{2} \\
& P_{2}(z)=A_{4} z^{3}+B_{4} z^{2}+C_{4} z+D_{4} \\
& P_{3}(z)=A_{6} z^{2}+B_{6} z+C_{6} \\
& \tau_{b}(z)=1
\end{aligned}
$$

Pode-se escrever estes polinômios como uma soma de funções de forma multiplicados pelos respecitvos valores das forças de interface nos pontos de colocação, ou seja:

$$
\begin{aligned}
& \mathrm{Px}_{1}(\mathrm{z})=\phi_{1} \mathrm{P}_{1}+\phi_{2} \mathrm{P}_{2}+\phi_{3} \mathrm{P}_{3}+\phi_{4} \mathrm{P}_{4} \\
& \mathrm{Px}_{2}(\mathrm{z})=\phi_{1} \mathrm{P}_{5}+\phi_{2} \mathrm{P}_{6}+\phi_{3} \mathrm{P}_{7}+\phi_{4} \mathrm{P}_{8} \\
& \mathrm{Px}_{3}(\mathrm{z})=\varphi_{1} \tau_{1}+\varphi_{2} \tau_{2}+\varphi_{3} \tau_{3} \\
& \mathrm{ou} \\
& \mathrm{Px}_{1}=\{\bar{\phi}\}^{\mathrm{T}}\{\mathrm{P}\} \\
& \mathrm{Px}_{2}=\{\bar{\phi}\}^{\mathrm{T}}\{\mathrm{P}\} \\
& \mathrm{Px}_{3}=\left\{(-)^{\mathrm{T}}\{\mathrm{P}\}\right.
\end{aligned}
$$


onde:

$$
\begin{gathered}
\text { Direção } \mathrm{X}_{1} \text { e } \mathrm{X}_{2} \\
\{\bar{\phi}\}=\left\{\begin{array}{l}
\bar{\phi}_{1} \\
\bar{\phi}_{2} \\
\bar{\phi}_{3} \\
\bar{\phi}_{4}
\end{array}\right\}=\left\{\begin{array}{c}
\frac{-9 \mathrm{z}^{3}}{2 \mathrm{~L}^{3}}+\frac{9 \mathrm{z}^{2}}{\mathrm{~L}^{2}}-\frac{11 \mathrm{z}}{2 \mathrm{~L}}+1 \\
\frac{27 \mathrm{z}^{3}}{2 \mathrm{~L}^{3}}-\frac{45 \mathrm{z}^{2}}{2 \mathrm{~L}^{2}}+\frac{9 \mathrm{z}}{\mathrm{L}} \\
\frac{-27 \mathrm{z}^{3}}{2 \mathrm{~L}^{3}}+\frac{18 \mathrm{z}^{2}}{\mathrm{~L}^{2}}-\frac{9 \mathrm{z}}{2 \mathrm{~L}} \\
\frac{9 \mathrm{z}^{3}}{2 \mathrm{~L}^{3}}-\frac{9 \mathrm{z}^{2}}{2 \mathrm{~L}^{2}}+\frac{\mathrm{z}}{\mathrm{L}}
\end{array}\right\} \quad\{\varphi\}=\left\{\begin{array}{l}
\bar{\varphi}_{1} \\
\frac{\varphi_{2}}{\varphi_{3}}
\end{array}\right\}=\left\{\begin{array}{l}
\frac{9 \mathrm{z}^{2}}{2 \mathrm{~L}^{2}}-\frac{9 \mathrm{z}}{2 \mathrm{~L}}+1 \\
\frac{-9 \mathrm{z}^{2}}{\mathrm{~L}^{2}}+\frac{6 \mathrm{z}}{\mathrm{L}} \\
\frac{9 \mathrm{z}^{2}}{2 \mathrm{~L}^{2}}-\frac{3 \mathrm{z}}{2 \mathrm{~L}}
\end{array}\right\}
\end{gathered}
$$

Fazendo $\xi=\mathrm{z} / \mathrm{L}$, temos

$$
\begin{aligned}
& \text { Direção } X_{1} \text { e } X_{2} \\
& \text { Direção } X_{3} \\
& \left.\{-\bar{\phi}\}=\left\{\begin{array}{c}
-\frac{9}{2} \xi^{3}+9 \xi^{2}-\frac{11}{2} \xi+1 \\
\frac{27}{2} \xi^{3}-\frac{45}{2} \xi^{2}+9 \xi \\
-\frac{27}{2} \xi^{3}+18 \xi^{2}-\frac{9}{2} \xi \\
\frac{9}{2} \xi^{3}-\frac{9}{2} \xi^{2}+\xi
\end{array}\right\} \quad\{-)\right\}=\left\{\begin{array}{c}
\bar{\varphi}_{1} \\
\bar{\varphi}_{2} \\
\varphi_{3}
\end{array}\right\}=\left\{\begin{array}{c}
\frac{9}{2} \xi^{2}-\frac{9}{2} \xi+1 \\
-9 \xi^{2}+6 \xi \\
\frac{9}{2} \xi^{2}-\frac{3}{2} \xi
\end{array}\right\}
\end{aligned}
$$

onde:

$\xi$ é a cota adimensional;

z é a cota do ponto em questão;

L é o comprimento da estaca.

Sabe-se que a energia potencial total é dada pela soma da parcela referente a energia de deformação e pela energia potencial do carregamento externo.

$$
\Pi=\mathrm{U}+\Omega
$$


onde:

$\Pi$ : energia potencial total;

U: energia potencial de deformação;

$\Omega$ : energia potencial das cargas externas.

Assim, pode-se escrever o funcional para o elemento de estaca:

$$
\begin{aligned}
\Pi= & \frac{\mathrm{E}_{\mathrm{P}} \mathrm{I}_{\mathrm{P}}}{2} \int_{0}^{\mathrm{L}} \mathrm{u}^{\prime \prime}{ }_{\text {ap }}(\mathrm{z})^{2} \mathrm{dz}+\frac{\mathrm{E}_{\mathrm{P}} \mathrm{I}_{\mathrm{P}}}{2} \int_{0}^{\mathrm{L}} \mathrm{v}^{\prime \prime}{ }_{\text {ap }}(\mathrm{z})^{2} \mathrm{dz}+\frac{\mathrm{E}_{\mathrm{P}} \mathrm{A}_{\mathrm{P}}}{2} \int_{0}^{\mathrm{L}} \mathrm{w}_{\text {ap }}^{\prime}(\mathrm{z})^{2} \mathrm{dz}+ \\
& +\int_{0}^{\mathrm{L}} \mathrm{Px}_{1}(\mathrm{z}) \mathrm{u}_{\mathrm{ap}}(\mathrm{z}) \mathrm{dz}+\int_{0}^{\mathrm{L}} \mathrm{Px}_{2}(\mathrm{z}) \mathrm{v}_{\mathrm{ap}}(\mathrm{z}) \mathrm{dz}+\int_{0}^{\mathrm{L}} \mathrm{Px}_{3}(\mathrm{z}) \mathrm{w}_{\mathrm{ap}}(\mathrm{z}) \mathrm{dz}+ \\
& +\int_{\mathrm{A}_{\mathrm{p}}} \sigma_{\mathrm{b}} \mathrm{w}_{1} \mathrm{dA}_{\mathrm{p}}-\mathrm{F}_{1} \mathrm{u}_{\mathrm{i}}-\mathrm{F}_{2} \mathrm{v}_{\mathrm{i}}-\mathrm{M}_{2} \mathrm{u}_{\mathrm{i}}^{\prime}-\mathrm{M}_{1} \mathrm{v}_{\mathrm{i}}^{\prime}
\end{aligned}
$$

onde:

$\mathrm{E}_{\mathrm{p}}$ é o módulo de elasticidade longitudinal da estaca;

$\mathrm{I}_{\mathrm{p}}$ é o momento de inércia da estaca;

$\mathrm{A}_{\mathrm{p}}$ é a área da seção transversal da estaca.

$F_{i}$ é a força externa aplicada na direção "i”,

$\mathrm{M}_{\mathrm{i}}$ é o momento externo aplicado em torno da direção " $\mathrm{i}$ ”.

Pode-se dividir o funcional dado em (3.15) em uma soma de funcionais referentes às energias dadas em cada direção:

$$
\Pi_{\mathrm{ap}}=\Pi_{1}+\Pi_{2}+\Pi_{3}
$$


onde:

$$
\begin{aligned}
& \Pi_{1}=\frac{\mathrm{E}_{\mathrm{P}} \mathrm{I}_{\mathrm{P}}}{2} \int_{0}^{\mathrm{L}} \mathrm{u}^{\prime \prime}{ }_{\text {ap }}(\mathrm{z})^{2} \mathrm{dz}+\int_{0}^{\mathrm{L}} \operatorname{Px}_{1}(\mathrm{z}) \mathrm{u}_{\mathrm{ap}}(\mathrm{z}) \mathrm{dz}-\mathrm{F}_{1} \mathrm{u}_{\mathrm{i}}-\mathrm{M}_{1} \mathrm{u}_{\mathrm{i}}^{\prime} \\
& \Pi_{2}=\frac{\mathrm{E}_{\mathrm{P}} \mathrm{I}_{\mathrm{P}}}{2} \int_{0}^{\mathrm{L}} \mathrm{v}^{\prime \prime}{ }_{\text {ap }}(\mathrm{z})^{2} \mathrm{dz}+\int_{0}^{\mathrm{L}} \operatorname{Px}_{2}(\mathrm{z}) \mathrm{v}_{\text {ap }}(\mathrm{z}) \mathrm{dz}-\mathrm{F}_{2} \mathrm{v}_{\mathrm{i}}-\mathrm{M}_{2} \mathrm{v}_{\mathrm{i}}^{\prime} \\
& \Pi_{3}=\frac{\mathrm{E}_{\mathrm{P}} \mathrm{A}_{\mathrm{P}}}{2} \int_{0}^{\mathrm{L}} \mathrm{w}_{\text {ap }}^{\prime}(\mathrm{z})^{2} \mathrm{dz}+\int_{0}^{\mathrm{L}} \operatorname{Px}_{3}(\mathrm{z}) \mathrm{w}_{\text {ap }}(\mathrm{z}) \mathrm{dz}-\mathrm{F}_{3} \mathrm{w}_{\mathrm{i}}+\int_{\mathrm{A}_{\mathrm{p}}} \sigma_{\mathrm{b}} \mathrm{w}_{1} \mathrm{dA}_{\mathrm{p}}
\end{aligned}
$$

Trabalhando agora apenas com a direção $\mathrm{X}_{1}$ e sabendo-se que:

$$
\mathrm{u}_{\mathrm{ap}}(\mathrm{z})^{\prime \prime 2}=\left\{\delta_{\mathrm{e}}\right\}^{\mathrm{T}}[\mathrm{C}]^{-\mathrm{T}}\left\{\phi^{\prime \prime}\right\}\left\{\phi^{\prime \prime}\right\}^{\mathrm{T}}[\mathrm{C}]^{-1}\left\{\delta_{\mathrm{e}}\right\}
$$

Substituindo (3.14) em (3.13) e agrupando os termos,

$$
\Pi_{1}=\frac{\mathrm{E}_{\mathrm{P}} \mathrm{I}_{\mathrm{P}}}{2} \int_{0}^{\mathrm{L}}\left\{\delta_{\mathrm{e}}\right\}^{\mathrm{T}}[\mathrm{C}]^{-\mathrm{T}}\left\{\phi^{\prime \prime}\right\}\left\{\phi^{\prime \prime}\right\}^{\mathrm{T}}[\mathrm{C}]^{-1}\left\{\delta_{\mathrm{e}}\right\} \mathrm{dz}+\int_{0}^{\mathrm{L}}\left\{\delta_{\mathrm{e}}\right\}^{\mathrm{T}}[\mathrm{C}]^{-\mathrm{T}}\{\phi\}\{\bar{\phi}\}^{\mathrm{T}}\{\mathrm{P}\} \mathrm{dz}-\left\{\delta_{\mathrm{e}}\right\}^{\mathrm{T}}
$$

Fazendo:

$$
\begin{aligned}
& {\left[\mathrm{K}_{1}\right]=\frac{\mathrm{E}_{\mathrm{P}} \mathrm{I}_{\mathrm{P}}}{2}[\mathrm{C}]^{-\mathrm{T}} \int_{0}^{\mathrm{L}}\left\{\phi^{\prime \prime}\right\}\left\{\phi^{\prime \prime}\right\}^{\mathrm{T}} \mathrm{dz}[\mathrm{C}]^{-1}} \\
& {\left[\mathrm{Q}_{1}\right]=[\mathrm{C}]^{-\mathrm{T}} \int_{0}^{\mathrm{L}}\{\phi\}\{\bar{\phi}\}^{\mathrm{T}} \mathrm{dz}}
\end{aligned}
$$

Obtem-se

$$
\Pi_{1}=\left\{\delta_{\mathrm{e}}\right\}^{\mathrm{T}}\left[\mathrm{K}_{1}\right]\left\{\delta_{\mathrm{e}}\right\}+\left\{\delta_{\mathrm{e}}\right\}^{\mathrm{T}}\left[\mathrm{Q}_{1}\right]\{\mathrm{P}\}-\left\{\delta_{\mathrm{e}}\right\}^{\mathrm{T}}\left\{\mathrm{F}_{1}\right\}
$$

Minimizando-se o funcional, ou seja, derivando-se a equação (3.20) em função dos parâmetros nodais, obtêm-se:

$$
\left[\mathrm{K}_{1}\right]\left\{\delta_{\mathrm{e}}\right\}=\left\{\mathrm{F}_{1}\right\}-\left[\mathrm{Q}_{1}\right]\left\{\mathrm{P}_{\mathrm{p}}\right\}
$$


onde:

$$
\begin{aligned}
& {\left[\mathrm{K}_{1}\right]=\frac{\mathrm{E}_{\mathrm{P}} \mathrm{I}_{\mathrm{P}}}{40 \mathrm{~L}^{3}}\left[\begin{array}{ccccc}
23722 & 4084 \mathrm{~L} & -42876 & 26838 & -7684 \\
4084 \mathrm{~L} & 808 \mathrm{~L}^{2} & -6912 \mathrm{~L} & 3996 \mathrm{~L} & -1168 \mathrm{~L} \\
-42876 & -6912 \mathrm{~L} & 81648 & -55404 & 16632 \\
26838 & 3996 \mathrm{~L} & -55404 & 42282 & -13716 \\
-7684 & -1168 \mathrm{~L} & 16632 & -13716 & 4758
\end{array}\right]} \\
& {\left[\mathrm{Q}_{1}\right]=\frac{\mathrm{L}}{6720}\left[\begin{array}{cccc}
721 & 495 & -45 & 285 \\
38 \mathrm{~L} & 18 \mathrm{~L} & 18 \mathrm{~L} & 38 \mathrm{~L} \\
54 & 2430 & -486 & -486 \\
27 & -243 & 2673 & 567 \\
38 & -162 & 378 & 474
\end{array}\right]}
\end{aligned}
$$

$\mathrm{Na}$ direção $\mathrm{X}_{2}$, como os polinômios adotados para representar os deslocamentos e forças de interação são iguais aos da direção $X_{1}$, bem como os carregamentos externos, as matrizes $\left[\mathrm{K}_{1}\right]$ e $\left[\mathrm{Q}_{1}\right]$ obtidas no processo descrito acima, logo também serão iguais à $\left[\mathrm{K}_{2}\right]$ e $\left[\mathrm{Q}_{2}\right]$ respectivamente. Já na direção $\mathrm{X}_{3}$, a parcela do funcional é dada por (3.17c):

$$
\Pi_{3}=\frac{\mathrm{E}_{\mathrm{P}} \mathrm{A}_{\mathrm{P}}}{2} \int_{0}^{\mathrm{L}} \mathrm{w}^{\prime}{ }_{\text {ap }}(\mathrm{z})^{2} \mathrm{dz}+\int_{0}^{\mathrm{L}} \mathrm{Px}_{3}(\mathrm{z}) \mathrm{w}_{\text {ap }}(\mathrm{z}) \mathrm{dz}-\mathrm{F}_{3} \mathrm{w}_{\mathrm{i}}+\int_{\mathrm{A}_{\mathrm{p}}} \sigma_{\mathrm{b}} \mathrm{w}_{1} \mathrm{dA} \mathrm{A}_{\mathrm{p}}
$$

Analogamente aos procedimentos executados no item anterior, temos:

$$
\left[\mathrm{K}_{3}\right]\left\{\delta_{\mathrm{e}}\right\}=\left\{\mathrm{F}_{3}\right\}-\left[\mathrm{Q}_{3}\right]\left\{\mathrm{P}_{\mathrm{p}}\right\}
$$

Onde:

$$
\left[\mathrm{K}_{3}\right]=\frac{\mathrm{E}_{\mathrm{I}} \mathrm{A}_{\mathrm{p}}}{40 \mathrm{~L}}\left[\begin{array}{cccc}
148 & -189 & 54 & -13 \\
-189 & 432 & -297 & 54 \\
54 & -297 & 432 & -189 \\
-13 & 54 & -189 & 148
\end{array}\right]
$$




$$
\left[\mathrm{Q}_{3}\right]=\left[\begin{array}{cccc}
\frac{7 \mathrm{~L}}{80} & \frac{\mathrm{L}}{40} & \frac{\mathrm{L}}{80} & 0 \\
\frac{3 \mathrm{~L}}{80} & \frac{9 \mathrm{~L}}{20} & \frac{-9 \mathrm{~L}}{80} & 0 \\
\frac{3 \mathrm{~L}}{80} & \frac{-9 \mathrm{~L}}{40} & \frac{9 \mathrm{~L}}{16} & 0 \\
\frac{7 \mathrm{~L}}{80} & \frac{-\mathrm{L}}{4} & \frac{23 \mathrm{~L}}{80} & 1
\end{array}\right]
$$

Para representar o funcional do sistema todo, é necessário somar as contribuições parciais dos funcionais relativos a cada uma das direções $\mathrm{X}_{1}, \mathrm{X}_{2}$ e $\mathrm{X}_{3}$, e reagrupar as matrizes resultantes, num único sistema final, dado por:

$$
[\mathrm{K}]\left\{\mathrm{U}_{\mathrm{p}}\right\}=\{\mathrm{F}\}-[\mathrm{Q}]\left\{\mathrm{P}_{\mathrm{p}}\right\}
$$

Onde:

$[\mathrm{K}]=\left[\begin{array}{cccccccccccccc}\mathrm{a}_{11} & 0 & 0 & \mathrm{a}_{12} & 0 & \mathrm{a}_{13} & 0 & 0 & \mathrm{a}_{14} & 0 & 0 & \mathrm{a}_{15} & 0 & 0 \\ 0 & \mathrm{a}_{11} & 0 & 0 & -\mathrm{a}_{12} & 0 & \mathrm{a}_{13} & 0 & 0 & \mathrm{a}_{14} & 0 & 0 & \mathrm{a}_{15} & 0 \\ 0 & 0 & \mathrm{c}_{11} & 0 & 0 & 0 & 0 & \mathrm{c}_{12} & 0 & 0 & \mathrm{c}_{13} & 0 & 0 & \mathrm{c}_{14} \\ \mathrm{a}_{21} & 0 & 0 & \mathrm{a}_{22} & 0 & \mathrm{a}_{23} & 0 & 0 & \mathrm{a}_{24} & 0 & 0 & \mathrm{a}_{25} & 0 & 0 \\ 0 & -\mathrm{a}_{21} & 0 & 0 & \mathrm{a}_{22} & 0 & -\mathrm{a}_{23} & 0 & 0 & -\mathrm{a}_{24} & 0 & 0 & -\mathrm{a}_{25} & 0 \\ \mathrm{a}_{31} & 0 & 0 & \mathrm{a}_{32} & 0 & \mathrm{a}_{33} & 0 & 0 & \mathrm{a}_{34} & 0 & 0 & \mathrm{a}_{35} & 0 & 0 \\ 0 & \mathrm{a}_{31} & 0 & 0 & -\mathrm{a}_{32} & 0 & \mathrm{a}_{33} & 0 & 0 & \mathrm{a}_{34} & 0 & 0 & \mathrm{a}_{35} & 0 \\ 0 & 0 & \mathrm{c}_{21} & 0 & 0 & 0 & 0 & \mathrm{c}_{22} & 0 & 0 & \mathrm{c}_{23} & 0 & 0 & \mathrm{a}_{24} \\ \mathrm{a}_{41} & 0 & 0 & \mathrm{a}_{42} & 0 & \mathrm{a}_{43} & 0 & 0 & \mathrm{a}_{44} & 0 & 0 & \mathrm{a}_{54} & 0 & 0 \\ 0 & \mathrm{a}_{41} & 0 & 0 & -\mathrm{a}_{42} & 0 & \mathrm{a}_{43} & 0 & 0 & \mathrm{a}_{44} & 0 & 0 & \mathrm{a}_{45} & 0 \\ 0 & 0 & \mathrm{c}_{31} & 0 & 0 & 0 & 0 & \mathrm{c}_{32} & 0 & 0 & \mathrm{c}_{33} & 0 & 0 & \mathrm{c}_{34} \\ \mathrm{a}_{51} & 0 & 0 & \mathrm{a}_{52} & 0 & \mathrm{a}_{53} & 0 & 0 & \mathrm{a}_{54} & 0 & 0 & \mathrm{a}_{55} & 0 & 0 \\ 0 & \mathrm{a}_{51} & 0 & 0 & -\mathrm{a}_{53} & 0 & \mathrm{a}_{53} & 0 & 0 & \mathrm{a}_{54} & 0 & 0 & \mathrm{a}_{55} & 0 \\ 0 & 0 & \mathrm{c}_{41} & 0 & 0 & 0 & 0 & \mathrm{c}_{42} & 0 & 0 & \mathrm{c}_{43} & 0 & 0 & \mathrm{c}_{44}\end{array}\right]$

$$
\begin{aligned}
& a_{11}=23722 \\
& a_{12}=a_{21}=4084 L \\
& a_{13}=a_{31}=-42876 \\
& a_{14}=a_{41}=26838
\end{aligned}
$$

$$
\begin{aligned}
& a_{25}=a_{52}=-1168 L \\
& a_{33}=81648 \\
& a_{34}=a_{43}=-55404 \\
& a_{35}=a_{53}=16632
\end{aligned}
$$$$
\mathrm{c}_{12}=\mathrm{c}_{21}=-189
$$$$
\mathrm{c}_{13}=\mathrm{c}_{31}=54
$$$$
\mathrm{c}_{14}=\mathrm{c}_{41}=-13
$$$$
\mathrm{c}_{22}=\mathrm{c}_{33}=432
$$ 


$$
\begin{array}{lll}
\mathrm{a}_{15}=\mathrm{a}_{51}=-7684 & \mathrm{a}_{44}=42282 & \mathrm{c}_{23}=\mathrm{c}_{32}=-297 \\
\mathrm{a}_{22}=808 \mathrm{~L}^{2} & \mathrm{a}_{45}=\mathrm{a}_{54}=-13716 & \mathrm{c}_{24}=\mathrm{c}_{42}=54 \\
\mathrm{a}_{23}=\mathrm{a}_{32}=-6912 \mathrm{~L} & \mathrm{a}_{55}=4768 & \mathrm{c}_{34}=\mathrm{c}_{43}=-189 \\
\mathrm{a}_{24}=\mathrm{a}_{42}=3996 \mathrm{~L} & \mathrm{c}_{11}=148 & \mathrm{c}_{44}=148
\end{array}
$$

Lembrando que os termos "a" devem ser multiplicados por $\frac{E_{p} I_{p}}{40 L^{3}}$ e os termos em"c" por $\frac{E_{p} A_{p}}{40 L}$.

$$
[\mathrm{Q}]=\left[\begin{array}{cccccccccccc}
\mathrm{d}_{11} & 0 & 0 & \mathrm{~d}_{12} & 0 & 0 & \mathrm{~d}_{13} & 0 & 0 & \mathrm{~d}_{14} & 0 & 0 \\
0 & \mathrm{~d}_{11} & 0 & 0 & \mathrm{~d}_{12} & 0 & 0 & \mathrm{~d}_{13} & 0 & 0 & \mathrm{~d}_{14} & 0 \\
0 & 0 & \mathrm{e}_{11} & 0 & 0 & \mathrm{e}_{12} & 0 & 0 & \mathrm{e}_{13} & 0 & 0 & \mathrm{e}_{14} \\
\mathrm{~d}_{21} & 0 & 0 & \mathrm{~d}_{22} & 0 & 0 & \mathrm{~d}_{23} & 0 & 0 & \mathrm{~d}_{24} & 0 & 0 \\
0 & -\mathrm{d}_{21} & 0 & 0 & -\mathrm{d}_{22} & 0 & 0 & -\mathrm{d}_{23} & 0 & 0 & -\mathrm{d}_{24} & 0 \\
\mathrm{~d}_{31} & 0 & 0 & \mathrm{~d}_{32} & 0 & 0 & \mathrm{~d}_{33} & 0 & 0 & \mathrm{~d}_{34} & 0 & 0 \\
0 & \mathrm{~d}_{31} & 0 & 0 & \mathrm{~d}_{32} & 0 & 0 & \mathrm{~d}_{33} & 0 & 0 & \mathrm{~d}_{34} & 0 \\
0 & 0 & \mathrm{e}_{21} & 0 & 0 & \mathrm{e}_{22} & 0 & 0 & \mathrm{e}_{23} & 0 & 0 & \mathrm{e}_{24} \\
\mathrm{~d}_{41} & 0 & 0 & \mathrm{~d}_{42} & 0 & 0 & \mathrm{~d}_{43} & 0 & 0 & \mathrm{~d}_{44} & 0 & 0 \\
0 & \mathrm{~d}_{41} & 0 & 0 & \mathrm{~d}_{41} & 0 & 0 & \mathrm{~d}_{43} & 0 & 0 & \mathrm{~d}_{44} & 0 \\
0 & 0 & \mathrm{e}_{31} & 0 & 0 & \mathrm{e}_{32} & 0 & 0 & \mathrm{e}_{23} & 0 & 0 & \mathrm{e}_{34} \\
\mathrm{~d}_{51} & 0 & 0 & \mathrm{~d}_{52} & 0 & 0 & \mathrm{~d}_{53} & 0 & 0 & \mathrm{~d}_{54} & 0 & 0 \\
0 & \mathrm{~d}_{51} & 0 & 0 & \mathrm{~d}_{52} & 0 & 0 & \mathrm{~d}_{53} & 0 & 0 & \mathrm{~d}_{54} & 0 \\
0 & 0 & \mathrm{e}_{41} & 0 & 0 & \mathrm{e}_{42} & 0 & 0 & \mathrm{e}_{43} & 0 & 0 & \mathrm{e}_{44}
\end{array}\right]
$$

$\begin{array}{llll}\mathrm{d}_{11}=721 & \mathrm{~d}_{32}=2430 & \mathrm{~d}_{53}=378 & \mathrm{~d}_{24}=0 \\ \mathrm{~d}_{12}=495 & \mathrm{~d}_{33=}=-486 & \mathrm{~d}_{54}=474 & \mathrm{e}_{31}=3 \\ \mathrm{~d}_{13}=-45 & \mathrm{~d}_{34}=-486 & \mathrm{e}_{11}=7 & \mathrm{e}_{32}=-18 \\ \mathrm{~d}_{14}=285 & \mathrm{~d}_{41}=27 & \mathrm{e}_{12}=2 & \mathrm{e}_{33}=45 \\ \mathrm{~d}_{21}=38 \mathrm{~L} & \mathrm{e}_{13}=1 & \mathrm{e}_{34}=0 \\ \mathrm{~d}_{22}=18 \mathrm{~L} & \mathrm{~d}_{42}=-243 & \mathrm{e}_{14}=0 & \mathrm{e}_{41}=7 \\ \mathrm{~d}_{23}=18 \mathrm{~L} & \mathrm{~d}_{43}=2673 & \mathrm{e}_{21}=3 & \mathrm{e}_{42}=-20 \\ \mathrm{~d}_{24}=38 \mathrm{~L} & \mathrm{~d}_{44}=567 & \mathrm{e}_{22}=36 & \mathrm{e}_{43}=23 \\ \mathrm{~d}_{31}=54 & \mathrm{~d}_{51}=38 & \mathrm{e}_{23}=-9 & \mathrm{e}_{44}=80 / \mathrm{L}\end{array}$


Novamente lembrando que os termos "d" devem ser multiplicados por $\frac{\mathrm{L}}{6720}$ e os termos em"e" por $\frac{\mathrm{L}}{80}$.

Nota-se que em determinados trechos das matrizes [K] e [Q], aparecem elementos dispostos em diagonal que possuem o mesmo valor absoluto, porém apresentam sinais inversos. Esses elementos são coeficientes correspondentes as coordenadas de rotação em torno dos eixos. Como no modelo apresentado, as coordenadas de rotação em torno dos eixos $X_{1}$ e $X_{2}$ apresentam sinais opostos, seus coeficientes também apresentaram sinais contrários.

E os vetores são dados da seguinte forma:

$$
\begin{aligned}
& \{\mathrm{F}\}^{\mathrm{T}}=\left\{\begin{array}{lllllllllllllll}
\mathrm{F}_{1} & \mathrm{~F}_{2} & \mathrm{~F}_{3} & \mathrm{M}_{2} & \mathrm{M}_{1} & 0 & 0 & 0 & 0 & 0 & 0 & 0 & 0 & 0 & 0
\end{array}\right\} \\
& \left\{\mathrm{u}_{\mathrm{p}}\right\}^{\mathrm{T}}=\left\{\begin{array}{llllllllllllll}
\mathrm{u}_{\mathrm{i}} & \mathrm{v}_{\mathrm{i}} & \mathrm{w}_{\mathrm{i}} & \mathrm{u}_{\mathrm{i}} \mathrm{v}^{\prime}{ }_{\mathrm{i}} & \mathrm{u}_{\mathrm{j}} & \mathrm{v}_{\mathrm{j}} & \mathrm{w}_{\mathrm{j}} & \mathrm{u}_{\mathrm{k}} & \mathrm{v}_{\mathrm{k}} & \mathrm{w}_{\mathrm{k}} & \mathrm{u}_{1} & \mathrm{v}_{1} & \mathrm{w}_{1}
\end{array}\right\} \\
& \left.\left\{\mathrm{P}_{\mathrm{p}}\right\}^{\mathrm{T}}=\begin{array}{llllllllllllll}
\mathrm{P}_{1} & \mathrm{P}_{5} & \mathrm{P}_{9} & \mathrm{P}_{2} & \mathrm{P}_{6} & \mathrm{P}_{10} & \mathrm{P}_{3} & \mathrm{P}_{7} & \mathrm{P}_{11} & \mathrm{P}_{4} & \mathrm{P}_{8} & \mathrm{P}_{12}
\end{array}\right\}
\end{aligned}
$$

Das equações provenientes do MEC, sabemos que

$$
\mathrm{C}_{\mathrm{ij}} \mathrm{u}_{\mathrm{i}}=-\int_{\Gamma} \mathrm{p}_{\mathrm{ij}}^{*} \mathrm{u}_{\mathrm{j}} \mathrm{d} \Gamma+\int_{\Gamma} \mathrm{u}_{\mathrm{ij}}^{*} \mathrm{p}_{\mathrm{j}} \mathrm{d} \Gamma+\int_{\Omega} \mathrm{u}_{\mathrm{ij}}^{*} \mathrm{~b}_{\mathrm{j}} \mathrm{d} \Omega
$$

Entretanto, para o caso de estacas imersas em um meio contínuo, é necessário adicionar um termo na equação geral de deslocamentos, correspondente a ação de uma carga distribuída ao longo das estacas, sendo feito o acréscimo a partir do limite de forças volumétricas. Desta maneira, a nova equação de deslocamentos fica:

$$
\mathrm{C}_{\mathrm{ij}} \mathrm{u}_{\mathrm{i}}=-\int_{\Gamma} \mathrm{p}_{\mathrm{ij}}^{*} \mathrm{u}_{\mathrm{j}} \mathrm{d} \Gamma+\int_{\Gamma} \mathrm{u}_{\mathrm{ij}}^{*} \mathrm{p}_{\mathrm{j}} \mathrm{d} \Gamma+\int_{\Omega} \mathrm{u}_{\mathrm{ij}}^{*} \mathrm{~b}_{\mathrm{j}} \mathrm{d} \Omega+\int_{\Gamma_{\varepsilon}} \mathrm{u}_{\mathrm{ij}}^{*} \mathrm{q}_{\mathrm{j}}^{\mathrm{e}} \mathrm{d} \Gamma
$$

onde

$\mathrm{q}_{\mathrm{j}}^{\mathrm{e}}$ : Forças de interação aplicadas ao sólido tridimesional

$\Gamma_{\mathrm{e}}$ : Linhas de carga onde estão aplicadas as forças $\mathrm{q}_{\mathrm{j}}^{\mathrm{e}}$ 
$\mathrm{Na}$ equação (3.30), a parcela referente às forças volumétricas pode ser desprezada para os efeitos desse estudo, e como será empregada a solução de Mindlin para deslocamentos,e não serão analisados casos de escavações, a $2^{\mathrm{a}}$ e a $3^{\mathrm{a}}$ parcela também serão desconsideradas. Assim, temos:

$$
\mathrm{C}_{\mathrm{ij}} \mathrm{u}_{\mathrm{i}}=\int_{\Gamma_{\varepsilon}} \mathrm{u}_{\mathrm{ij}}^{*} \mathrm{q}_{\mathrm{j}}^{\mathrm{e}} \mathrm{d} \Gamma
$$

Lembrando que para as equações de Mindlin, $\mathrm{C}_{\mathrm{ij}}=[\mathrm{I}]$,

$$
\mathrm{u}_{\mathrm{i}}=\int_{\Gamma_{\varepsilon}} \mathrm{u}_{\mathrm{ij}}^{*} \mathrm{q}_{\mathrm{j}}^{\mathrm{e}} \mathrm{d} \Gamma
$$

Agora se faz necessário resolver a integral da equação (3.32). Como a estaca, nesse estudo será tratado como um elemento de barra, imerso num meio semiinfinito, isótropo, homogêneo e elástico linear, e que a menor distância entre o ponto fonte e o ponto campo será igual ao raio da estaca, não havendo, portanto qualquer singularidade na integração da mesma, utilizaremos o artifício da integração pela regra da quadratura de Gauss. Para evitar a utilização de integração com muitos pontos de Gauss, porém, sem perder o numero de contribuições dos valores dos coeficientes de influencia do solo ao longo da estaca, utilizaremos também o artifício da subelementação.

Assim, equação (2.32) pode ser escrita como:

$$
u_{i}=\int_{\Gamma e} u_{i j}^{*}(s, p) \bar{\phi}_{j}(p) d \Gamma(p) P_{j}(p)
$$

Pela regra da quadratura de Gauss:

$$
\mathrm{u}_{\mathrm{i}}=\frac{\mathrm{L}}{2} \sum_{\mathrm{k}=1}^{\mathrm{ng}} \mathrm{u}_{\mathrm{ij}}^{*}(\mathrm{~s}, \mathrm{p}) \bar{\phi}_{\mathrm{j}}(\mathrm{p}) \mathrm{w}_{\mathrm{k}} \mathrm{P}_{\mathrm{j}}(\mathrm{p})
$$

onde

ng: É o número de pontos de Gauss;

$\mathrm{W}_{\mathrm{k}}$ : É peso de ponderação da integração do k-ésimo ponto. 


$$
\left\{\mathrm{u}_{\mathrm{s}}\right\}=\sum\left[\int\left[\mathrm{U}^{*}\right]\{\hat{\phi}\} \mathrm{d} \Gamma\right]\left\{\mathrm{P}_{\mathrm{s}}\right\}
$$

onde

$\left\{u_{s}\right\}$ : é o vetor de deslocamentos dos pontos de colocação;

$\left[\mathrm{U}^{*}\right]$ : é matriz de soluções fundamentais de Mindlin;

$\{\bar{\phi}\}$ : é o vetor de funções de forma para as forças de interação;

$\left\{\mathrm{P}_{\mathrm{s}}\right\}$ : é o vetor de forças na interface estaca-solo;

ou ainda escrevendo na forma matricial:

$$
\begin{aligned}
& \left\{\mathrm{u}_{\mathrm{s}}\right\}_{12 \times 1}=[\mathrm{G}]_{12 \times 12}\left\{\mathrm{P}_{\mathrm{s}}\right\}_{12 \times 1} \\
& {\left[\begin{array}{c}
\mathrm{u}_{\mathrm{i}} \\
\mathrm{v}_{\mathrm{j}} \\
\mathrm{w}_{\mathrm{k}} \\
\vdots
\end{array}\right]_{\mathrm{S}}\left[\begin{array}{cccc}
\int_{\Gamma_{\mathrm{e}}} \mathrm{u}_{11} \bar{\phi}_{1} \mathrm{~d} \Gamma & \int_{\Gamma_{\mathrm{e}}} \mathrm{u}_{12} \bar{\phi}_{2} \mathrm{~d} \Gamma & \int_{\Gamma_{\mathrm{e}}} \mathrm{u}_{13} \bar{\varphi}_{3} \mathrm{~d} \Gamma & \ldots \\
\int_{\Gamma_{\mathrm{e}}}^{\mathrm{u}_{21}} \bar{\phi}_{1} \mathrm{~d} \Gamma & \int_{\Gamma_{\mathrm{e}}}^{\mathrm{u}} \mathrm{u}_{22} \bar{\phi}_{2} \mathrm{~d} \Gamma & \int_{\Gamma_{\mathrm{e}}}^{\mathrm{u}} \mathrm{u}_{23} \varphi_{3} \mathrm{~d} \Gamma & \ldots \\
\int_{\Gamma_{\mathrm{e}}}^{\mathrm{u}_{31}} \bar{\phi}_{1} \mathrm{~d} \Gamma & \int_{\Gamma_{\mathrm{e}}}^{\mathrm{u}} \mathrm{u}_{32} \bar{\phi}_{2} \mathrm{~d} \Gamma & \int_{\Gamma_{\mathrm{e}}}^{\mathrm{u}_{33} \varphi_{3} \mathrm{~d} \Gamma} & \ldots \\
\vdots & \vdots & \vdots & \ddots
\end{array}\right] \cdot\left[\begin{array}{c}
\mathrm{P}_{1} \\
\mathrm{P}_{5} \\
\tau_{1} \\
\vdots
\end{array}\right]_{\mathrm{S}}}
\end{aligned}
$$

onde:

$$
\lceil\mathrm{G}\rceil=\sum \int_{\Gamma_{\varepsilon}}\left[\mathrm{U}^{*}\right]\{\bar{\phi}\} \mathrm{d} \Gamma
$$

Conforme foi visto anteriormente, a contribuição do solo para o sistema é feito através da integração das soluções fundamentais de Mindlin. Os termos da matriz [G] são calculados através da multiplicação da integral da solução fundamental $\mathrm{u}_{\mathrm{ij}}$ pela função correspondente às forças de interface (ao longo do fuste) ou tensão normal (na base da estaca). Contudo um melhor entendimento, é necessário discutir algumas etapas do processo:

- Os coeficientes da matriz [G] são referentes a integração ao longo do fuste da estaca são dados através de uma integração dupla, quando na 
direção $X_{3}$, sendo desenvolvida analiticamente de 0 a $2 \pi$ e numericamente ao longo do eixo da estaca, utilizando a regra da quadratura de Gauss. Já nas direções $\mathrm{X}_{1}$ e $\mathrm{X}_{2}$, as integrais são resolvidas numericamente, utilizando a regra da quadratura de Gauss.

Quando o ponto fonte e o ponto campo forem iguais (situação em que ocorre a contribuição da tensão normal na base da estaca, no 4 ponto de colocação), o termo $\mathrm{G}_{12 \times 12}$ será calculado através de uma integral dupla numérica, utilizando a regra da quadratura de Gauss, ou seja, será integrada numericamente no intervalo de 0 a $2 \pi$ e novamente utilizando outra integral numérica no intervalo de 0 à $\mathrm{r}$.

Retomando agora a equação (3.25):

$$
[\mathrm{K}]\left\{\mathrm{u}_{\mathrm{p}}\right\}=\{\mathrm{F}\}-[\mathrm{Q}]\left\{\mathrm{P}_{\mathrm{p}}\right\}
$$

Das equações (3.25) e (3.34) temos um conjunto de expressões algébricas que possuem alguns termos em comum. Abaixo descreve-se cada vetor e matriz, que compõem estas expressões, bem como algumas relações entre os mesmo:

$[\mathrm{K}]$ : Matriz de rigidez da estaca;

$\left\{\mathrm{u}_{\mathrm{p}}\right\}$ : Vetor de deslocamentos laterais da estaca;

$\{\mathrm{F}\}$ : Vetor de forças na cabeça da estaca;

[Q]: Matriz de transformação de cargas do elemento em cargas nodais;

$\left\{\mathrm{P}_{\mathrm{p}}\right\}$ : Vetor de forças de interação composto por todos os nos da estaca;

$\left\{\mathrm{u}_{\mathrm{s}}\right\}$ : Vetor de deslocamentos laterais do solo;

[G]: Matriz de soluções fundamentais do solo;

$\left\{\mathrm{P}_{\mathrm{s}}\right\}$ : Vetor de forças de interação na interface estaca-solo.

Isolando o termo $\left\{\mathrm{P}_{\mathrm{s}}\right\}$ na equação (3.34)

$$
\left\{\mathrm{P}_{\mathrm{s}}\right\}_{12 \times 1}=[\mathrm{G}]_{12 \times 12^{-1}}\left\{\mathrm{u}_{\mathrm{s}}\right\}_{12 \times 1}
$$

Realizando o equilíbrio na interface solo-estaca, temos:

$$
\left\{\mathrm{u}_{\mathrm{s}}\right\}=\left\{\mathrm{u}_{\mathrm{p}}\right\}
$$




$$
\left\{\mathrm{P}_{\mathrm{s}}\right\}+\left\{\mathrm{P}_{\mathrm{p}}\right\}=0
$$

e substituindo (3.36) em (3.25), temos:

$$
[\mathrm{K}]_{14 \times 14}\left\{\mathrm{u}_{\mathrm{p}}\right\}_{14 \times 1}=\{\mathrm{F}\}_{14 \times 1}-[\mathrm{Q}]_{14 \times 12}[\mathrm{G}]_{12 \times 12}^{-1}\left\{\mathrm{u}_{\mathrm{s}}\right\}_{12 \times 1}
$$

Fazendo agora:

$$
[\mathrm{T}]_{14 \times 12}=[\mathrm{Q}]_{14 \times 12}[\mathrm{G}]_{12 \times 12}^{-1}
$$

Para tornar algebricamente possível o equacionamento da expressão (3.39), necessita-se expandir a matriz [T] adicionando duas colunas de zeros nos termos referentes as rotações em torno dos eixos $\mathrm{X}_{1}$ e $\mathrm{X}_{2}$. Assim adicionam-se duas colunas de zeros a partir da terceira coluna da matriz [T]: Conseqüentemente, o vetor $\left\{u_{s}\right\}$ também deve ser expandido:

$$
\therefore[\overline{\mathrm{T}}]_{14 \times 14}=\left[\begin{array}{cccccccc}
\mathrm{T}_{11} & \mathrm{~T}_{12} & \mathrm{~T}_{13} & 0 & 0 & \ldots & \mathrm{T}_{113} & \mathrm{~T}_{114} \\
\mathrm{~T}_{21} & \mathrm{~T}_{22} & \mathrm{~T}_{23} & 0 & 0 & \ldots & \mathrm{T}_{213} & \mathrm{~T}_{214} \\
\ldots & \ldots & \ldots & 0 & 0 & \ldots & \ldots & \ldots \\
\ldots & \ldots & \ldots & 0 & 0 & \ldots & \ldots & \ldots \\
\ldots & \ldots & \ldots & 0 & 0 & \ldots & \ldots & \ldots \\
\ldots & \ldots & \ldots & 0 & 0 & \ldots & \ldots & \ldots \\
\mathrm{T}_{131} & \mathrm{~T}_{132} & \mathrm{~T}_{133} & 0 & 0 & \ldots & \mathrm{T}_{1313} & \mathrm{~T}_{1314} \\
\mathrm{~T}_{141} & \mathrm{~T}_{142} & \mathrm{~T}_{143} & 0 & 0 & \ldots & \mathrm{T}_{1413} & \mathrm{~T}_{1414}
\end{array}\right]
$$

Assim, o sistema final fica:

$$
\begin{aligned}
& {[\mathrm{K}]_{14 \times 14}\{\mathrm{U}\}_{14 \times 1}=\{\mathrm{F}\}_{14 \times 1}-[\overline{\mathrm{T}}]_{14 \times 14}\{\mathrm{U}\}_{14 \times 1}} \\
& {[[\mathrm{~K}]+[\overline{\mathrm{T}}]]\{\mathrm{U}\}=\{\mathrm{F}\}} \\
& {[\overline{\mathrm{K}}]\{\mathrm{U}\}=\{\mathrm{F}\}}
\end{aligned}
$$




\subsubsection{ESTACAS INCLINADAS}

Toda formulação descrita anteriormente, bem como as matrizes e vetores obtidos foram baseados em um sistema dextrorso que tem como um dos eixos principais, o próprio eixo da estaca. Porém, nos problemas de engenharia, nem sempre as estacas de fundação são elementos verticais, podendo apresentar certo grau de inclinação em relação ao eixo vertical ou mesmo diferentes inclinações entre as várias estacas de um mesmo elemento de fundação. Para podermos tratar do assunto estaca inclinada, é necessário adotar um novo sistema de referência, idêntico para todas elas, uma vez que as mesmas podem ter diferentes inclinações entre si. Assim, nesta etapa, desenvolve-se uma nova formulação, tomando como partida as relações obtidas no item anterior.

Todas as formulações desenvolvidas nos itens anteriores, que estão relacionadas ao sistema dextrorso, na qual um dos eixos principais foi tomado como o próprio eixo da estaca, será a partir de agora admitida como sendo relacionada ao eixo local de referência. $\mathrm{O}$ sistema global de referência será tomado em relação aos eixos $\mathrm{X}_{1}, \mathrm{X}_{2}$, e $\mathrm{X}_{3}$, e um índice " $\mathrm{g}$ " será adotado para todas as matrizes e vetores cujas direções estiverem relacionadas aos referidos eixos.

Por comodidade, optou-se por trabalhar com a montagem das matrizes [K], [Q] e [G] referidas aos eixos locais, fazendo-se as devidas alterações na formulação das mesmas, quando necessário. A passagem para o sistema de referência global somente é realizada na etapa anterior à resolução do sistema linear final.

Da equação (3.33d) dos deslocamentos do solo, sabe-se que a matriz [G] relaciona as forças de interação do solo aos deslocamentos do mesmo. Porém, a formulação admite que os deslocamentos e as forças de interação são aproximados por uma certa função polinomial que varia na extensão do fuste, ou seja, os vetores $\left\{\mathrm{u}_{\mathrm{s}}\right\}$ e $\left\{\mathrm{P}_{\mathrm{s}}\right\}$ estão associados ao sistema local de referência.

Porém sabe-se que as equações fundamentais de Mindlin fazem referência ao global, isto é, as equações da matriz [G] foram descritas no sistema global, sendo assim necessário rotacionar esse sistema para adequá-la ao problema. 
FERRO apresentou em seu trabalho, uma matriz de rotação para sistemas tridimensionais.

$$
[\mathrm{R}]=\left[\begin{array}{ccc}
\sqrt{\mathrm{CY}^{2}+\mathrm{CZ}^{2}} & \frac{-\mathrm{CXCY}}{\sqrt{\mathrm{CY}^{2}+\mathrm{CZ}^{2}}} & \frac{-\mathrm{CXCZ}}{\sqrt{\mathrm{CY}^{2}+\mathrm{CZ}^{2}}} \\
0 & \frac{\mathrm{CZ}}{\sqrt{\mathrm{CY}^{2}+\mathrm{CZ}^{2}}} & \frac{-\mathrm{CY}}{\sqrt{\mathrm{CY}^{2}+\mathrm{CZ}^{2}}} \\
\mathrm{CX} & \mathrm{CY} & \mathrm{CZ}
\end{array}\right]
$$

Sendo:

$$
\mathrm{CX}=\frac{\mathrm{x}_{1}-\mathrm{x}_{\mathrm{i}}}{\mathrm{L}} ; \mathrm{Cy}=\frac{\mathrm{y}_{1}-\mathrm{y}_{\mathrm{i}}}{\mathrm{L}} ; \mathrm{Cz}=\frac{\mathrm{z}_{1}-\mathrm{z}_{\mathrm{i}}}{\mathrm{L}} ;
$$

Onde os índices " $\mathrm{l}$ " e " $\mathrm{i}$ " das coordenadas $\mathrm{x}, \mathrm{y}, \mathrm{e} \mathrm{z}$ fazem referencia ao ultimo e ao primeiro ponto de colocação de uma mesma estaca e L representa o comprimento da mesma.

Assim pode-se relacionar os vetores de deslocamentos da seguinte forma:

$$
\begin{aligned}
& \left\{\mathrm{u}_{\mathrm{s}}\right\}=[\mathrm{R}]\left\{\mathrm{u}_{\mathrm{s}}\right\}_{\mathrm{g}} \text { e }\left\{\mathrm{P}_{\mathrm{s}}\right\}=[\mathrm{R}]\left\{\mathrm{P}_{\mathrm{s}}\right\}_{\mathrm{g}} \\
& \text { ou } \\
& \left\{\mathrm{u}_{\mathrm{s}}\right\}_{\mathrm{g}}=[\mathrm{R}]^{-1}\left\{\mathrm{u}_{\mathrm{s}}\right\} \text { e }\left\{\mathrm{P}_{\mathrm{s}}\right\}_{\mathrm{g}}=[\mathrm{R}]^{-1}\left\{\mathrm{P}_{\mathrm{s}}\right\}
\end{aligned}
$$

Nota-se que para estacas com grau de inclinação zero (estacas verticais), $\mathrm{Cx}=\mathrm{Cy}=0, \mathrm{Cz}=1$ e $[\mathrm{R}]=[\mathrm{I}]$, ou seja, os sistemas de referencia global e local se sobrepõem.

Retornando ao problema original, faz-se necessário rotacionar o tensor de deslocamentos para adequar às coordenadas locais, para posterior integração das soluções fundamentais escritas no novo sistema de referência.

Da equação de deslocamentos do solo, sabe-se que:

$$
\begin{aligned}
& \left\{u_{s}\right\}_{g}=[G]_{g}\left\{P_{s}\right\}_{g} \\
& \left(\begin{array}{c}
u \\
v \\
w
\end{array}\right)_{g}=\int_{\Gamma_{e}}\left(\begin{array}{lll}
u_{11} & u_{12} & u_{13} \\
u_{21} & u_{22} & u_{23} \\
u_{31} & u_{32} & u_{33}
\end{array}\right) \cdot\left(\begin{array}{ccc}
\phi X_{1} & 0 & 0 \\
0 & \phi_{X 2} & 0 \\
0 & 0 & \phi_{X 3}
\end{array}\right) d \Gamma_{e} \cdot\left(\begin{array}{c}
P_{1} \\
P_{2} \\
P_{3}
\end{array}\right)_{g}
\end{aligned}
$$




$$
\left\{\mathrm{u}_{\mathrm{s}}\right\}_{\mathrm{g}}=\left[\mathrm{U}^{*}\right][\phi]\left\{\mathrm{P}_{\mathrm{s}}\right\}_{\mathrm{g}}
$$

Substituindo os vetores $\left\{\mathrm{u}_{\mathrm{s}}\right\}_{\mathrm{g}}$ e $\left\{\mathrm{P}_{\mathrm{s}}\right\}_{\mathrm{g}}$ pelas relações dadas em (3.43), temos

$$
[\mathrm{R}]^{-1}\left\{\mathrm{u}_{\mathrm{s}}\right\}=\left[\mathrm{U}^{*}\right][\phi][\mathrm{R}]^{-1}\{\mathrm{Ps}\}
$$

Lembrando que $[R]$ é uma matriz ortogonal, ou seja: $[R]^{-1}=[R]^{T}$ :

$$
\begin{aligned}
& \left\{\mathrm{u}_{\mathrm{s}}\right\}=[\mathrm{R}]\left[\mathrm{U}^{*}\right][\mathrm{R}]^{\mathrm{T}}[\phi]\{\mathrm{Ps}\} \\
& \left\{\mathrm{u}_{\mathrm{s}}\right\}=[\mathrm{G}]\{\mathrm{Ps}\}
\end{aligned}
$$

E assim, obtêm-se a nova matriz [G], relacionada aos eixos locais, onde:

$$
[\mathrm{G}]=[\mathrm{R}]\left[\mathrm{U}^{*}\right][\mathrm{R}]^{\mathrm{T}}[\phi]
$$

A seguir, trabalhando no sistema local, repete-se os todos os procedimentos descritos pelas equações (3.36) até (3.41c). Para obter o sistema final nas coordenadas globais, basta fazer um rearranjo:

$$
[\beta]^{\mathrm{T}}[\overline{\mathrm{K}}][\beta]\{\mathrm{U}\}_{\mathrm{g}}=\{\mathrm{F}\}_{\mathrm{g}}
$$

onde

$$
[\beta]_{14 \times 14}=\left[\begin{array}{cccccc}
{[\mathrm{R}]_{3 \times 3}} & 0 & 0 & {[0]_{3 \times 3}} & {[0]_{3 \times 3}} & {[0]_{3 \times 3}} \\
0 & 1 & 0 & 0 & 0 & 0 \\
0 & 0 & 1 & 0 & 0 & 0 \\
{[0]_{3 \times 3}} & 0 & 0 & {[\mathrm{R}]_{3 \times 3}} & {[0]_{3 \times 3}} & {[0]_{3 \times 3}} \\
{[0]_{3 \times 3}} & 0 & 0 & {[0]_{3 \times 3}} & {[\mathrm{R}]_{3 \times 3}} & {[0]_{3 \times 3}} \\
{[0]_{3 \times 3}} & 0 & 0 & {[0]_{3 \times 3}} & {[0]_{3 \times 3}} & {[\mathrm{R}]_{3 \times 3}}
\end{array}\right]
$$

\subsection{GRUPO DE ESTACAS}

Nos itens anteriores, desenvolveu-se toda a formulação para estacas isoladas. Nesta seção, descrevem-se as modificações necessárias para a consideração de um sistema composto por um grupo de estacas. 
O sistema de equações para um grupo de estacas é bastante semelhante ao sistema de uma estaca isolada. A grande diferença está na ordem das matrizes envolvidas. De uma maneira simplificada pode-se descrever todo processo partindo do sistema final de uma estaca isolada, mudando apenas a ordem das matrizes envolvidas.

Assim com nos trabalhos de POULOS (1968, 1971b), em um sistema composto por várias estacas, será considerada a superposição elástica da influência de todos os elementos do grupo, sempre tomados dois a dois, ou seja, o sistema final será composto por vários subsistemas que por sua vez, representarão a interação de duas estacas e o solo, ou a influência de uma estaca sobre ela mesma.

Entretanto, algumas considerações serão feitas a seguir, para este modelo idealizado:

- A influência de uma estaca sobre si mesma será feita através da integração numérica pela regra da quadratura de Gauss, com os pontos posicionados no eixo da estaca e os pontos campo, no contorno da mesma.

- A integração entre estacas diferentes também será feita através da regra da quadratura de Gauss, porém agora, os pontos campo e fonte serão dispostos no eixo das diferentes estacas.

Assim para a interação entre duas estacas, temos

$$
[\mathrm{K}]_{28 \times 28}\left\{\mathrm{u}_{\mathrm{p}}\right\}_{28 \times 1}=\{\mathrm{F}\}_{28 \times 11^{-}}[\mathrm{Q}]_{28 \times 28}[\mathrm{G}]_{24 \times 24}^{-1}\left\{\mathrm{u}_{\mathrm{s}}\right\}_{24 \times 1}
$$

Onde:

$$
[\mathrm{G}]_{24 \times 24}=\left[\begin{array}{ll}
{\left[\mathrm{MEC}_{11}\right]_{12 \times 12}} & {\left[\mathrm{MEC}_{12}\right]_{12 \times 12}} \\
{\left[\mathrm{MEC}_{21}\right]_{12 \times 12}} & {\left[\mathrm{MEC}_{22}\right]_{12 \times 12}}
\end{array}\right]
$$

$\mathrm{MEC}_{11}$ : Representa os coeficientes de rigidez provenientes do solo relativos 
a influencia da estaca 1 sobre ela mesma

MEC $_{12}$ Representa os coeficientes de rigidez provenientes do solo relativos a influencia da estaca 1 sobre a estaca 2

$\mathrm{MEC}_{21}$ Representa os coeficientes de rigidez provenientes do solo relativos a influencia da estaca 2 sobre a estaca 1

$\mathrm{MEC}_{22}$ Representa os coeficientes de rigidez provenientes do solo relativos a influencia da estaca 2 sobre ela mesma

$[\mathrm{K}]_{28 \times 28}=\left[\begin{array}{cc}{\left[\mathrm{MEF}_{11}\right]_{14 \times 14}} & {[0]_{14 \times 14}} \\ {[0]_{14 \times 14}} & {\left[\mathrm{MEF}_{22}\right]_{14 \times 14}}\end{array}\right]$

$\mathrm{MEF}_{11}$ : Representa os coeficientes de rigidez provenientes da estaca relativos a influencia da estaca 1 sobre ela mesma

$\mathrm{MEF}_{22}$ Representa os coeficientes de rigidez provenientes da estaca relativos a influencia da estaca 2 sobre ela mesma

$[\mathrm{Q}]_{28 \times 24}=\left[\begin{array}{cc}{\left[\mathrm{Q}_{11}\right]_{14 \times 12}} & {[0]_{14 \times 12}} \\ {[0]_{14 \times 12}} & {\left[\mathrm{Q}_{22}\right]_{14 \times 12}}\end{array}\right]$

$\mathrm{Q}_{11}$ : Coeficientes das forças de interação na estaca 1

$\mathrm{Q}_{22}$ Coeficientes das forças de interação na estaca 2

Para um grupo com mais de duas estacas, o procedimento padrão é o mesmo, bastando apenas montar as matrizes considerando cada estacas isolada, e as matrizes das interações entre uma estaca e as demais, sempre tomadas dois a dois, e inserindoas na matriz do sistema final. 


\section{SISTEMA CASCA-SOLO}

\subsection{INTRODUÇÃO}

Um sistema estrutural pode ser dividido em subestruturas colaborantes, que por sua vez podem ser compostos por elementos estruturais, ou até mesmo serem os próprios. Desta forma, os elementos estruturais podem ser classificados da seguinte forma, quanto às suas dimensões:

- Elementos lineares: quando uma das suas dimensões é muito superior que as demais.

- Elementos laminares: quando uma de suas dimensões é menor, se comparada com as outras duas.

- Elementos volumétricos: quando todas as suas dimensões possuem a mesma ordem de grandeza.

Na engenharia é muito comum a utilização de elementos estruturais de casca. Uma casca é uma estrutura caracterizada pela superfície média, definida como a região formada pelos pontos eqüidistantes das superfícies limitantes. Dessa forma, uma casca é facilmente definida através do conhecimento da sua espessura e da sua superfície média. 
Por apresentar a espessura muito menor que as outras dimensões, o elemento de casca pode ser aproximado por uma ou mais lâminas com superfícies planas, simplificando o problema, obtendo-se um modelo bidimensional menos complexo.

De maneira geral, o elemento de casca pode ser simulado através da sobreposição dos efeitos de um estado de flexão e de um estado de membrana. $\mathrm{O}$ estado de membrana é caracterizado por uma distribuição uniforme de tensões ao longo da espessura resultando em esforços normais e tangenciais. Já o estado de flexão é conseqüência de uma distribuição de tensões não uniforme ao longo da espessura, resultando em esforços que tendem a fletir e a cisalhar a estrutura.

Neste trabalho a casca será discretizada em elementos planos triangulares.O efeito de flexão será computada através da adoção do elemento finito DKT (Discrete Kirchhoff Theory) - BATOZ \& BATHE (1980) e a membrana através do elemento FF (Formulação Livre) - BERGAN \& FELIPPA (1985). Não será desenvolvida a formulação dos elementos, uma vez que ambos são de conhecimento amplamente difundido nos meios acadêmicos e científicos.

\subsection{O ELEMENTO DKTFF}

A formulação proposta para o elemento laminar desse trabalho, é desenvolvida admitindo-se a hipóteses de Kirchhoff-Love:

- Os deslocamentos são muito pequenos se comparados em relação a espessura da lâmina.

- As tensões normais à superfície média são desprezíveis em relação as demais.

- A espessura da lâmina é pequena em relação às dimensões e aos raios de curvatura da superfície média. 
- Os pontos pertencentes a uma mesma reta normal à superfície média na situação indeformada, encontram-se em uma mesma reta normal à superfície deformada.

Com base nas hipóteses acima, os deslocamentos de uma área infinitesimal de um elemento laminar são dados por:

$$
\{u\}=\left(\begin{array}{c}
u\left(x_{1}, x_{2}, x_{3}\right) \\
v\left(x_{1}, x_{2}, x_{3}\right) \\
w\left(x_{1}, x_{2}, x_{3}\right)
\end{array}\right)=\left[\begin{array}{c}
\mathrm{u}_{0} \cdot\left(\mathrm{x}_{1}, \mathrm{x}_{2}\right)-\mathrm{x}_{3} \cdot \frac{\mathrm{d}}{\mathrm{dx}_{1}} \mathrm{w}_{0} \\
\mathrm{v} \cdot\left(\mathrm{x}_{1}, \mathrm{x}_{2}\right)-\mathrm{x}_{3} \cdot \frac{\mathrm{d}}{\mathrm{dx}_{2}} \mathrm{w}_{0} \\
\mathrm{w} \cdot\left(\mathrm{x}_{1}, \mathrm{x}_{2}\right)
\end{array}\right]
$$

onde $\mathrm{u}_{0}, \mathrm{v}_{0}$ e $\mathrm{w}_{0}$ são os campos de deslocamentos locais de um ponto genérico, considerado nas direções $\mathrm{x}_{1}, \mathrm{x}_{2}$ e $\mathrm{x}_{3}$.

As parcelas do campo das deformações oriunda dos efeitos de membrana e flexão são dadas logo abaixo:

$$
\{\varepsilon\}=\{\varepsilon\}_{\mathrm{f}}+\{\varepsilon\}_{\mathrm{m}}=\left(\begin{array}{c}
\varepsilon_{1} \\
\varepsilon_{2} \\
\gamma_{12}
\end{array}\right)=\left(\begin{array}{c}
\frac{\mathrm{d}}{\mathrm{dx}_{1}} \mathrm{u}_{0} \\
\frac{\mathrm{d}}{\mathrm{dx}_{2}} \mathrm{v}_{0} \\
\frac{\mathrm{d}}{\mathrm{dx}_{2}} \mathrm{u}_{0}+\frac{\mathrm{d}}{\mathrm{dx}} \mathrm{uv} 0
\end{array}\right)-\mathrm{x}_{3}\left(\begin{array}{c}
\frac{\mathrm{d}^{2}}{\mathrm{dx}_{1}{ }^{2}} \mathrm{w}_{0} \\
\frac{\mathrm{d}^{2}}{\mathrm{dx}_{2}{ }^{2}} \mathrm{w}_{0} \\
2 \cdot \frac{\mathrm{d}^{2} \mathrm{w}_{0}}{\mathrm{dx}_{1} \mathrm{dx}_{2}}
\end{array}\right)
$$

onde o índice "m”e "f” fazem referencia à membrana e flexão respectivamente.

Lembrando da relação constitutiva

$$
\{\sigma\}=[\mathrm{D}]\{\varepsilon\}
$$

e aplicando(4.2) sobre a equação (4.3):

$$
\{\sigma\}=[\mathrm{D}]\left(\{\varepsilon\}_{\mathrm{m}}+\{\varepsilon\}_{\mathrm{f}}\right)=\{\sigma\}_{\mathrm{m}}+\{\sigma\}_{\mathrm{f}}
$$


onde [D] é a matriz constitutiva

Torna-se necessario agora, definir um campo de deslocamentos aproximado, para se obter as expressões básicas do método dos elementos finitos.

$$
\left\{\mathrm{u}_{\text {nodal }}\right\}=[\phi] \cdot\{\delta\}=\left(\begin{array}{cc}
\left|\phi_{\mathrm{m}}\right| & |0| \\
|0| & \left|\phi_{\mathrm{f}}\right|
\end{array}\right) \cdot\left(\begin{array}{c}
\left|\delta_{\mathrm{m}}\right| \\
\left|\delta_{\mathrm{f}}\right|
\end{array}\right)
$$

onde $\left[\delta_{\mathrm{m}}\right]$ e $\left[\delta_{\mathrm{f}}\right]$ são os vetores de deslocamentos nodais do elemento, relacionados ao sistema local de coordenadas; $\left[\phi_{\mathrm{m}}\right]$ e $\left[\phi_{\mathrm{f}}\right]$ são as funções de forma para as parcelas de membrana e flexão respectivamente. Para um ponto " $i$ " do elemento, esses vetores podem ser expandidos como:

$$
\begin{aligned}
& {\left[\delta_{\mathrm{m}}\right]^{\mathrm{T}}=\left[\begin{array}{lll}
\mathrm{u}_{\mathrm{i}} & \mathrm{v}_{\mathrm{i}} & \theta_{\mathrm{i}}
\end{array}\right]} \\
& {\left[\delta_{\mathrm{f}}\right]^{\mathrm{T}}=\left[\begin{array}{lll}
\mathrm{w}_{\mathrm{i}} & \theta \mathrm{x}_{1 \mathrm{i}} & \theta \mathrm{x}_{2 \mathrm{i}}
\end{array}\right]}
\end{aligned}
$$

Desta forma, o campo de deformações pode ser expresso como:

$$
\begin{aligned}
& {\left[\delta_{\mathrm{m}}\right]^{\mathrm{T}}=\left[\begin{array}{lll}
\mathrm{u}_{\mathrm{i}} & \mathrm{v}_{\mathrm{i}} & \theta_{\mathrm{i}}
\end{array}\right]} \\
& {\left[\delta_{\mathrm{f}}\right]^{\mathrm{T}}=\left[\begin{array}{lll}
\mathrm{w}_{\mathrm{i}} & \theta \mathrm{x}_{1 \mathrm{i}} & \theta \mathrm{x}_{2 \mathrm{i}}
\end{array}\right]} \\
& \{\varepsilon\}=\left[\left[\mathrm{B}_{\mathrm{m}}\right]+\mathrm{x}_{3} \cdot\left[\mathrm{B}_{\mathrm{f}}\right]\right] \cdot\left\{\mathrm{u}_{\text {nodal }}\right\}=[\mathrm{B}] \cdot\left\{\mathrm{u}_{\text {nodal }}\right\}
\end{aligned}
$$

onde $\left[\mathrm{B}_{\mathrm{m}}\right]$ e $\left[\mathrm{B}_{\mathrm{f}}\right]$ são as matrizes de interpolação de deslocamento-deformação correspondentes à parcela de membrana e flexão.

A expressão da matriz de rigidez pode ser obtida utilizando-se a definição da energia de deformação $\mathrm{U}$ : 


$$
\mathrm{U}=1 / 2 \int_{\mathrm{V}}\{\varepsilon\}^{\mathrm{T}}\{\sigma\} \mathrm{dV}=1 / 2 \int_{\mathrm{V}}\{\varepsilon\}^{\mathrm{T}}[\mathrm{D}]\{\varepsilon\} \mathrm{dV}
$$

Substituindo-se (4.8) em (4.9), tem-se

$$
\mathrm{U}=1 / 2 \int_{\mathrm{V}}\{\delta\}^{\mathrm{T}}[\mathrm{B}]^{\mathrm{T}}[\mathrm{D}][\mathrm{B}]\{\delta\} \mathrm{dV}=1 / 2\{\delta\}^{\mathrm{T}}[\mathrm{K}]\{\delta\} \mathrm{dV}
$$

onde $[\mathrm{K}]$ é a matriz de rigidez definida por:

$$
[K]=\int_{V}[B]^{T}[D][B] d V=\int_{V}\left(\begin{array}{c}
\left|B_{m}\right|^{T} \\
x_{3}\left|B_{f}\right|^{T}
\end{array}\right)\left(\begin{array}{cc}
\left|D_{m}\right| & \left|D_{m f}\right| \\
\left|D_{f m}\right| & \left|D_{f}\right|
\end{array}\right)\left(\left|B_{f}\right|^{T} x_{3}\left|B_{f}\right|^{T}\right)^{2} d V
$$

Resolvendo esta integral, temos:

$$
|K|=\left(\begin{array}{cc}
\left|K_{m}\right| & \left|K_{m f}\right| \\
\left|K_{f m}\right| & \left|K_{f}\right|
\end{array}\right)
$$

onde $\left[\mathrm{K}_{\mathrm{m}}\right]$, $\left[\mathrm{K}_{\mathrm{f}}\right]$, são as matrizes de rigidez de membrana e flexão; $\left[\mathrm{K}_{\mathrm{mf}}\right]$ e $\left[\mathrm{K}_{\mathrm{fm}}\right]$ são matrizes de acomplamento entre as duas ultimas. Suas matrizes são expressas por:

$$
\begin{aligned}
\left|\mathrm{K}_{\mathrm{f}}\right| & =\int_{\mathrm{A}}\left|\mathrm{B}_{\mathrm{f}}\right|^{\mathrm{T}}\left|\mathrm{D}_{\mathrm{f}}\right|\left|\mathrm{B}_{\mathrm{f}}\right| \mathrm{dA} \\
\left|\mathrm{K}_{\mathrm{m}}\right| & =\int_{\mathrm{A}}^{\cdot}\left|\mathrm{B}_{\mathrm{m}}\right|^{\mathrm{T}}\left|\mathrm{D}_{\mathrm{m}}\right|\left|\mathrm{B}_{\mathrm{m}}\right| \mathrm{dA} \\
\left|\mathrm{K}_{\mathrm{mf}}\right| & =\int_{A}^{\cdot}\left|\mathrm{B}_{\mathrm{m}}\right|^{\mathrm{T}}\left|\mathrm{D}_{\mathrm{mf}}\right|\left|\mathrm{B}_{\mathrm{f}}\right| \mathrm{dA} \\
\left|\mathrm{K}_{\mathrm{fm}}\right| & =\int_{\mathrm{A}}\left|\mathrm{B}_{\mathrm{f}}\right|^{\mathrm{T}}\left|\mathrm{D}_{\mathrm{fm}}\right|\left|\mathrm{B}_{\mathrm{m}}\right| \mathrm{dA}
\end{aligned}
$$

sendo: 


$$
\left|D_{m}\right|=\int_{\frac{-h}{2}}^{\frac{h^{\circ}}{2}}|D| d x_{3} \quad\left|D_{f}\right|=\int_{\frac{-h}{2}}^{\frac{h}{2}}\left(x_{3}\right)^{2}|D| d x_{3} \quad\left|D_{m f}\right|=\int_{\frac{-h}{2}}^{\frac{h}{2}} x_{3}|D| d x_{3}
$$

Desta forma, tem-se que $\left[\mathrm{K}_{\mathrm{mf}}\right]=\left[\mathrm{K}_{\mathrm{mf}}\right]=0$, pois

$$
\left|\mathrm{D}_{\mathrm{mf}}\right|=\int_{\frac{-\mathrm{h}}{2}}^{\frac{\mathrm{h}}{2}} \mathrm{x}_{3}|\mathrm{D}| \mathrm{dx}_{3}=0
$$

Assim, a matriz final do elemento de casca é dado por:

$$
|K|=\left(\begin{array}{cc}
\left|K_{m}\right| & |0| \\
|0| & \left|K_{f}\right|
\end{array}\right)
$$

\subsubsection{Efeito de flexão}

Neste trabalho, utiliza-se o elemento DKT para considerar o efeito de flexão nas laminas. O DKT é um elemento finito muito difundido no meio científico e é considerado por muitos, uns dos melhores em sua classe.

O DKT é um elemento triangular com os nós associados em seus vértices. Em cada vértice são associados três graus de liberdade (duas rotações e um deslocamento transversal), totalizando nove deslocabilidades por elemento. Sua formulação inicial adota, além dos nós nos vértices, a existência de nós auxiliares, localizados nos pontos médios dos lados, onde existe apenas um grau de liberdade, representando a rotação da seção transversal, como mostra a figura: 


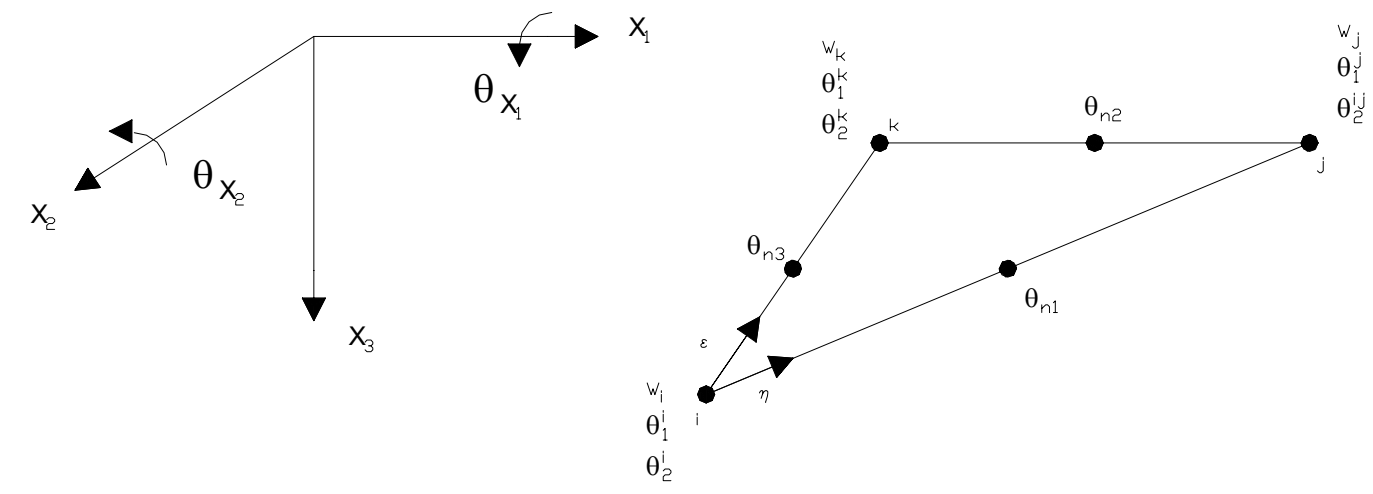

Figura 4.1 - Parâmetros nodais e nós auxiliares do elemento DKT.

A formulação deste elemento finito é inicialmente baseada na teoria de Reissner-Mindlin, considerando o efeito do esforço cortante. Posteriormente, as hipóteses da teoria clássica de placas delgadas, ou teoria de Kirchhoff são impostas discretamente ao longo dos lados do elemento. As hipóteses da teoria de Kirchhoff são:

- Os deslocamentos horizontais dos pontos do plano médio da placa são desprezíveis.

- Todos os pontos contidos em uma reta normal ao plano médio possuem o mesmo deslocamento vertical.

- As tensões normais ao plano médio da placa são desprezíveis.

- Uma reta normal ao plano médio indeformado da placa mantém-se normal à superfície média após a deformação. 
Na teoria de Reissner-Mindlin, porém, os pontos pertencentes a uma reta normal ao plano médio da placa, após a deformação, permanecem sobre a mesma reta, mas não necessariamente normal à superfície deformada.

Por ser um elemento consagrado no meio cientifico, está presente em vários trabalhos publicados no ramo acadêmico. Porém, neste trabalho, não há a pretensão de se desenvolver toda a formulação deste elemento.Todo o desenvolvimento da formulação deste elemento pode ser conferida no trabalho de BATOZ (1980).

\subsubsection{Efeito de membrana}

Para computar o efeito de membrana, utilizou-se neste trabalho, o elemento triangular com formulação livre, desenvolvido por BERGAN \& FELLIPA (1985). Esse elemento de chapa é composto por três nós, localizados nos vértices do mesmo. Em cada nó há três graus de liberdade, sendo duas translações (u e v) nos dois eixos que definem o plano da chapa e uma rotação normal ao plano do elemento $\left(\theta_{3}\right)$.
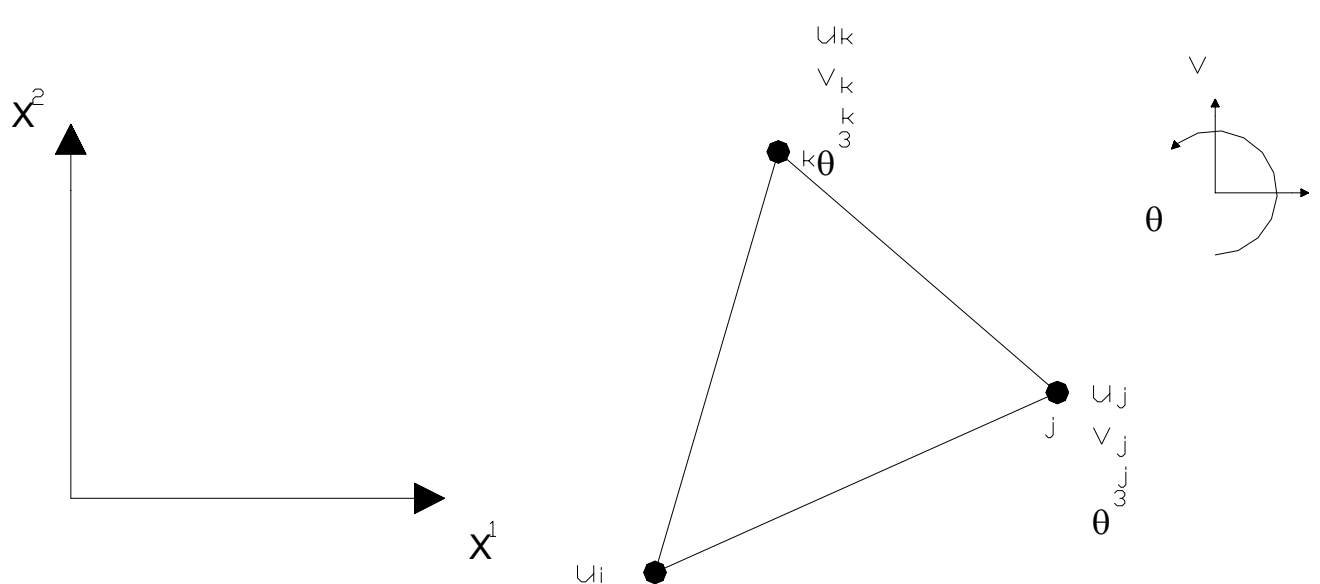

$\theta$

Figura 4.2 - Geometria e graus de liberdade do elemento de membrana. 
Assim como no item anterior, toda a formulação e hipóteses adotadas para o desenvolvimento deste elemento não serão aqui apresentadas. Para maior compreensão, recomenda-se a leitura dos trabalhos de PELETEIRO (1996) e MESQUITA (1998), ambos desenvolvidos no departamento de Engenharia de Estruturas - SET/EESC.

\subsection{O MEIO SEMI-INFINITO}

Nessa etapa é apresentada a contribuição do meio semi-infinito no problema da interação solo-estrutura. Admite-se que o solo está em contato com a estrutura, e que ambas possuem a mesma discretização.

Esse problema incorpora a solução fundamental de Boussinesq-Cerruti, e se as forças de volume forem desprezadas, a representação integral do problema é dada por:

$u_{i}=\int_{\Gamma}^{\cdot} u_{i j}(s, p) \cdot p_{j} d \Gamma(p) \quad(i, j=1,2,3)$

Discretizando-se a superfície do meio semi-infinito em elementos triangulares ou células:

$\mathrm{u}_{\mathrm{i}}=\sum_{\mathrm{n}} \int_{\Omega_{\mathrm{EL}}} \mathrm{u}_{\mathrm{ij}}(\mathrm{s}, \mathrm{p}) \cdot \mathrm{p}_{\mathrm{j}} \mathrm{d} \Omega_{\mathrm{EL}}(\mathrm{p}) \quad(\mathrm{i}, \mathrm{j}=1,2,3)$

onde:

$\Omega_{\mathrm{EL}}$ : domínio do elemento de contorno,

n: número de elementos de células que compõem o contorno $\Gamma$,

$\mathrm{p}_{\mathrm{j}} \quad$ força de superfície na direção “j”. 
$\mathrm{u}_{\mathrm{ij}}$ : $\quad$ soluções fundamentais de Boussinesq-Cerruti, dadas na forma simplificada:

$$
\begin{aligned}
& u_{11}=\frac{1+v}{\pi \cdot E}\left[\frac{1-v}{R}+\frac{v \cdot\left(R_{1}\right)^{2}}{R^{3}}\right] \\
& u_{12}=u_{21}=\frac{v \cdot(1+v) \cdot R_{1} \cdot R_{2}}{\pi \cdot E \cdot R^{3}} \\
& u_{13}=-u_{31}=\frac{(1+v) \cdot(1-2 \cdot v) \cdot R_{1}}{2 \cdot \pi \cdot E \cdot R^{2}} \\
& u_{22}=\frac{1+v}{\pi \cdot E}\left[\frac{1-v}{R}+\frac{v \cdot\left(R_{2}\right)^{2}}{R^{3}}\right] \\
& u_{23}=-u_{32}=\frac{(1+v) \cdot(1-2 \cdot v) \cdot R_{2}}{2 \pi \cdot E \cdot R^{2}} \\
& u_{33}=\frac{1-v}{\pi \cdot E \cdot R}
\end{aligned}
$$

Admitindo-se que as forças de superfície variam linearmente e ao longo do elemento de contorno:

Figura 4.3 - Distribuição das forças de superfície no elemento de contorno 
Assim, a função interpoladora das forças de superfície, pode ser escrita como:

$p_{i}=A_{i} \cdot x_{1 S}+B_{i} \cdot x_{2 S}+C$

onde:

$\mathrm{x}_{1 \mathrm{~S}}, \mathrm{X}_{2 \mathrm{~S}}$ coordenadas do ponto " $\mathrm{s}$ " no sistema global de referência,

$\mathrm{p}_{\mathrm{i}} \quad$ força de superfície na direção “ $\mathrm{i}$ ”.

Fazendo uma translação de eixos, as coordenadas do ponto "s" podem ser escritas em relação à um sistema $\left(\mathrm{x}_{1}, \mathrm{X}_{2}\right)$

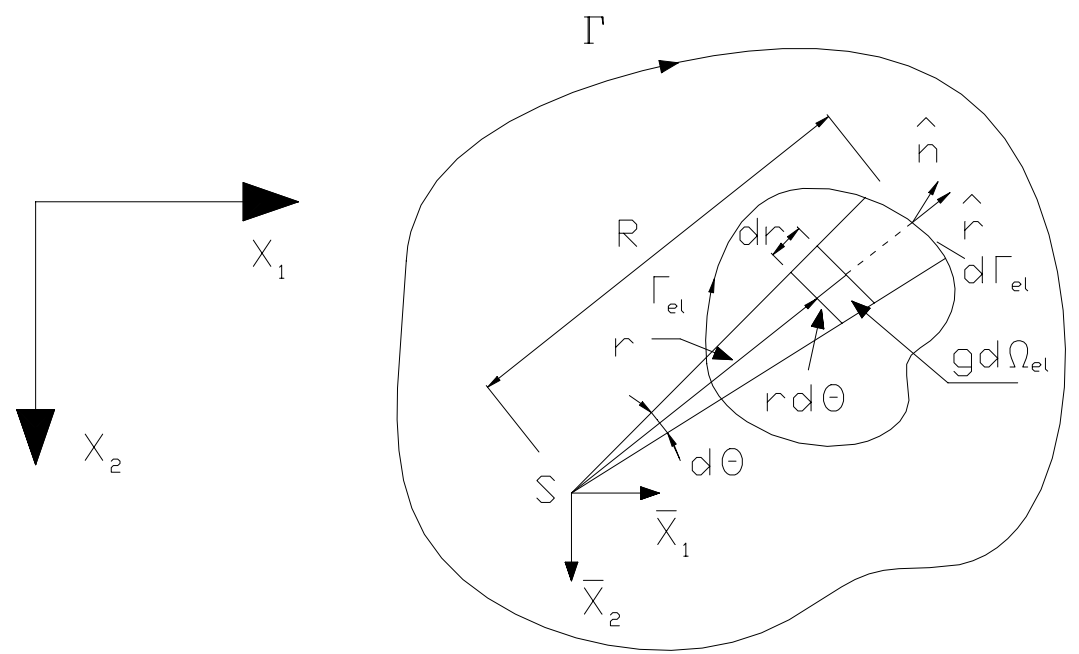

Figura 4.4 - Integração sobre a célula carregada.

Assim:

$\left(\begin{array}{l}\mathrm{x}_{1} \\ \mathrm{x}_{2}\end{array}\right)=\left(\begin{array}{l}\mathrm{x}_{1 \mathrm{~s}} \\ \mathrm{x}_{2 \mathrm{~s}}\end{array}\right)+\left(\begin{array}{l}\mathrm{x}_{1} \\ \mathrm{x}_{2}\end{array}\right)$

As forças de superfície, no sistema $\left(\mathrm{x}_{1}, \mathrm{x}_{2}\right)$ podem ser escrita como: 
$p_{i}=A_{i} \cdot x_{1}+B_{i} \cdot x_{2}+D$

onde:

$D_{i}=A_{i} \cdot x_{1 S}+B_{i} \cdot x_{2 S}+C$

Torna-se necessário integrar as soluções fundamentais $\mathrm{u}_{\mathrm{ij}}{ }^{*}$ no domínio do elemento de contorno. PAIVA (1993), resolve estas integrais de domínio $\Omega_{\mathrm{EL}}$, transformando-as em integrais semi - analítica equivalentes, que requer apenas integração ao longo do contorno do elemento $\Gamma_{\mathrm{EL}}$, isto é, ao longo dos lados do triângulo. $\mathrm{O}$ método consiste em transformar o sistema cartesiano local em coordenadas polares $(r, \theta)$. Dessa forma as forças de superfície podem ser escritas como:

$p_{i}=A_{i} \cdot r \cos \theta+B_{i} \cdot r \operatorname{sen} \theta+D$

$\mathrm{d} \Omega_{\mathrm{EL}}=\mathrm{rdr} \mathrm{d} \theta$

Como exemplo, fazendo apenas a parcela referente a solução fundamental $u_{33}$ na direção de $\mathrm{p}_{\mathrm{i}}$ :

$\int_{\theta}^{\cdot} \int_{0}^{\mathrm{R}_{0}}(\mathrm{~A} \cdot \mathrm{R} \cdot \cos \theta+\mathrm{B} \cdot \mathrm{R} \cdot \operatorname{sen} \theta+\mathrm{D}) \cdot \mathrm{u}_{33} \cdot \mathrm{R} d \mathrm{R} d \theta$

Substituindo $\mathrm{u}_{33}$ na integral:

$\int_{\theta}^{\cdot} \int_{0}^{\mathrm{R}_{0}} \frac{1-\mathrm{v}^{2}}{\pi \cdot \mathrm{E} \cdot \mathrm{R}}\left(\mathrm{A}_{\mathrm{i}} \cdot \mathrm{R} \cdot \cos \theta+\mathrm{B}_{\mathrm{i}} \cdot \mathrm{R} \cdot \operatorname{sen} \theta+\mathrm{D}_{\mathrm{i}}\right) \cdot \mathrm{R} d \mathrm{R} d \theta$

Integrando analiticamente ao longo do raio vetor $\mathrm{r}$ : 
$\int_{\theta}^{\cdot} \frac{1-v^{2}}{2 \pi \cdot E}\left[A_{i} \cdot\left(R_{0}\right)^{2} \cdot \cos \theta+B_{i} \cdot\left(R_{0}\right)^{2} \cdot \operatorname{sen} \theta+2 \cdot D_{i} \cdot R_{0}\right] d \theta$

E o diferencial $\mathrm{d} \theta$ pode ser escrito em função do diferencial de contorno do elemento $\mathrm{d} \Gamma_{\mathrm{EL}}$ :

$\mathrm{d} \theta=\frac{\mathrm{n} \times \mathrm{r}}{\mathrm{R}_{0}} \cdot \mathrm{d} \Gamma_{\mathrm{el}}$

Fazendo a substituição, temos

$\int_{\Gamma_{\mathrm{el}}}^{\cdot} \frac{1-v^{2}}{2 \pi \cdot E}\left(A_{i} \cdot R_{0} \cdot \cos \theta+B_{i} \cdot R_{0} \cdot \operatorname{sen} \theta+2 \cdot D_{i}\right) \cdot n \times r d \Gamma_{e l}$

Agora basta integrar a equação restante ao longo dos lados do elemento. Essa segunda parte da integral é resolvida numericamente, utilizando a regra da quadratura de Gauss.

As demais parcelas das integrais, referentes às soluções fundamentais nas outras direções são dadas abaixo:

$\begin{array}{ll}\text { Para } \mathrm{u}_{11}: & \int_{\Gamma_{\mathrm{el}}}^{\cdot} \frac{1+\mathrm{v}}{2 \pi \cdot \mathrm{E}}\left\lfloor 1-\mathrm{v}+\mathrm{v} \cdot(\cos \theta)^{2}\right\rfloor \cdot\left(\mathrm{A}_{\mathrm{i}} \cdot \mathrm{R}_{0} \cdot \cos \theta+\mathrm{B}_{\mathrm{i}} \cdot \mathrm{R}_{0} \cdot \operatorname{sen} \theta+2 \cdot \mathrm{D}_{\mathrm{i}}\right) \cdot \mathrm{n} \times \mathrm{rd} \Gamma_{\mathrm{el}} \\ \text { Para } \mathrm{u}_{12}: & \int_{\Gamma_{\mathrm{el}}}^{\cdot} \frac{(1+\mathrm{v}) \cdot \mathrm{v} \cdot \cos \theta \cdot \operatorname{sen} \theta}{2 \pi \cdot \mathrm{E}}\left(\mathrm{A}_{\mathrm{i}} \cdot \mathrm{R}_{0} \cdot \cos \theta+\mathrm{B}_{\mathrm{i}} \cdot \mathrm{R}_{0} \cdot \operatorname{sen} \theta+2 \cdot \mathrm{D}_{\mathrm{i}}\right) \cdot \mathrm{n} \times \mathrm{rd} \Gamma_{\mathrm{el}} \\ \text { Para } \mathrm{u}_{13}: & \int_{\Gamma_{\mathrm{el}}}^{\cdot} \frac{(1+\mathrm{v}) \cdot(1-2 \cdot \mathrm{v}) \cdot \cos \theta}{4 \pi \cdot \mathrm{E}}\left(\mathrm{A}_{\mathrm{i}} \cdot \mathrm{R}_{0} \cdot \cos \theta+\mathrm{B}_{\mathrm{i}} \cdot \mathrm{R}_{0} \cdot \operatorname{sen} \theta+2 \cdot \mathrm{D}_{\mathrm{i}}\right) \cdot \mathrm{n} \times \mathrm{rd} \Gamma_{\mathrm{el}}\end{array}$ 


$$
\begin{array}{cl}
\text { Para } \mathrm{u}_{21}: & \int_{\Gamma_{\mathrm{el}}}^{\cdot} \frac{(1+\mathrm{v}) \cdot \mathrm{v} \cdot \cos \theta \cdot \operatorname{sen} \theta}{2 \pi \cdot \mathrm{E}}\left(\mathrm{A}_{\mathrm{i}} \cdot \mathrm{R}_{0} \cdot \cos \theta+\mathrm{B}_{\mathrm{i}} \cdot \mathrm{R}_{0} \cdot \operatorname{sen} \theta+2 \cdot \mathrm{D}_{\mathrm{i}}\right) \cdot \mathrm{n} \times \mathrm{rd} \Gamma_{\mathrm{el}} \\
\text { Para } \mathrm{u}_{22}: & \int_{\Gamma_{\mathrm{el}}}^{\cdot} \frac{1+\mathrm{v}}{\pi \cdot \mathrm{E}}\left[1-\mathrm{v}+\mathrm{v} \cdot(\operatorname{sen} \theta)^{2}\right] \cdot\left(\mathrm{A}_{\mathrm{i}} \cdot \mathrm{R}_{0} \cdot \cos \theta+\mathrm{B}_{\mathrm{i}} \cdot \mathrm{R}_{0} \cdot \operatorname{sen} \theta+2 \cdot \mathrm{D}_{\mathrm{i}}\right) \cdot \mathrm{n} \times \mathrm{rd} \Gamma_{\mathrm{el}} \\
\text { Para } \mathrm{u}_{23}: & \int_{\Gamma_{\mathrm{el}}}^{\cdot} \frac{(1+\mathrm{v}) \cdot(1-2 \cdot \mathrm{v}) \cdot \operatorname{sen} \theta}{4 \pi \cdot \mathrm{E}}\left(\mathrm{A}_{\mathrm{i}} \cdot \mathrm{R}_{0} \cdot \cos \theta+\mathrm{B}_{\mathrm{i}} \cdot \mathrm{R}_{0} \cdot \operatorname{sen} \theta+2 \cdot \mathrm{D}_{\mathrm{i}}\right) \cdot \mathrm{n} \times \mathrm{rd} \Gamma_{\mathrm{el}} \\
\text { Para } \mathrm{u}_{31}: & \int_{\Gamma_{\mathrm{el}}}^{\cdot} \frac{-(1+\mathrm{v}) \cdot(1-2 \cdot \mathrm{v}) \cdot \cos \theta}{4 \pi \cdot \mathrm{E}}\left(\mathrm{A}_{\mathrm{i}} \cdot \mathrm{R}_{0} \cdot \cos \theta+\mathrm{B}_{\mathrm{i}} \cdot \mathrm{R}_{0} \cdot \operatorname{sen} \theta+2 \cdot \mathrm{D}_{\mathrm{i}}\right) \cdot \mathrm{n} \times \mathrm{rd} \Gamma_{\mathrm{el}} \\
\text { Para } \mathrm{u}_{32}: & \int_{\Gamma_{\mathrm{el}}}^{\cdot} \frac{-(1+\mathrm{v}) \cdot(1-2 \cdot \mathrm{v}) \cdot \operatorname{sen} \theta}{4 \pi \cdot \mathrm{E}}\left(\mathrm{A}_{\mathrm{i}} \cdot \mathrm{R}_{0} \cdot \cos \theta+\mathrm{B}_{\mathrm{i}} \cdot \mathrm{R}_{0} \cdot \operatorname{sen} \theta+2 \cdot \mathrm{D}_{\mathrm{i}}\right) \cdot \mathrm{n} \times \mathrm{rd} \Gamma_{\mathrm{el}}
\end{array}
$$

Após efetuar o cálculo das integrais indicadas, obtém-se a representação algébrica do solo, que é dada por:

$\mathrm{H}_{\mathrm{S}} \mathrm{U}_{\mathrm{S}}=\mathrm{G}_{\mathrm{S}} \mathrm{P}_{\mathrm{S}}$

onde:

$\mathrm{P}_{\mathrm{S}}$ : Vetor que contêm as forças de superfície dos nos dos elementos de contorno,

$\mathrm{U}_{\mathrm{S}}$ : Vetor que contêm os deslocamentos dos nos dos elementos de contorno,

$\mathrm{G}_{\mathrm{S}} \quad$ Matriz que contêm os coeficientes da influência do solo.

Para o caso em que a malha discretiza parte da superfície do semi-espaço, a matriz $\mathrm{H}_{\mathrm{S}}$ torna-se igual a matriz identidade. Dessa forma, temos na forma matricial:

$\mathrm{U}_{\mathrm{S}}=\mathrm{G}_{\mathrm{S}} \mathrm{P}_{\mathrm{S}}$ 


\subsection{ACOPLAMENTO MEC/MEF}

Nos itens anteriores, foram apresentados os sistemas algébricos finais, a partir do método dos elementos finitos e do método dos elementos de contorno. Para que o acoplamento entre os sistemas algébricos oriundos do MEC e do MEF seja possível, é necessário a consideração das condições de equilíbrio e compatibilidade existentes nos pontos nodais comuns aos dois métodos.

De forma simplificada, o acoplamento dessas duas técnicas numéricas consiste em transformar a região discretizada em elementos de contorno num elemento finito equivalente, ou vice versa. Nesse trabalho, optou-se pela primeira alternativa, já que o objetivo desse trabalho é analisar a influencia do solo no comportamento das estruturas.

Porém sabe-se que a representação das forças externas no sistema algébrico do MEC é tomada como força por unidade de área, enquanto que nos MEF, é empregado o conceito de equivalente nodal. Assim torna-se necessário adotar um campo de forças comum nos dois sistemas algébricos.

Seja uma célula com um carregamento transversal distribuído por unidade de área.como mostra a figura abaixo: 

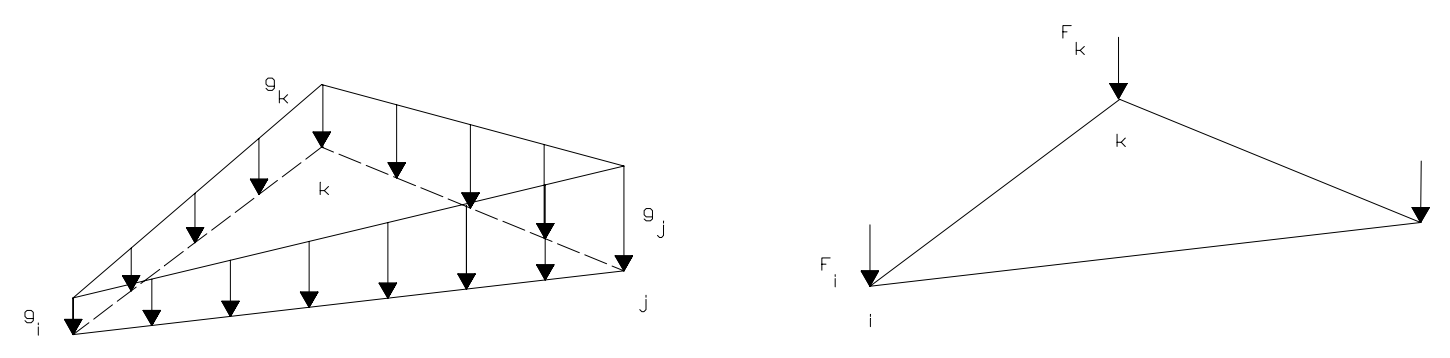

Figura 4.5 - Forças de superfície e carga nodal equivalente.

O trabalho das cargas externas pode ser expresso por:

$$
\mathrm{T}_{\mathrm{e}}=\int_{\mathrm{A}} \mathrm{g}\left(\mathrm{x}_{1}, \mathrm{x}_{2}\right) \cdot \mathrm{w}\left(\mathrm{x}_{1}, \mathrm{x}_{2}\right) \mathrm{dA}
$$

Onde $\mathrm{w}\left(\mathrm{x}_{1}, \mathrm{x}_{2}\right)$ são os deslocamentos transversais no domínio do elemento. A é a area do elemento. Para o caso em que este campo possui variação linear, tem-se:

$$
\mathrm{wi}=\mathrm{w}_{\mathrm{i}} \xi_{1}+\mathrm{w}_{\mathrm{j}} \xi_{2}+\mathrm{w}_{\mathrm{k}} \xi_{3}
$$

E analogamente, as forças de superfície podem ser expressas por:

$$
\mathrm{gi}=\mathrm{g}_{\mathrm{i}} \xi_{1}+\mathrm{g}_{\mathrm{j}} \xi_{2}+\mathrm{g}_{\mathrm{k}} \xi_{3}
$$

Transformando-se as coordenadas dos eixos cartesianos para as coordenadas homogêneas e fazendo a substituição das duas ultimas equações: 
$\mathrm{T}_{\mathrm{e}}=\int_{\mathrm{A}}\left(\mathrm{g}_{\mathrm{i}} \cdot \xi_{1}+\mathrm{g}_{\mathrm{j}} \cdot \xi_{2}+\mathrm{gk}_{\mathrm{k}} \cdot \xi_{3}\right) \cdot\left(\mathrm{w}_{\mathrm{i}} \cdot \xi_{1}+\mathrm{w}_{\mathrm{j}} \cdot \xi_{2}+\mathrm{w}_{\mathrm{k}} \cdot \xi_{3}\right) \mathrm{dA}$

Minimizando-se a energia potencial devida às cargas externas e sabendo-se que a integral abaixo

$\int_{A} f\left(\xi_{1}, \xi_{2}, \xi_{3}\right) d A$

Pode ser calculada como:

$\int_{A} f\left(\xi_{1}, \xi_{2}, \xi_{3}\right) d A=2 \cdot A \cdot \frac{\eta_{1} ! \cdot \eta_{2} ! \cdot \eta_{3} !}{\left(\eta_{1}+\eta_{2}+\eta_{3}+2\right) !}$

Chega-se ao vetor de cargas nodais que é dado por:

$$
\left(\begin{array}{c}
F_{i} \\
F_{j} \\
F_{k}
\end{array}\right)=\frac{A}{12}\left(\begin{array}{ccc}
2 & 1 & 1 \\
1 & 2 & 1 \\
1 & 1 & 2
\end{array}\right) \cdot\left(\begin{array}{l}
g_{i} \\
g_{j} \\
g_{k}
\end{array}\right)
$$

Ou seja:

$$
\left(\begin{array}{c}
F_{i} \\
F_{j} \\
F_{k}
\end{array}\right)=|Q| \cdot\left(\begin{array}{l}
g_{i} \\
g_{j} \\
g_{k}
\end{array}\right)
$$

$[Q]=\frac{A}{12}\left(\begin{array}{lll}2 & 1 & 1 \\ 1 & 2 & 1 \\ 1 & 1 & 2\end{array}\right)$ 


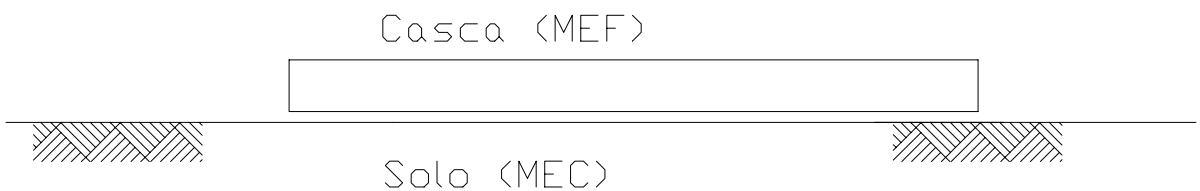

Figura 4.6 - Rede empregada para o solo e a lamina em contato.
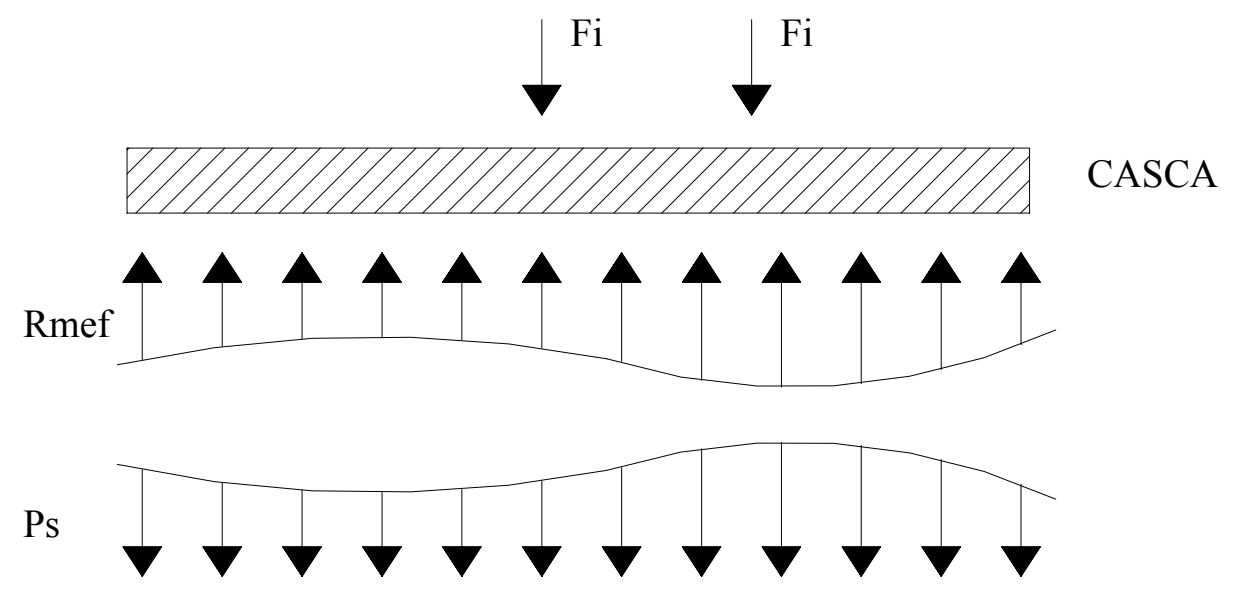

SOLO

Figura 4.7 - Forças de superfície na interface casca-solo

Do sistema algébrico do MEF, conhecida a equação:

$\left[\mathrm{K}_{\mathrm{mef}}\right]_{(6 \mathrm{~N}, 6 \mathrm{~N})} *\left\{\mathrm{U}_{\mathrm{mef}}\right\}_{(6 \mathrm{~N})}=\left\{\mathrm{F}_{\mathrm{mef}}\right\}_{(6 \mathrm{~N})}-\left\{\mathrm{R}_{\mathrm{mef}}\right\}_{(3 \mathrm{~N})}$

onde $\mathrm{N}$ é o número de nós discretizados no solo quanto no casca. 
$\left\{\mathrm{R}_{\text {mef }}\right\}$ pode ser calculado através da seguinte relação:

$\left\{\mathrm{R}_{\mathrm{mef}}\right\}_{(3 \mathrm{~N})}=[\mathrm{Q}]_{(3 \mathrm{~N}, 3 \mathrm{~N})} *\left\{\mathrm{P}_{\mathrm{S}}\right\}_{(3 \mathrm{~N})}$

Lembrando ainda da equação dos deslocamentos do solo, proveniente do MEC:

$\left\{\mathrm{U}_{\mathrm{S}}\right\}_{(3 \mathrm{~N})}=\left\{\mathrm{G}_{\mathrm{S}}\right\}_{(3 \mathrm{~N}, 3 \mathrm{~N})}\left\{\mathrm{P}_{\mathrm{S}}\right\}_{(3 \mathrm{~N})}$

Pode -se ainda escrever:

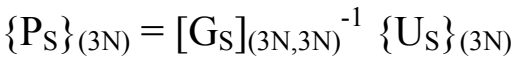

Assim, $\left\{\mathrm{R}_{\mathrm{mef}}\right\}$ pode ser dado por:

$\left\{\mathrm{R}_{\mathrm{mef}}\right\}_{(3 \mathrm{~N})}=[\mathrm{Q}]_{(3 \mathrm{~N}, 3 \mathrm{~N})} *\left[\mathrm{G}_{\mathrm{S}}\right]_{(3 \mathrm{~N}, 3 \mathrm{~N})}{ }^{-1}\left\{\mathrm{U}_{\mathrm{S}}\right\}_{(3 \mathrm{~N})}$

Fazendo agora o equilíbrio na interface solo-casca, temos:

$\left\{\mathrm{U}_{\text {mef }}\right\}=\left\{\mathrm{U}_{\mathrm{S}}\right\}$ e $\{\mathrm{P}\}+\left\{\mathrm{P}_{\mathrm{S}}\right\}=0$

Para que essa igualdade seja verdadeira, é necessário expandir os vetores e as matrizes envolvidas. A expansão é realizada adicionando-se ao vetor ou matriz linhas e/ou colunas nas posições referentes às coordenadas de rotação, existentes na formulação do MEF, mas que não aparecem no MEC.

Assim:

$\left.\left\{\mathrm{R}_{\mathrm{mef}}\right\}_{(6 \mathrm{~N})}=[\mathrm{Q}]_{(6 \mathrm{~N}, 6 \mathrm{~N})} *\left[\mathrm{G}_{\mathrm{S}}\right]_{(6 \mathrm{~N}, 6 \mathrm{~N})}\right)^{-1}\{\mathrm{U}\}_{(6 \mathrm{~N})}$ 
E assim, o novo sistema é dado pela substituição de (4.49) em (4.43):

$\left[\mathrm{K}_{\mathrm{mef}}\right]_{(6 \mathrm{~N}, 6 \mathrm{~N})} *\{\mathrm{U}\}_{(6 \mathrm{~N})}=\left\{\mathrm{F}_{\mathrm{mef}}\right\}_{(6 \mathrm{~N})}-[\mathrm{Q}]_{(6 \mathrm{~N}, 6 \mathrm{~N})} *\left[\mathrm{G}_{\mathrm{S}}\right]_{(6 \mathrm{~N}, 6 \mathrm{~N})}{ }^{-1}\{\mathrm{U}\}_{(6 \mathrm{~N})}$

Juntando os termos em comum, tem-se:

$\left.\left[\mathrm{K}_{\mathrm{mef}}\right]_{(6 \mathrm{~N}, 6 \mathrm{~N})} *\{\mathrm{U}\}_{(6 \mathrm{~N})}+[\mathrm{Q}]_{(6 \mathrm{~N}, 6 \mathrm{~N})} *\left[\mathrm{G}_{\mathrm{S}}\right]_{(6 \mathrm{~N}, 6 \mathrm{~N})}\right)^{-1}\{\mathrm{U}\}_{(6 \mathrm{~N})}=\left\{\mathrm{F}_{\mathrm{mef}}\right\}_{(6 \mathrm{~N})}$

Fazendo:

$\left[\mathrm{K}_{\text {total }}\right]_{(6 \mathrm{~N}, 6 \mathrm{~N})}=\left[\mathrm{K}_{\mathrm{mef}}\right]_{(6 \mathrm{~N}, 6 \mathrm{~N})}+[\mathrm{Q}]_{(6 \mathrm{~N}, 6 \mathrm{~N})} *\left[\mathrm{G}_{\mathrm{S}}\right]_{(6 \mathrm{~N}, 6 \mathrm{~N})}{ }^{-1}$

Pode-se agrupar todo o sistema num outro equivalente, que é dado por:

$\left[\mathrm{K}_{\text {Total }}\right] *\{\mathrm{U}\}=\left\{\mathrm{F}_{\mathrm{mef}}\right\}$

onde:

[ $\left.\mathrm{K}_{\text {Total }}\right]$ Matriz de que incorpora a influencia da casca como também a do solo.

$\{U\}$ Vetor de deslocamentos, composto por todos as deslocabilidades de todos os nós.

$\left\{\mathrm{F}_{\mathrm{mef}}\right\} \quad$ Vetor de forças nodais equivalentes, oriundo do carregamento externo. 


\section{EXEMPLOS}

Nesta etapa, apresentam-se exemplos comparativos entre ensaios realizados por outros autores e os resultados provenientes do código computacional, desenvolvido no decorrer deste trabalho.

\subsection{EXEMPLO 1 - ENSAIO DE WHITAKER \& COOKE}

Em 1966, Whitaker \& Cooke ensaiaram uma estaca vertical de 12,2 m de comprimento e diâmetro de 0,61 m, submetida apenas à um carregamento axial de $1100 \mathrm{kN}$. O módulo de elasticidade do solo e da estaca são $72400 \mathrm{kN} / \mathrm{m}^{2}$ e 2,067 10 ${ }^{7}$ $\mathrm{kN} / \mathrm{m}^{2}$, respectivamente. 


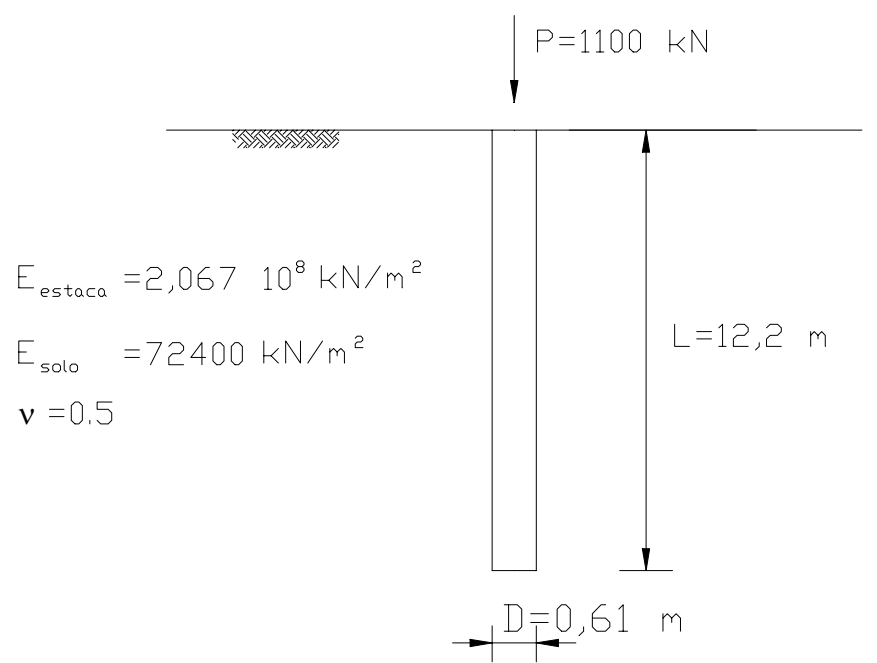

Figura 5.1 - Ensaio de Whitaker \& Cooke

Neste ensaio, Whitaker \& Cooke obtiveram um deslocamento vertical, na cabeça da estaca de 0,284 $\mathrm{cm}$. Utilizando o modelo proposto, obteve-se um deslocamento de $0,289 \mathrm{~cm}$, resultando num erro percentual de $1,8 \%$. Outros autores também analisaram numericamente o ensaio de Whitaker \& Cooke. Ferro obteve como resultado, um deslocamento de $0,2816 \mathrm{~cm}$, discretizando a estaca com apenas 3 nós. Matos Filho obteve para o mesmo problema, um deslocamento de 0,287 cm.

Tal diferença entre os valores obtidos através do modelo proposto em relação ao modelo de Matos Filho, na qual este trabalho foi baseado, deve-se unicamente a parcela referente a singularidade na base da estaca, quando os pontos fonte e campo se encontram na mesma cota. Matos Filho resolveu este problema utilizando uma integral analítica enquanto este modelo foi desenvolvido utilizando uma integral numérica.

Contudo, deve-se ressaltar que o modelo proposto apresenta boa concordância com os modelos apresentados por outros autores. 


\subsection{EXEMPLO 2 - AÇÃO DE FORÇA AXIAL}

Neste exemplo, apresenta-se uma estaca isolada de comprimento L e diâmetro $\mathrm{D}$, inclinada em relação ao eixo vertical de um ângulo $\phi$, submetida apenas à ação de uma força axial.

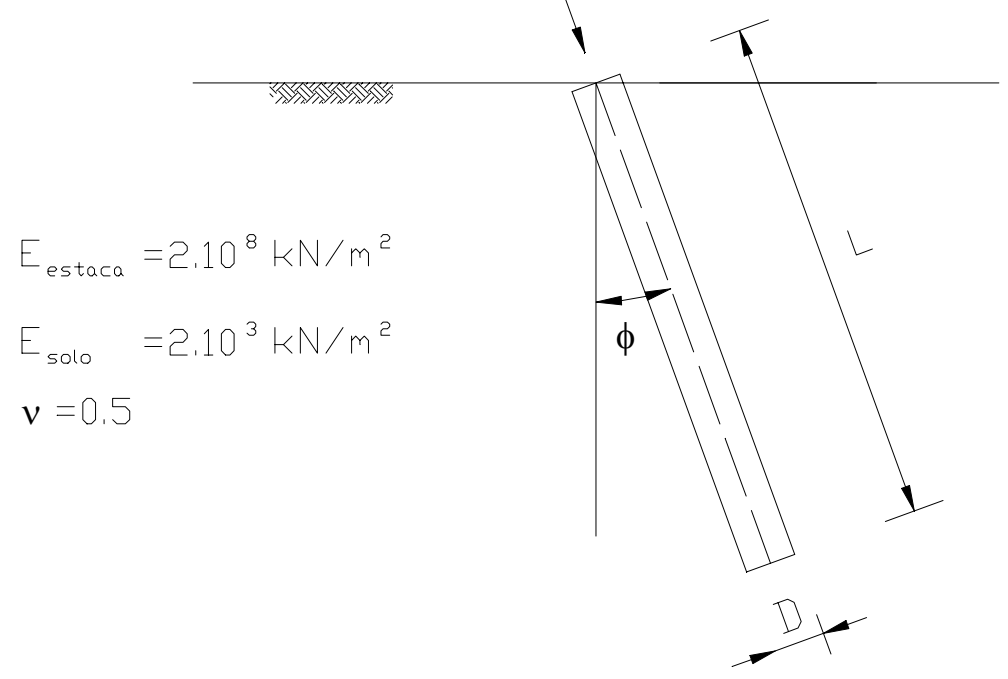

Figura 5.2 - Estaca submetida à ação de uma força axial

Inicialmente fixa-se a inclinação da estaca $\left(\phi=30^{\circ}\right)$ e fazendo uma variação da relação L/D, compara-se os resultados obtidos através desta formulação (programa Pile 2004), com os valores apresentados por Poulos \& Madhav.

Tabela 5.1 - Deslocamentos na cabeça da estaca

\begin{tabular}{|c|c|c|c|c|}
\hline \multirow{2}{*}{ L/D } & \multicolumn{2}{|c|}{ Deslocamento axial (m) } & \multicolumn{2}{c|}{ Deslocamento transversal (m) } \\
\cline { 2 - 5 } & Poulos \& Madhav & Pile 2004 & Poulos \& Madhav & Pile 2004 \\
\hline $\mathbf{1 0}$ & 0,174996 & 0,182090 & 0,000005 & $-0,007931$ \\
\hline $\mathbf{2 5}$ & 0,123997 & 0,126869 & 0,000004 & $-0,004104$ \\
\hline $\mathbf{1 0 0}$ & 0,063499 & 0,064982 & 0,000003 & $-0,000560$ \\
\hline
\end{tabular}

Nota-se claramente nos resultados obtidos por Poulos \& Madhav, que praticamente, forças axiais geram apenas deslocamentos axiais. Comparando os 
resultados entre os dois modelos, nota-se que há grande concordância entre ambos. A maior discrepância ocorre nos deslocamentos normais à estaca. Porém, através de uma análise mais detalhada, nota-se que os valores de deslocamentos normais ao fuste, obtidos através do modelo em estudo, são da ordem de $4 \%$ dos deslocamentos axiais e diminuem quando a relação $\mathrm{L} / \mathrm{D}$ aumenta, tendendo à um valor próximo a zero.

Em seguida, para efeito de comparação, fixa-se agora a relação L/D (L=4 m e $\mathrm{D}=40 \mathrm{~cm})$ e varia-se o ângulo de inclinação $(\phi)$ da estaca. Os resultados obtidos através do programa Pile 2004, são apresentados à seguir:

Tabela 5.2 - Deslocamentos axiais para uma estaca com inclinação $\phi$

\begin{tabular}{|c|c|c|c|c|}
\hline \multirow{2}{*}{$\begin{array}{c}\text { Pto de } \\
\text { colocação }\end{array}$} & \multicolumn{4}{|c|}{ Deslocamento axial (m) } \\
\cline { 2 - 5 } & $\phi=\mathbf{0}^{\mathbf{0}}$ & $\phi=\mathbf{1 0}^{\mathbf{0}}$ & $\phi=\mathbf{2 0}^{\mathbf{0}}$ & $\phi=\mathbf{3 0}^{\mathbf{0}}$ \\
\hline $\mathbf{1}$ & 0,1837951 & 0,1833642 & 0,1825301 & 0,1820949 \\
\hline $\mathbf{2}$ & 0,1837505 & 0,1833195 & 0,1824854 & 0,1820502 \\
\hline $\mathbf{3}$ & 0,1837201 & 0,1832892 & 0,1824551 & 0,1820199 \\
\hline $\mathbf{4}$ & 0,1837075 & 0,1832766 & 0,1824425 & 0,1820075 \\
\hline
\end{tabular}

Observa-se também que os deslocamentos axiais praticamente independem do ângulo de inclinação da estaca, ou seja, os deslocamentos axiais de uma estaca inclinada podem ser estimados, de uma maneira simples, através dos deslocamentos de uma estaca de inclinação zero (estaca vertical).

\subsection{EXEMPLO 3 - AÇAO DE FORÇA TRANSVERSAL}

Agora, apresenta-se uma estaca isolada de comprimento L e diâmetro D, inclinada em relação ao eixo vertical de um ângulo $\phi$, submetida apenas à ação de uma força transversal. 


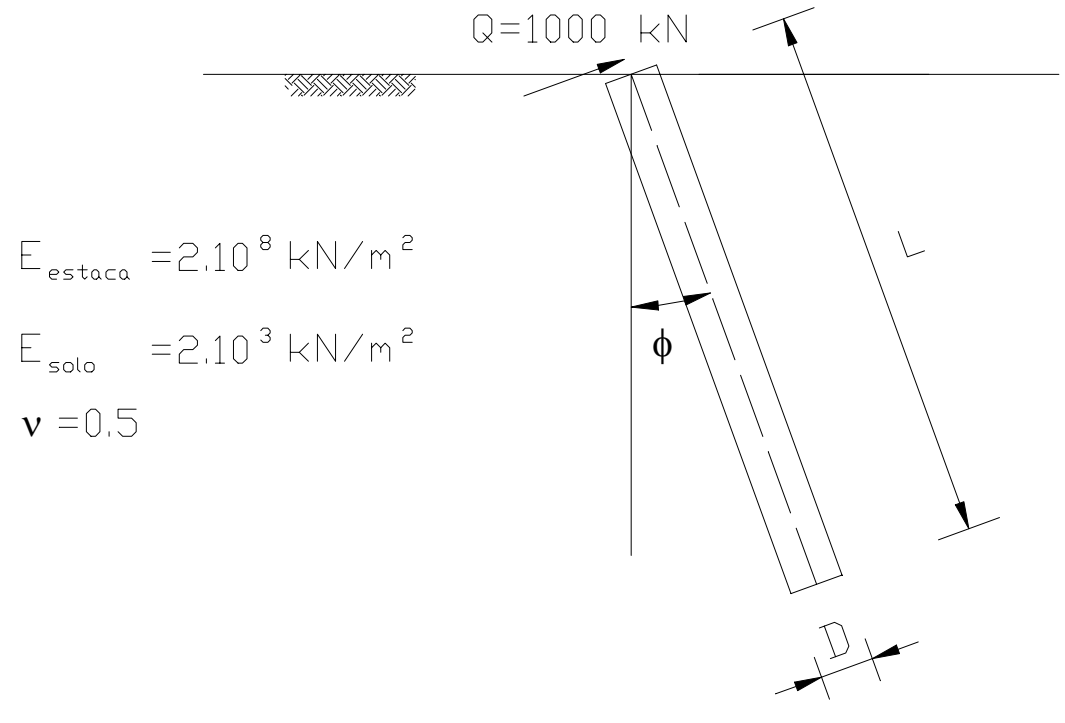

Figura 5.3 - Estaca submetida à ação de uma força transversal.

Da mesma forma que o exemplo anterior fixaremos a inclinação da estaca $\left(\phi=0^{\circ}\right)$ e $\left(\phi=30^{\circ}\right)$ e faremos uma variação da relação L/D, comparando-se os resultados obtidos através desta formulação (programa Pile 2004).

Tabela 5.3 - Deslocamentos na cabeça da estaca

\begin{tabular}{|c|c|c|c|c|}
\hline \multirow{2}{*}{$\mathbf{L} / \mathbf{D}$} & \multicolumn{2}{|c|}{ Deslocamento axial (m) } & \multicolumn{2}{c|}{ Deslocamento transversal (m) } \\
\cline { 2 - 5 } & $\phi=\mathbf{0}^{\mathbf{0}}$ & $\phi=\mathbf{3 0}^{\mathbf{0}}$ & $\phi=\mathbf{0}^{\mathbf{0}}$ & $\phi=\mathbf{3 0}^{\mathbf{0}}$ \\
\hline $\mathbf{1 0}$ & 0,000000 & $-0,004452$ & 0,390540 & 0,399235 \\
\hline $\mathbf{2 5}$ & 0,000000 & $-0,002880$ & 0,307868 & 0,312206 \\
\hline $\mathbf{1 0 0}$ & 0,000000 & $-0,000354$ & 0,340601 & 0,340703 \\
\hline
\end{tabular}

Nota-se claramente, que assim como ocorre com as cargas axiais, forças transversais geram praticamente apenas deslocamentos transversais. Comparando os resultados da estaca vertical e da inclinada, nota-se que há pouca diferença entre os resultados obtidos, podendo-se estimar os deslocamentos da estaca inclinada em função dos resultados obtidos para a estaca vertical. 


\subsection{EXEMPLO 4 - ESTACA INCLINADA}

Neste exemplo, estudaremos o comportamento de uma estaca com um carregamento fixo no seu topo, porém sendo analisada com diferentes ângulos de inclinação.

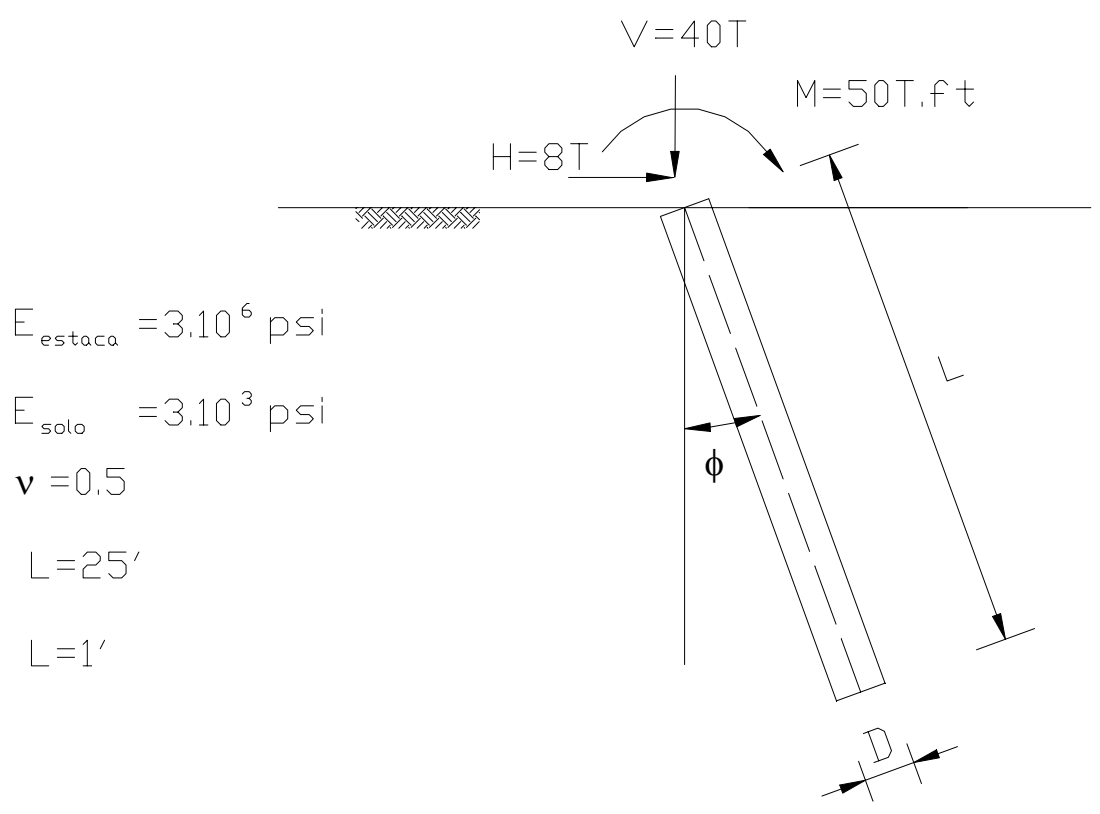

Figura 5.4 - Estaca inclinada sujeita à ação de várias forças.

Os resultados obtidos estão dispostos na forma de tabela, e dois gráficos, representando respectivamente, os deslocamentos na cabeça da estaca (horizontais e verticais), e suas rotações. 
Tabela 5.4 - Deslocamentos na cabeça da estaca

\begin{tabular}{ccccccc}
\hline & & \multicolumn{3}{c}{ Deslocamentos } \\
$\theta$ & $\begin{array}{c}\text { Horizontal (pol) } \\
\text { Poulos \& } \\
\text { Madhav }\end{array}$ & $\begin{array}{c}\text { Pile } \\
\text { Vertical (pol) }\end{array}$ & \multicolumn{2}{c}{$\begin{array}{c}\text { Rotação (rad) } \\
\text { Madhas }\end{array}$} & $\begin{array}{c}\text { Pile } \\
\text { Poulos \& } \\
\text { Madhav }\end{array}$ & $\begin{array}{c}\text { Pile } \\
\end{array}$ \\
& & $\mathbf{2 0 0 4}$ & & $\mathbf{2 0 0 4}$ & & $\mathbf{2 0 0 4}$ \\
\hline $\mathbf{- 3 0}^{\mathbf{0}}$ & 0,70 & 0,62 & 0,56 & 0,54 & 0,0251 & 0,0308 \\
$\mathbf{- 2 0}^{\mathbf{0}}$ & 0,66 & 0,58 & 0,40 & 0,41 & 0,0221 & 0,0275 \\
$\mathbf{- 1 0}^{\mathbf{0}}$ & 0,56 & 0,50 & 0,27 & 0,29 & 0,0185 & 0,0237 \\
$\mathbf{0}^{\mathbf{0}}$ & 0,44 & 0,39 & 0,18 & 0,21 & 0,0152 & 0,0198 \\
$\boldsymbol{+ 1 0}^{\mathbf{0}}$ & 0,30 & 0,27 & 0,16 & 0,17 & 0,0121 & 0,0157 \\
$\boldsymbol{+ 2 0}^{\mathbf{0}}$ & 0,18 & 0,15 & 0,16 & 0,17 & 0,0080 & 0,0116 \\
$\boldsymbol{+ 3 0}^{\mathbf{0}}$ & 0,04 & 0,04 & 0,18 & 0,21 & 0,0050 & 0,0076 \\
\hline
\end{tabular}

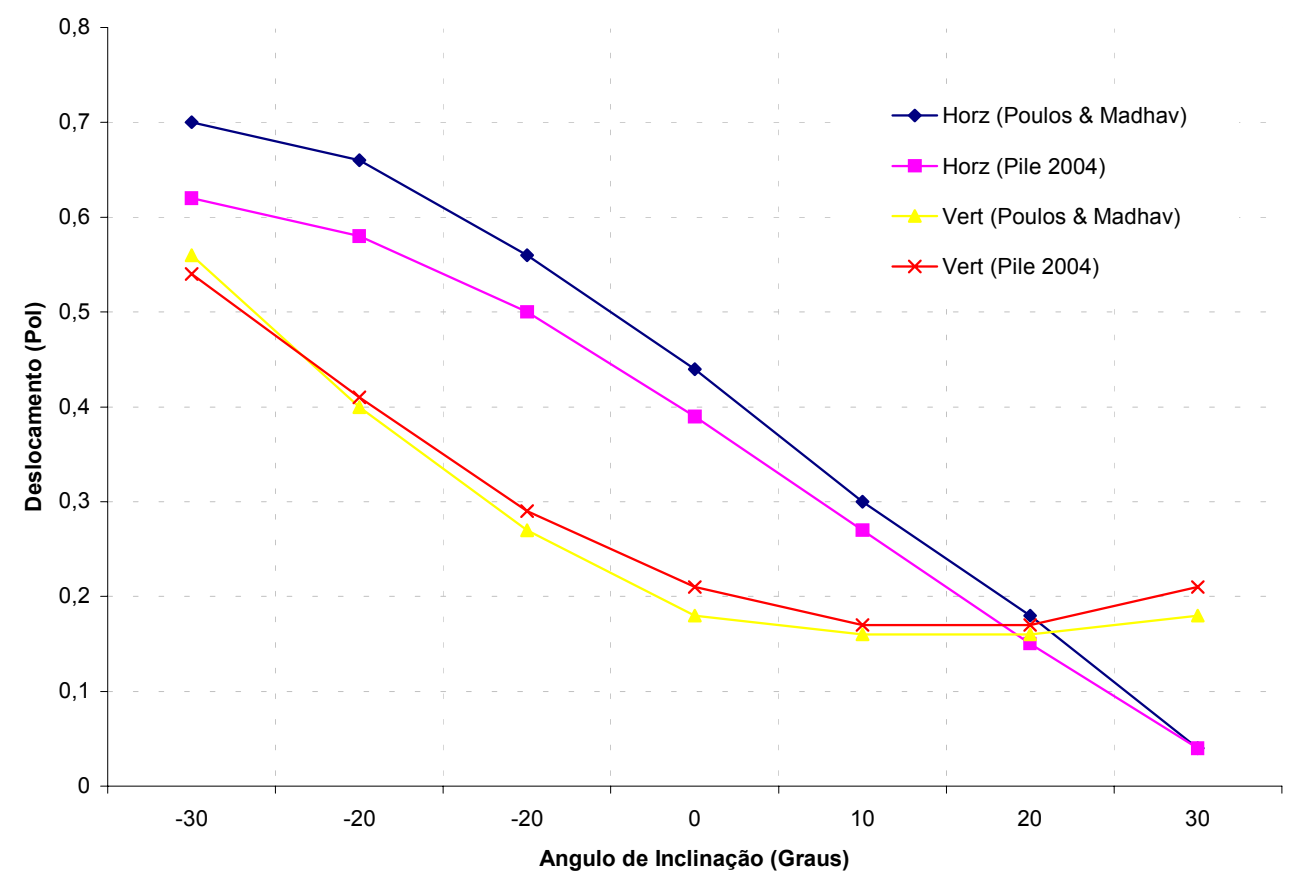

Figura 5.5 - Gráfico deslocamento x Inclinação 


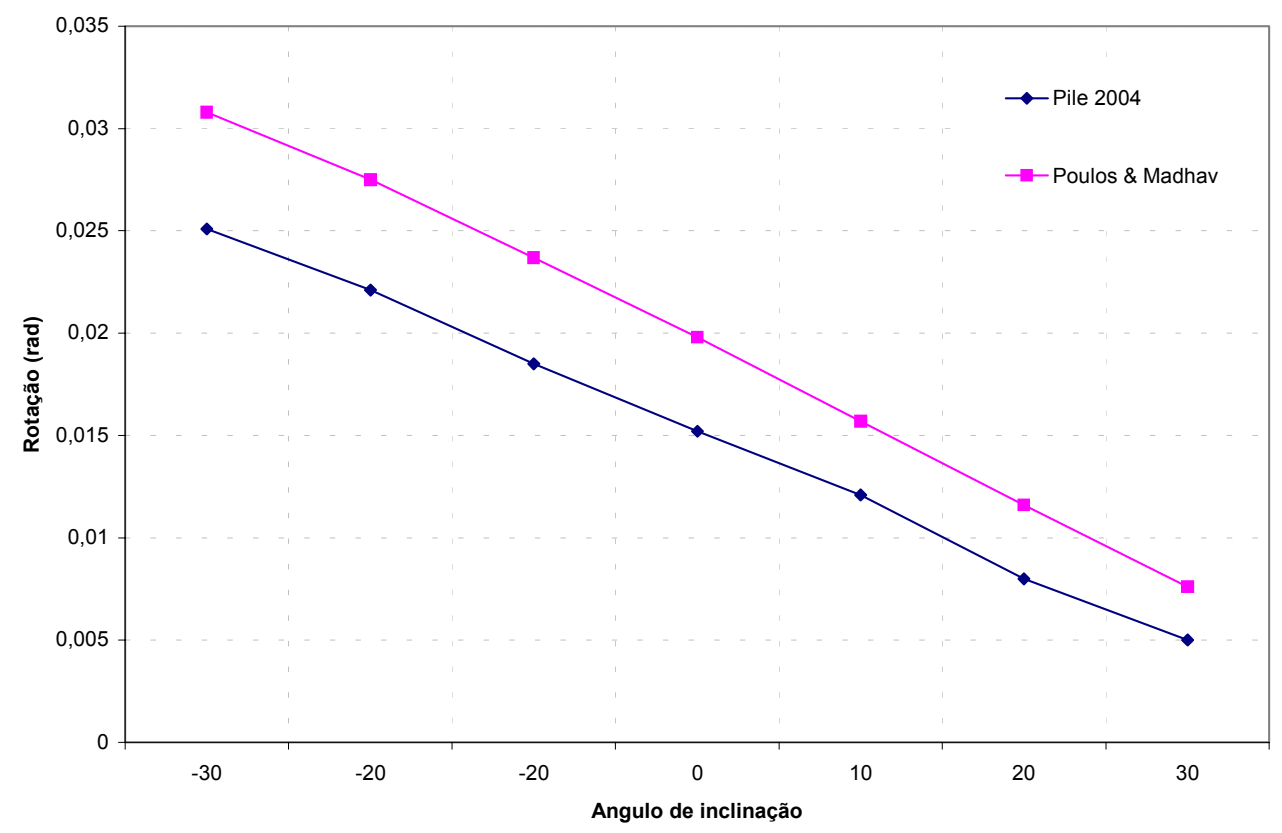

Figura 5.6 - Gráfico rotação x inclinação

Neste exemplo, pode-se observar que os resultados obtidos apresentam boa concordância com aqueles apresentados por Poulos \& Madhav. Nota-se também que os deslocamentos diminuem quando o ângulo de inclinação se aproxima do ângulo na qual se aplica o carregamento.

\subsection{EXEMPLO 5 - GRUPO DE ESTACAS}

Agora, estudaremos um grupo composto por nove estacas idênticas e igualmente espaçadas. As estacas estão divididas em subgrupos, conforme indica a figura abaixo. Em seus respectivos topos serão aplicadas um carregamento horizontal de $20 \mathrm{kN}$. Todas as estacas possuem diâmetro igual a $0,35 \mathrm{~m}$, comprimento de $15 \mathrm{~m}$. O módulo de elasticidade do maciço de solos é $E_{S}=10^{3} \mathrm{kN} / \mathrm{m}^{2}, E_{p}=10^{7} \mathrm{kN} / \mathrm{m}^{2}$. e us $=0,2$. 


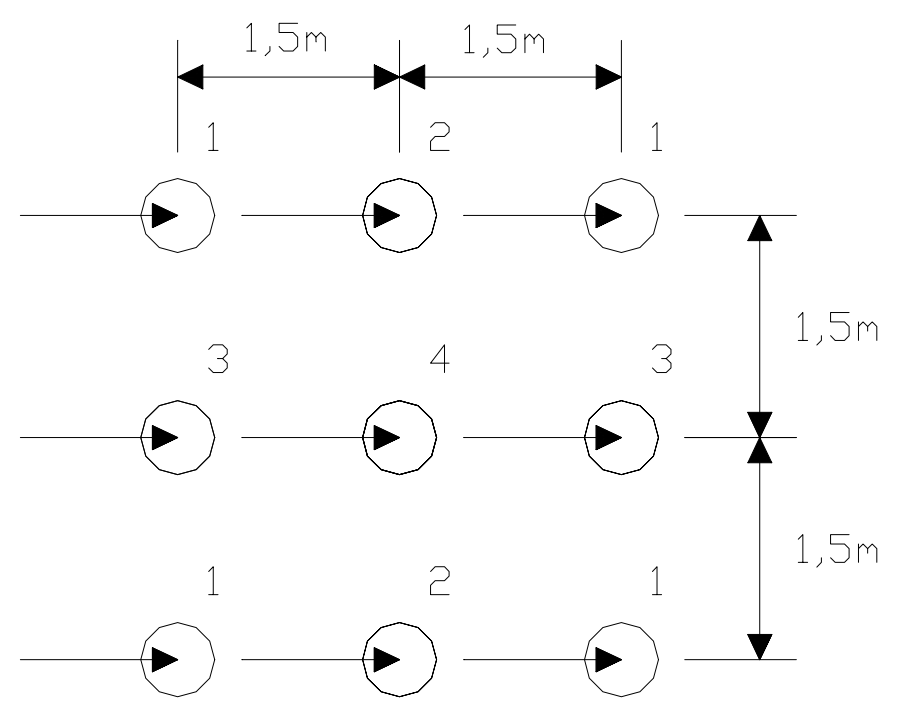

Figura 5.7 - Grupo de estacas

Tabela 5.5 - Deslocamentos horizontais da estaca

\begin{tabular}{|c|c|c|c|c|}
\hline \multirow{2}{*}{ Cotas } & \multicolumn{4}{|c|}{ Deslocamento Horizontal (m) } \\
\cline { 2 - 5 } & Subgrupo 1 & Subgrupo 2 & Subgrupo 3 & Subgrupo 4 \\
\hline 0,00 & 0,0329245 & 0,0352426 & 0,0358183 & 0,0385847 \\
\hline 5,00 & 0,0083437 & 0,0084658 & 0,0087351 & 0,0088904 \\
\hline 10,00 & 0,0040781 & 0,0040573 & 0,0039653 & 0,0039326 \\
\hline 15,00 & 0,0024825 & 0,0024005 & 0,0025326 & 0,0024347 \\
\hline
\end{tabular}

Observando os deslocamentos de cada subgrupo, nota-se claramente que as estacas mais distantes do centro geométrico (subgrupo 1) têm os menores deslocamentos no topo, enquanto as estacas mais ao centro possuem maiores deslocamentos. 


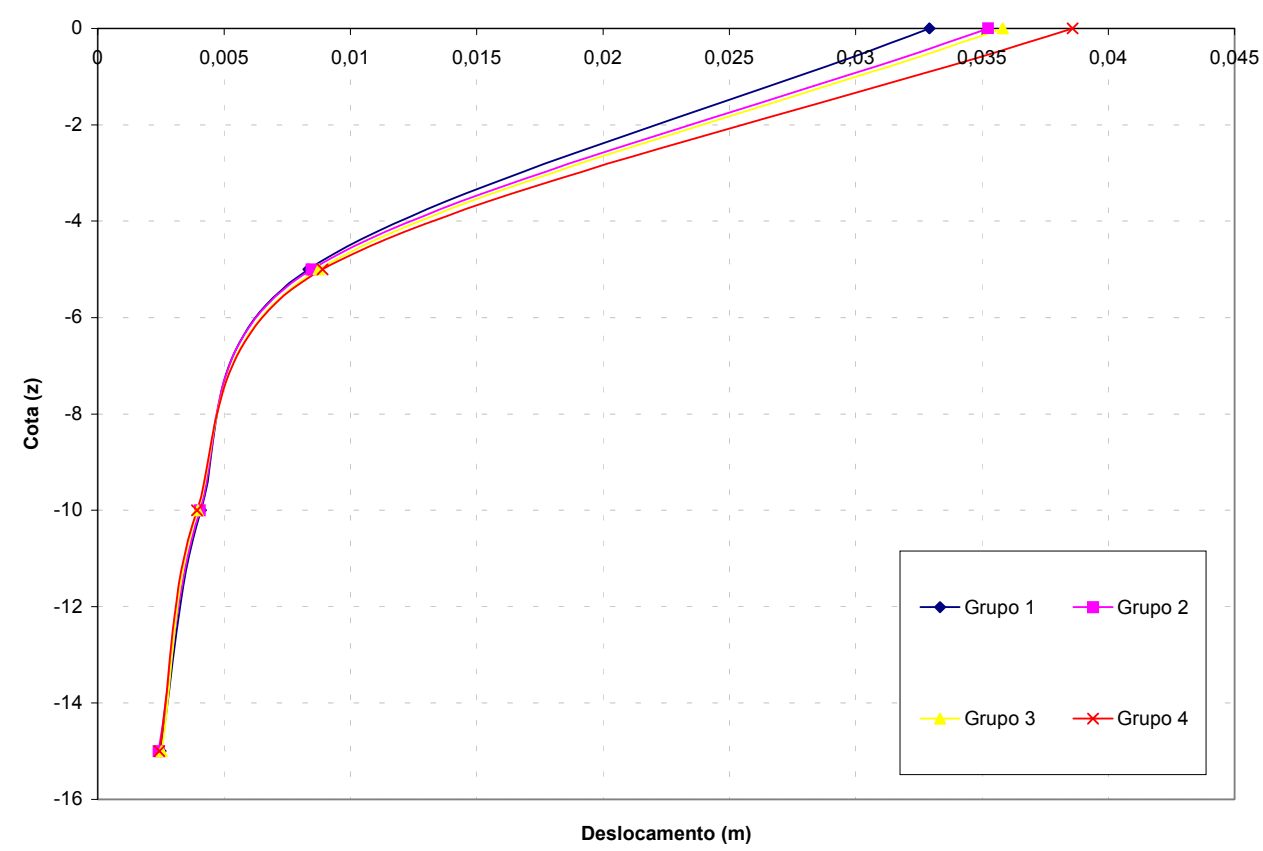

Figura 5.8 - Deslocamento dos grupos de estacas

\subsection{EXEMPLO 6 - BLOCO DE ESTACAS COM CAPEAMENTO RIGIDO}

Este exemplo é um problema retirado de Poulos (1980). Consiste em calcular o deslocamento lateral de um grupo de estacas, todas com diâmetro igual a 1 sob um bloco de capeamento rígido, devido a uma carga de 100 kips na direção $\mathrm{x}_{1}$. Adotouse um coeficiente de Poisson igual a 0,5 e $\mathrm{K}_{\mathrm{r}}=10^{-3}$. $(1 \mathrm{feet}=30,48 \mathrm{~cm}$ e $1 \mathrm{Kip}=$ $4448,22 \mathrm{~N})$ 


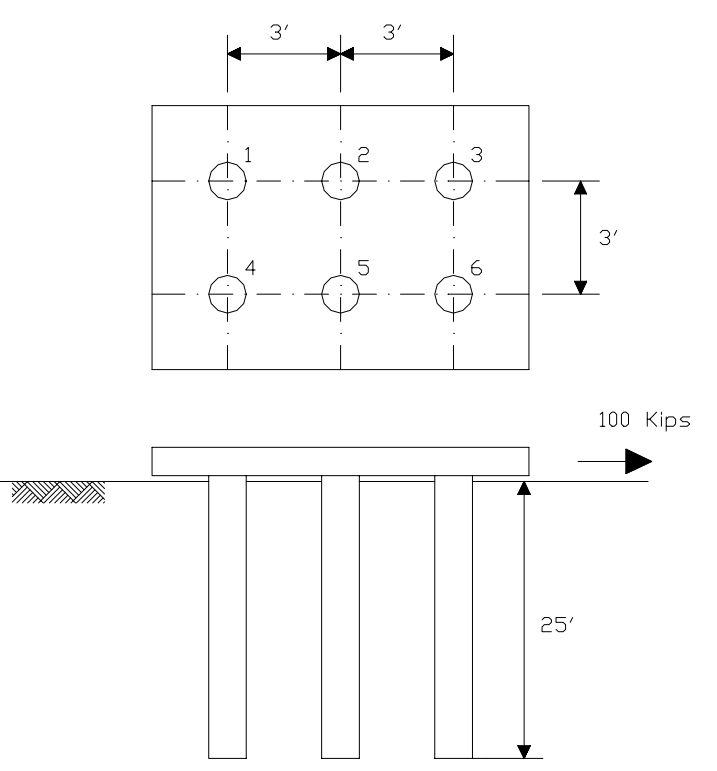

Figura 5.9 - Grupo de estacas com bloco de capeamento rígido.

Esse tipo de problema permite a simulação de estrutura formada por um radier espesso e estaqueado num solo elástico linear, homogêneo, isótropo e semiinfinito. Admite-se que não há contato do bloco com o solo.

A simulação numérica é realizada através da colocação de condições de contorno no topo das estacas, de tal maneira que a cabeça da estaca agora é engastada e todos os elementos deslocam-se igualmente.

Da equação final do acoplamento MEC/MEF, temos que:

$[\mathrm{K}]\{\mathrm{U}\}=\{\mathrm{F}\}$

onde:

[K]: Matriz de rigidez da fundação

$\{\mathrm{U}\}$ : Vetor de deslocamentos laterais e rotações

$\{\mathrm{F}\}$ : Vetor de carregamentos externos no topo da estaca 
Pode-se escrever matricialmente a eq (5.1), da seguinte forma:

$$
\left(\begin{array}{lllll}
\mathrm{k}_{11} & \mathrm{k}_{12} & \mathrm{k}_{13} & \mathrm{k}_{14} & \mathrm{k}_{15} \\
\mathrm{k}_{21} & \mathrm{k}_{22} & \mathrm{k}_{23} & \mathrm{k}_{24} & \mathrm{k}_{25} \\
\mathrm{k}_{31} & \mathrm{k}_{32} & \mathrm{k}_{33} & \mathrm{k}_{34} & \mathrm{k}_{35} \\
\mathrm{k}_{41} & \mathrm{k}_{42} & \mathrm{k}_{43} & \mathrm{k}_{44} & \mathrm{k}_{45} \\
\mathrm{k}_{51} & \mathrm{k}_{52} & \mathrm{k}_{53} & \mathrm{k}_{54} & \mathrm{k}_{55}
\end{array}\right) \cdot\left(\begin{array}{c}
\mathrm{u}_{\mathrm{i}} \\
\theta_{\mathrm{i}} \\
\mathrm{u}_{\mathrm{j}} \\
\mathrm{u}_{\mathrm{k}} \\
\mathrm{u}_{1}
\end{array}\right)=\left(\begin{array}{ccccc}
1 & 0 & 0 & 0 & 0 \\
0 & 1 & 0 & 0 & 0 \\
0 & 0 & 1 & 0 & 0 \\
0 & 0 & 0 & 1 & 0 \\
0 & 0 & 0 & 0 & 1
\end{array}\right) \cdot\left(\begin{array}{c}
\mathrm{F}_{1} \\
\mathrm{M}_{1} \\
0 \\
0 \\
0
\end{array}\right)
$$

Supondo-se agora que foram prescritos o deslocamento e a rotação na cabeça da estaca $\left(u_{i}\right.$ e $\left.\theta_{i}\right)$, nesse caso, faz-se a troca de colunas dos coeficientes de rigidez multiplicáveis pelas suas respectivos valores prescritos no primeiro termo por coeficientes da matriz identidade multiplicáveis pelas suas respectivas forças externas do segundo termo, trocando-se também os sinais.

Dessa maneira, temos:

$$
\begin{aligned}
& \left(\begin{array}{ccccc}
-1 & 0 & \mathrm{k}_{13} & \mathrm{k}_{14} & \mathrm{k}_{15} \\
0 & -1 & \mathrm{k}_{23} & \mathrm{k}_{24} & \mathrm{k}_{25} \\
0 & 0 & \mathrm{k}_{33} & \mathrm{k}_{34} & \mathrm{k}_{35} \\
0 & 0 & \mathrm{k}_{43} & \mathrm{k}_{44} & \mathrm{k}_{45} \\
0 & 0 & \mathrm{k}_{53} & \mathrm{k}_{54} & \mathrm{k}_{55}
\end{array}\right) \cdot\left(\begin{array}{c}
\mathrm{F}_{\mathrm{i}} \\
\mathrm{M}_{\mathrm{i}} \\
\mathrm{u}_{\mathrm{j}} \\
\mathrm{u}_{\mathrm{k}} \\
\mathrm{u}_{1}
\end{array}\right)=\left(\begin{array}{ccccc}
-\mathrm{k}_{11} & -\mathrm{k}_{12} & 0 & 0 & 0 \\
-\mathrm{k}_{21} & -\mathrm{k}_{22} & 0 & 0 & 0 \\
-\mathrm{k}_{31} & -\mathrm{k}_{32} & 1 & 0 & 0 \\
-\mathrm{k}_{41} & -\mathrm{k}_{42} & 0 & 1 & 0 \\
-\mathrm{k}_{51} & -\mathrm{k}_{52} & 0 & 0 & 1
\end{array}\right) \cdot\left(\begin{array}{c}
\mathrm{u}_{\mathrm{i}} \\
\theta_{\mathrm{i}} \\
0 \\
0 \\
0
\end{array}\right) \\
& \text { ou } \\
& {\left[\mathrm{K}^{\prime}\right]^{*}\left\{\mathrm{U}^{\prime}\right\}=\left[\mathrm{I}^{\prime}\right]^{*}\left\{\mathrm{~F}^{\prime}\right\}}
\end{aligned}
$$

onde:

[K']: Matriz que possui coeficientes de rigidez e coeficientes nulos

[I']: Matriz que possui coeficientes de rigidez e coeficientes nulos

$\left\{U^{\prime}\right\}$ : Vetor de forças e deslocamentos incógnitos

$\left\{F^{\prime}\right\}:$ Vetor de forças e deslocamentos prescritos

Multiplica-se a matriz [I'] pelo vetor $\left\{F^{\prime}\right\}$ chegando ao sistema: 
$\left[\mathrm{K}^{\prime}\right]\left\{\mathrm{U}^{\prime}\right\}=\{\mathrm{W}\}$

$\{\mathrm{W}\}=\left[\mathrm{I}^{\prime}\right]^{*}\left\{\mathrm{~F}^{\prime}\right\}$

Resolve-se o sistema (5.5) e obtém se os valores incógnitos tanto de forças, como de deslocamentos.

Este processo é normalmente utilizado para através da prescrição de um deslocamento qualquer para um grupo de estacas, descobrir a carga que nela deverá ser aplicada para que esse grupo desloque igualmente no caso de estacas sob blocos de capeamento rígido.

Dessa forma, para se resolver esse problema, inicialmente assume-se que todas as estacas deslocam igualmente e que sua rotação é restringida.

Prescreve-se um deslocamento unitário e obtêm-se os esforços necessários pra que este deslocamento ocorra. Assim, prescrevendo um deslocamento unitário, tem-se:

Tabela 5.6 - Coeficientes de mola para as estacas

\begin{tabular}{|c|c|c|}
\hline Estacas & $\mathbf{K}_{\mathbf{1}}$ & $\mathbf{K}_{\mathbf{2}}$ \\
\hline $\mathbf{1 , 3}, \mathbf{4}$ e 6 & $10,164 \mathrm{kpis} / \mathrm{pol}$ & \\
\hline $\mathbf{2}$ e 5 & & $7,070 \mathrm{kpis} / \mathrm{pol}$ \\
\hline
\end{tabular}

Obs: $1 \mathrm{Kip} / \mathrm{pol}=1751,27 \mathrm{~N} / \mathrm{cm}, 1 \mathrm{Kip}=4448,22 \mathrm{~N}$ e $1 \mathrm{pol}=2,54 \mathrm{~cm}$

Fazendo o equilíbrio, tem-se:

$4 \mathrm{H} 1+2 \mathrm{H} 2=100$

$\mathrm{K} 1=10,164 \mathrm{kpis} / \mathrm{pol}$

$\mathrm{K} 2=7,070 \mathrm{kpis} / \mathrm{pol}$

$\mathrm{H}_{\mathrm{n}}=\mathrm{K}_{\mathrm{n}} \cdot \mathrm{u}_{\mathrm{n}}$ 


$$
\begin{aligned}
& \mathrm{u}_{1}=\mathrm{u}_{2} \\
& \mathrm{H}_{1}=1,438 \mathrm{H}_{2}
\end{aligned}
$$

Substituindo na equação de equilíbrio, obtêm-se:

$$
\begin{aligned}
& \mathrm{H}_{1}=18,550 \mathrm{kips} \\
& \mathrm{H}_{2}=12,900 \mathrm{kips}
\end{aligned}
$$

Executando mais uma vez esse problema, prescrevendo se como forças na cabeça da estaca, os valores de $\mathrm{H}_{1}$ e $\mathrm{H}_{2}$, obtêm-se:

$$
\mathrm{u} 1=\mathrm{u} 2=1,82 \text { pol. }
$$

E os valores encontrados por Poulos são:

$$
\begin{aligned}
& \mathrm{u}_{\text {POULOS }}=1,53 \text { pol } \\
& \mathrm{H}_{1 \text { POULOS }}=20,1 \mathrm{kips} \\
& \mathrm{H}_{2 \text { POULOS }}=9,8 \mathrm{kips}
\end{aligned}
$$

Observa-se certa discrepância entre os valores encontrados. Porém Poulos estendeu seu método desenvolvido para duas estacas, para análise de grupos genéricos onde todos os espaçamentos deveriam ser idênticos, o que não ocorre nesse exemplo. 


\subsection{EXEMPLO 7 - ESTACAS INCLINADAS}

Este é um exemplo de um grupo de estacas inclinadas. As duas estacas possuem a mesma geometria, as mesmas características físicas e a mesma inclinação. Pretende-se mostrar através deste exemplo que, se o carregamento for simétrico, os deslocamentos das duas estacas logo também serão, independentemente do ângulo de inclinação $\Phi$.

Para esse exemplo, serão consideradas as características das estacas: $\mathrm{L}=10 \mathrm{~m}$, $\mathrm{D}=0,40 \mathrm{~m}$, variando-se o ângulo de inclinação das estacas $\Phi=0^{\circ}$ (caso a - estacas verticais) e $\Phi=30^{\circ}$ (caso b - estacas inclinadas).

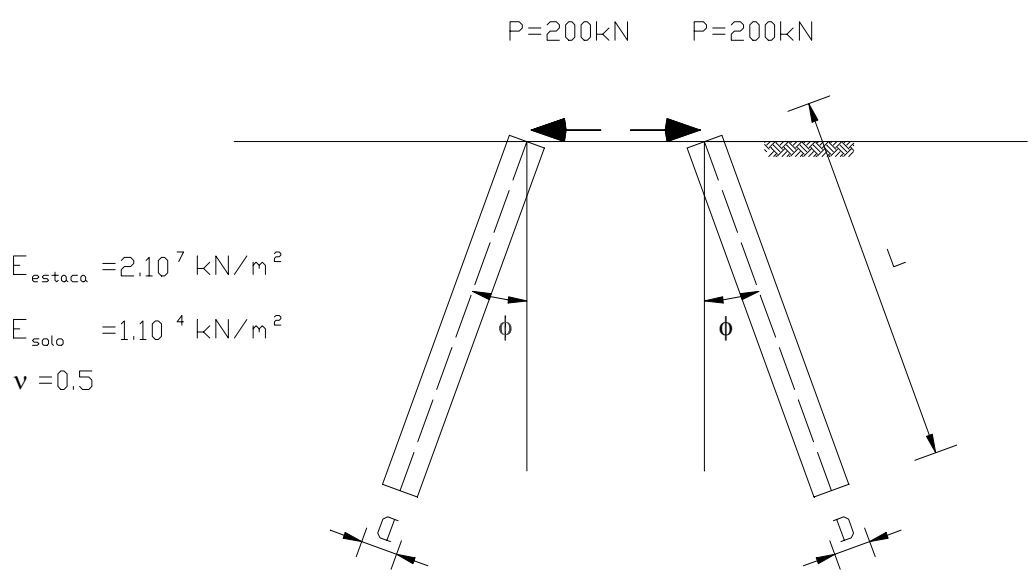

Figura 5.10 - Grupo de estacas inclinadas com simetria 
Tabela 5.7 - Deslocamentos na estaca (caso a - estacas verticais)

\begin{tabular}{|c|c|c|c|c|}
\hline \multirow{2}{*}{$\begin{array}{c}\text { Cotas } \\
(\mathbf{m})\end{array}$} & \multicolumn{2}{|c|}{ Estaca 1 (m) } & \multicolumn{2}{c|}{ Estaca 2 (m) } \\
\cline { 2 - 5 } & Horizontal & Vertical & Horizontal & Vertical \\
\hline 0,00 & $-0,0089701$ & 0,0008295 & 0,0089701 & 0,0008295 \\
\hline 2,89 & 0,0009404 & 0,0008196 & $-0,0009404$ & 0,0008196 \\
\hline 5,77 & $-0,0002492$ & 0,0008418 & 0,0002492 & 0,0008418 \\
\hline 8,66 & 0,0005573 & 0,0008754 & $-0,0005573$ & 0,0008754 \\
\hline
\end{tabular}

Tabela 5.8 - Deslocamentos na estaca (caso b -inclinadas)

\begin{tabular}{|c|c|c|c|c|}
\hline \multirow{2}{*}{$\begin{array}{c}\text { Cotas } \\
(\mathbf{m})\end{array}$} & \multicolumn{2}{|c|}{ Estaca 1 (m) } & \multicolumn{2}{c|}{ Estaca 2 (m) } \\
\cline { 2 - 5 } & Horizontal & Vertical & Horizontal & Vertical \\
\hline 0,00 & $-0,0095484$ & $-0,0021092$ & 0,0095499 & $-0,0021073$ \\
\hline 2,89 & $-0,0000506$ & 0,0032221 & 0,0000518 & 0,0032243 \\
\hline 5,77 & $-0,0008741$ & 0,0026665 & 0,0008753 & 0,0026688 \\
\hline 8,66 & $-0,0008345$ & 0,0026841 & 0,0008358 & 0,0026865 \\
\hline
\end{tabular}

Conforme o esperado, as estacas apresentaram os mesmos deslocamentos, uma vez que ambas possuem as mesmas características geométricas e o mesmo carregamento. 


\subsection{EXEMPLO 8 - ESTACAS INCLINADAS COM CAPEAMENTO RIGIDO}

Este é um exemplo muito interessante de um grupo de estacas inclinadas. As duas estacas possuem a mesma geometria, as mesmas características físicas e a mesma inclinação. As características das estacas são $\mathrm{L}=10 \mathrm{~m}, \mathrm{D}=0,40 \mathrm{~m}$ e $\Phi=30^{\circ}$. Porém em seus topos há um bloco de capeamento rígido. Sob o bloco, uma carga vertical de $200 \mathrm{kN}$.

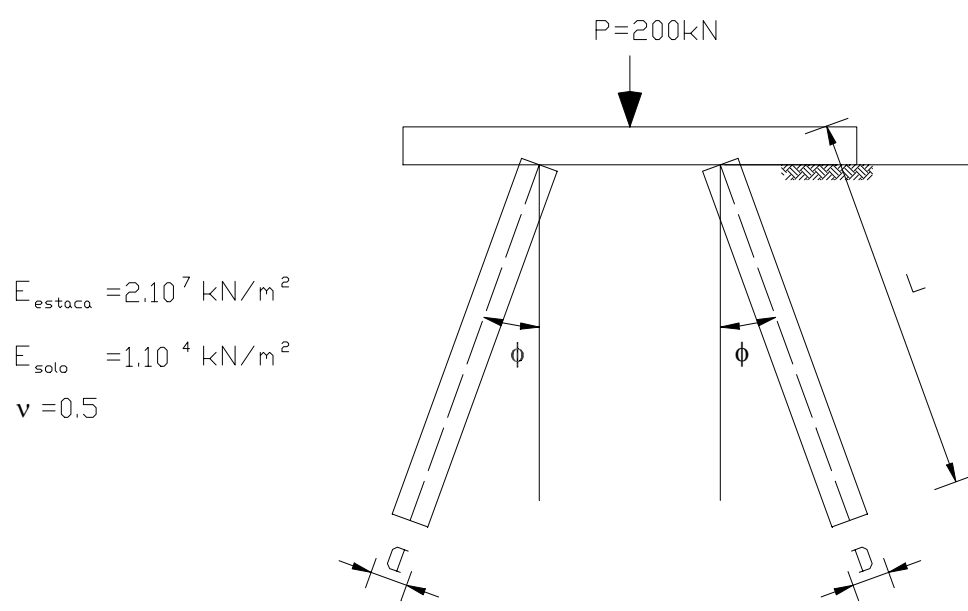

Figura 5.11 - Grupo de estacas inclinadas com bloco de capeamento rígido

Neste exemplo, admite-se agora que quando se aplica a carga concentrada no bloco, as estacas sofrem um deslocamento de uma unidade no sentido do eixo da mesma. Assim, temos para cada estaca: 
Tabela 5.9-Coeficientes de mola para as estacas

\begin{tabular}{|c|c|c|c|c|}
\hline Estacas & $\operatorname{Desc}(\mathbf{x})$ & $\operatorname{Desc}(\mathbf{z})$ & $\mathbf{K}_{\mathbf{1}}(\mathbf{x})$ & $\mathbf{K}_{\mathbf{2}} \mathbf{( z )}$ \\
\hline $\mathbf{1}$ & $+0,5000$ & 0,866 & $-20042,29$ & 32655.57 \\
\hline $\mathbf{2}$ & $-0,5000$ & 0,866 & $+20042,29$ & 32655.57 \\
\hline
\end{tabular}

Sabe-se que $\mathrm{K}_{2}(\mathrm{z})+\mathrm{K}_{2}(\mathrm{z})=200 \mathrm{kN} \quad \therefore \mathrm{K}_{2}(\mathrm{z})=100 \mathrm{kN}$

Portanto, o deslocamento em cada estaca é 3E-3 m. 


\section{CONCLUSÕES}

Neste trabalho foi apresentado uma formulação híbrida do MEC/MEF para avaliar a interação solo-estrutrura.

Comprovou-se que o MEC é um método adequado para se empregar na análise de sólidos tridimensionais de domínio semi-infinito, principalmente por dispensar grandes discretizações do meio infinito ou semi-infinito, reduzindo consideravelmente o numero de variáveis envolvidas.

As estacas foram tratadas como elementos de barra. Através de comparações com modelos de outros autores, constatou-se a boa concordância do modelo apresentado. Os vários exemplos serviram para comprovar a eficiência desta formulação.

No que diz respeito às estacas inclinadas submetidas a um carregamento horizontal ou vertical, notou-se que seu comportamento é muito parecido com a das estacas verticais, pois os deslocamentos são pouco influenciados pelo ângulo de inclinação. Para as estacas inclinadas submetidas à um carregamento qualquer, notase que os deslocamentos tendem a diminuir quanto a inclinação da estaca se aproxima do ângulo na qual se aplica o carregamento. 


\section{BIBLIOGRAFIA}

ALMEIDA, V.S. (2003). Análise da interação solo não homogêneo/estrutura via acoplamento MEC/MEF. São Carlos, 192p. Tese (Doutorado) - Escola de Engenharia de São Carlos - Universidade de São Paulo.

BATOZ, J.L.;BATHE, K.J.; HO, L.W.(1980). A study of three-node triangular plate bending elements. International Journal for Numerical Methods In Engineering, vol 15, p.1771-1812.

BERGAN, P.G. \& FELIPPA, C.A. (1985). A triangular membrane element with rotational degrees of freedom. Comp. Meths. In Appl. Mech. Eng., vol 50, p.2569.

BOUSSINESQ, J. (1885). Applications des potenciels à L'etude de L'equilibre et du moviment des solides. Gualtier-Villars, Paris.

BREBIA, C.A; DOMINGUEZ, J. (1984). Boundary elements: an introductory course. Southampton, CML Publ..

BROMS, B.B. (1965). .Design of lateral loaded piles Journal of Soil Mechanics and Foundation Division. ASCE, vol 91, nº SM3, Mai, p. 79-99.

CERRUTI, V. (1882). Mem. Fis. Mat.. Acc. Lincei, Roma.

DAVINSON, M.T. \& ROBINSON, K.E. (1965). .Bending and buckling of partially embedded piles Proc. Sisth Int. Conf. On Soil Mech. And Found. Eng.. Montreal, vol 2, p. 243-246.

FERRO, N.C.P. (1993). Uma combinação MEC/MEF para análise de fundações enrijecidas por estacas. São Carlos, 135p. Dissertação (Mestrado) - Escola de Engenharia de São Carlos - Universidade de São Paulo.

MATLOCK, H. \& REESE, L.C.(1956). .Non-dimensional solutions for laterally loaded piles with soil modulus assumed proportional to depth. Proc. Eighth Texas Conference on Soil Mechanics and Foundation Engineering. Austin. University of Texas. 
MATLOCK, H. \& REESE, L.C.(1961). .Foundation analysis of offshore pile supported structures. Proc. Fifth Int. Conf. On Soil. Mech. And Found Eng.. Paris, vol.2, p. 91-97.

MATOS FILHO, R.F. (1999). Análise da interação estaca-solo via combinação do método dos elementos finitos com o método dos elementos de contorno. São Carlos, 116p. Dissertação (Mestrado) - Escola de Engenharia de São Carlos Universidade de São Paulo.

MATTES, N.S. \& POULOS, H.G. (1969). Settlement of single compressive pile. Journal of the soil mechanics and foundations division, ASCE, V.97, n.SM1, p.189-207.

MENDONÇA, A.V. (1997). Análise da interação placa-estaca-solo via combinação do método dos elementos finitos com o método dos elementos de contorno. São Carlos, 151p. Dissertação (Mestrado) - Escola de Engenharia de São Carlos Universidade de São Paulo.

MESQUITA, A.D. (1998). Uma formulação do método dos elementos finitos aplicada à análise elastoplástica de cascas. São Carlos, 144p. Dissertação (Mestrado) - Escola de Engenharia de São Carlos - Universidade de São Paulo.

PAIVA, J.B. (1993). Formulação do método dos elementos de contorno para análise da interação solo-estrutura. São Carlos, 183p. Tese (Livre-docência) - Escola de Engenharia de São Carlos - Universidade de São Paulo.

PELETEIRO, S.C. (1996). Utilização da formulação livre para o desenvolvimento de um elemento de membrana com liberdades rotacionais. São Carlos, 101p. Dissertação (Mestrado) - Escola de Engenharia de São Carlos - Universidade de São Paulo.

MINDLIN, R.D. (1936). Force at a point in the interior of a semi-infinite solid. Physics 7 p.195-202, may.

POULOS, H.G. (1968). Analysis of the settlement of pile groups. Geotechnique, vol 18, p. 449-471.

POULOS, H.G. (1971a). Behavior of laterally loaded piles I-Single piles. Journal of the soil mechanics and foundations division, ASCE, V.97, n.SM5, p.711-731.

POULOS, H.G. (1971b). Behavior of laterally loaded piles II-Piles groups. Journal of the soil mechanics and foundations division, ASCE, V.97, n.SM5, p.733-751. 
POULOS, H.G. \& DAVIS, E.H. (1968). The settlement behavior of single axially loaded imcompressible piles and piers. Geotechnique, vol 18, p. 351-371.

POULOS, H.G. \& DAVIS, E.H. (1980). Pile foundation analysis and design. New York, John Wiley \& Sons.

POULOS, H.G. \& MADHAV, M.R. (1971). Analysis of the movement of battered piles. Proc. $1^{\text {st }}$ Aust. N.Z. Conf. On Geomechs., Melbourne, p. 268-275.

SELVADURAI, A.P.S. (1979). Elastic analysis of soil-interaction. Amsterdam. Elsevier Scientific Publishing Company.

SNEDDON, I.N. \& BERRY, D.S. (1958). The classical theory of elasticity. Handuch der Physilk, vol 6, Elasticity and Plasticity. Spring-Verlag, Berlin.

TIMOSHENKO, S.P. \& GOODIER, J.N. (1970). Theory of elasticity. Domicius. New York. McGraw-Hill.

VENTURINI, W.S. (1988). Um estudo sobre o método dos elementos de contorno e suas aplicações em problemas de engenharia. São Carlos. Tese (Livre Docência) - Escola de Engenharia de São Carlos - Universidade de São Paulo.

WATSON, G.N. (1944). A treatise on the theory of Bessel Functions. London. Cambridge University Press.

WINKLER, E. (1867). Die lehre von der Elastizistat und Festigkeit. Domicius. Prage. 